\title{
Completed SDSS-IV extended Baryon Oscillation Spectroscopic Survey: Cosmological implications from two decades of spectroscopic surveys at the Apache Point Observatory
}

Shadab Alam, ${ }^{1}$ Marie Aubert, ${ }^{2}$ Santiago Avila, ${ }^{3,4}$ Christophe Balland, ${ }^{5}$ Julian E. Bautista, ${ }^{6}$ Matthew A. Bershady, ${ }^{7,8,9}$ Dmitry Bizyaev, ${ }^{10,11}$ Michael R. Blanton, ${ }^{12}$ Adam S. Bolton, ${ }^{13,14}$ Jo Bovy, ${ }^{15,16}$ Jonathan Brinkmann, ${ }^{10}$ Joel R. Brownstein, ${ }^{14}$ Etienne Burtin, ${ }^{17}$ Solène Chabanier, ${ }^{17}$ Michael J. Chapman, ${ }^{18,19}$ Peter Doohyun Choi ${ }^{20}$ Chia-Hsun Chuang, ${ }^{21}$ Johan Comparat, ${ }^{22}$ Marie-Claude Cousinou, ${ }^{2}$ Andrei Cuceu, ${ }^{23}$ Kyle S. Dawson $\odot,{ }^{14}$ Sylvain de la Torre, ${ }^{24}$ Arnaud de Mattia,${ }^{17}$ Victoria de Sainte Agathe, ${ }^{5}$ Hélion du Mas des Bourboux ${ }^{14}$ Stephanie Escoffier, ${ }^{2}$ Thomas Etourneau, ${ }^{17}$ James Farr, ${ }^{23}$ Andreu Font-Ribera, ${ }^{25,23}$ Peter M. Frinchaboy, ${ }^{26}$ Sebastien Fromenteau, ${ }^{27}$ Héctor Gil-Marín, ${ }^{28}$ Jean-Marc Le Goff, ${ }^{17}$ Alma X. Gonzalez-Morales, ${ }^{29,30}$ Violeta Gonzalez-Perez, ${ }^{31,6}$ Kathleen Grabowski, ${ }^{10}$ Julien Guy, ${ }^{32}$ Adam J. Hawken, ${ }^{2}$ Jiamin Hou, ${ }^{22}$ Hui Kong, ${ }^{33}$ James Parker III, ${ }^{10}$ Mark Klaene, ${ }^{10}$ Jean-Paul Kneib, ${ }^{34}$ Sicheng Lin, ${ }^{12}$ Daniel Long, ${ }^{10}$ Brad W. Lyke, ${ }^{35}$ Axel de la Macorra, ${ }^{36}$ Paul Martini, $, 37,33$ Karen Masters, ${ }^{38}$ Faizan G. Mohammad, ${ }^{18,19}$ Jeongin Moon, ${ }^{20}$ Eva-Maria Mueller, ${ }^{39}$ Andrea Muñoz-Gutiérrez, ${ }^{36}$ Adam D. Myers, ${ }^{35}$ Seshadri Nadathur, ${ }^{6}$ Richard Neveux, ${ }^{17}$ Jeffrey A. Newman, ${ }^{40}$ Pasquier Noterdaeme ${ }^{41}$ Audrey Oravetz,${ }^{10}$ Daniel Oravetz ${ }^{10}$ Nathalie Palanque-Delabrouille, ${ }^{17}$ Kaike Pan, ${ }^{10}$ Romain Paviot,${ }^{24}$ Will J. Percival, ${ }^{18,19,42}$ Ignasi Pérez-Ràfols, ${ }^{5}$ Patrick Petitjean, ${ }^{41}$ Matthew M. Pieri, ${ }^{24}$ Abhishek Prakash, ${ }^{43}$ Anand Raichoor, ${ }^{34}$ Corentin Ravoux, ${ }^{17}$ Mehdi Rezaie, ${ }^{44}$ James Rich, ${ }^{17}$ Ashley J. Ross, ${ }^{33}$ Graziano Rossi, ${ }^{20}$

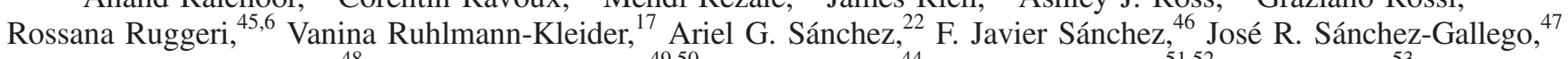
Conor Sayres, ${ }^{48}$ Donald P. Schneider, ${ }^{49,50}$ Hee-Jong Seo, ${ }^{44}$ Arman Shafieloo, ${ }^{51,52}$ Anže Slosar, ${ }^{53}$

Alex Smith, ${ }^{17}$ Julianna Stermer, ${ }^{5}$ Amelie Tamone, ${ }^{34}$ Jeremy L. Tinker, ${ }^{54}$ Rita Tojeiro, ${ }^{55}$ Mariana Vargas-Magaña, ${ }^{36}$ Andrei Variu, ${ }^{34}$ Yuting Wang, ${ }^{56}$ Benjamin A. Weaver, ${ }^{13}$ Anne-Marie Weijmans,${ }^{55}$ Christophe Yèche, ${ }^{17}$ Pauline Zarrouk, ${ }^{57,17}$ Cheng Zhao, ${ }^{34}$ Gong-Bo Zhao, ${ }^{56}$ and Zheng Zheng ${ }^{14}$

${ }^{1}$ University of Edinburgh, Edinburgh EH8 9YL, United Kingdom

${ }^{2}$ Aix Marseille Univ, CNRS/IN2P3, CPPM, Marseille, France

${ }^{3}$ Universidad Autónoma de Madrid, 28049, Madrid, Spain

${ }^{4}$ Instituto de Fisica Teorica UAM/CSIC, Universidad Autonoma de Madrid, 28049 Madrid, Spain

${ }^{5}$ Sorbonne Université, Université Paris Diderot, CNRS/IN2P3,

Laboratoire de Physique Nucléaire et de Hautes Energies, LPNHE, 4 Place Jussieu, F-75252 Paris, France

${ }^{6}$ Institute of Cosmology \& Gravitation, University of Portsmouth, Dennis Sciama Building, Burnaby Road, Portsmouth POI 3FX, United Kingdom

${ }^{7}$ Department of Astronomy, University of Wisconsin-Madison, Madison, Wisconsin 53706, USA

${ }^{8}$ South African Astronomical Observatory, PO Box 9, Observatory 7935, Cape Town, South Africa

${ }^{9}$ Department of Astronomy, University of Cape Town, Private Bag X3, Rondebosch 7701, South Africa

${ }^{10}$ Apache Point Observatory and New Mexico State University, P.O. Box 59, Sunspot, New Mexico 88349, USA

${ }^{11}$ Sternberg Astronomical Institute, Moscow State University, Moscow, 119992, Russia

${ }^{12}$ Center for Cosmology and Particle Physics, Department of Physics, New York University, 726 Broadway, Room 1005, New York, New York 10003, USA

${ }^{13}$ NSF's National Optical-Infrared Astronomy Research Laboratory, 950 N. Cherry Ave, Tucson, Arizona 85719 USA

${ }^{14}$ University of Utah, Department of Physics and Astronomy, 115 S 1400 E, Salt Lake City, Utah 84112, USA

${ }^{15}$ Department of Astronomy and Astrophysics, University of Toronto, Ontario M5S3H4, Canada

${ }^{16}$ Dunlap Institute for Astronomy and Astrophysics, University of Toronto, Ontario M5S 3H4, Canada

${ }^{17}$ IRFU, CEA, Université Paris-Saclay, F-91191 Gif-sur-Yvette, France

${ }^{18}$ Waterloo Centre for Astrophysics, University of Waterloo, Waterloo, Ontario N2L 3G1, Canada

${ }^{19}$ Department of Physics and Astronomy, University of Waterloo,

200 University Ave W, Waterloo, Ontario N2L 3G1, Canada

${ }^{20}$ Department of Physics and Astronomy, Sejong University, Seoul, 143-747, Korea

${ }^{21}$ Kavli Institute for Particle Astrophysics and Cosmology, Stanford 94305, USA

${ }^{22}$ Max-Planck-Institut für extraterrestrische Physik (MPE), Giessenbachstrasse 1, D-85748 Garching bei München, Germany

${ }^{23}$ University College London, WCIE 6BT London, United Kingdom

${ }^{24}$ Aix Marseille Univ, CNRS, CNES, LAM, Marseille, France 
${ }^{25}$ Institut de Física dAltes Energies, The Barcelona Institute of Science and Technology, Campus UAB, 08193 Bellaterra (Barcelona), Spain

${ }^{26}$ Department of Physics \& Astronomy, Texas Christian University, Fort Worth, Texas 76129, USA

${ }^{27}$ ICFUNAM-Instituto de Ciencias Físicas, Universidad Nacional Autónoma de México, 62210 Cuernavaca, Mor., México

${ }^{28}$ ICC, University of Barcelona, IEEC-UB, Martí i Franquès, 1, E08028 Barcelona, Spain

${ }^{29}$ División de Ciencias e Ingenierías, Universidad de Guanajuato, León 37150, México

${ }^{30}$ Consejo Nacional de Ciencia y Tecnología, Av. Insurgentes Sur 1582. Colonia Crédito Constructor, Del. Benito Juárez, C.P. 03940, México D.F. México

${ }^{31}$ Liverpool John Moores University, L3 5RF Liverpool, United Kingdom

${ }^{32}$ Lawrence Berkeley National Laboratory, Berkeley, California 94720, USA

${ }^{33}$ Center for Cosmology and AstroParticle Physics, The Ohio State University, Columbus, Ohio 43212, USA

${ }^{34}$ Institute of Physics, Laboratory of Astrophysics, Ecole Polytechnique Fdrale de Lausanne (EPFL), Observatoire de Sauverny, 1290 Versoix, Switzerland

${ }^{35}$ Department of Physics and Astronomy, University of Wyoming, Laramie, Wyoming 82071, USA

${ }^{36}$ IFUNAM - Instituto de Física, Universidad Nacional Autónoma de México, 04510 CDMX, México

${ }^{37}$ The Ohio State University, Columbus, Ohio 43212, USA

${ }^{38}$ Haverford College, 370 Lancaster Ave, Haverford, Pennsylvania 19041, USA

${ }^{39}$ Sub-department of Astrophysics, Department of Physics, University of Oxford, Denys Wilkinson Building, Keble Road, Oxford OX1 3RH

${ }^{40}$ University of Pittsburgh and PITT PACC, Pittsburgh, Pennsylvania 15260, USA

${ }^{41}$ Institut d'Astrophysique de Paris, CNRS \& Sorbonne University, UMR 7095, 98bis bd Arago, 75014 Paris, France

${ }^{42}$ Perimeter Institute, Waterloo, Ontario N2L 2Y5, Canada

${ }^{43}$ California Institute of Technology, Pasadena, California 91125, USA

${ }^{44}$ Department of Physics and Astronomy, Ohio University, Clippinger Labs, Athens, Ohio 45701, USA

${ }^{45}$ Swinburne University of Technology, Centre for Astrophysics and Supercomputing, Melbourne, VIC 3122, Australia

${ }^{46}$ Fermi National Accelerator Laboratory, Batavia, Illinois 60510, USA

${ }^{47}$ Department of Astronomy, University of Washington, Box 351580, Seattle, Washington 98195, USA

${ }^{48}$ University of Washington, Seattle, Washington 98195, USA

${ }^{49}$ The Pennsylvania State University, University Park, Pennsylvania 16802, USA

${ }^{50}$ Institute for Gravitation and the Cosmos, The Pennsylvania State University, University Park, Pennsylvania 16802, USA

${ }^{51}$ Korea Astronomy and Space Science Institute, Daejeon 34055, Korea

${ }^{52}$ University of Science and Technology, Daejeon 34113, Korea

${ }^{53}$ Brookhaven National Laboratory, Upton, New York 11973, USA

${ }^{54}$ New York University, New York, New York 10003, USA

${ }^{55}$ School of Physics and Astronomy, University of St Andrews, North Haugh, St Andrews, KY16 9SS, United Kingdom

${ }^{56}$ National Astronomical Observatories of China, Chinese Academy of Sciences, 20A Datun Road, Chaoyang District, Beijing 100012, China

${ }^{57}$ Institute for Computational Cosmology, Department of Physics, University of Durham, South Road, Durham DH1 3LE, United Kingdom

(Received 22 July 2020; accepted 16 March 2021; published 28 April 2021)

We present the cosmological implications from final measurements of clustering using galaxies, quasars, and Ly $\alpha$ forests from the completed Sloan Digital Sky Survey (SDSS) lineage of experiments in large-scale structure. These experiments, composed of data from SDSS, SDSS-II, BOSS, and eBOSS, offer independent measurements of baryon acoustic oscillation (BAO) measurements of angular-diameter distances and Hubble distances relative to the sound horizon, $r_{d}$, from eight different samples and six measurements of the growth rate parameter, $f \sigma_{8}$, from redshift-space distortions (RSD). This composite sample is the most constraining of its kind and allows us to perform a comprehensive assessment of the cosmological model after two decades of dedicated spectroscopic observation. We show that the BAO data alone are able to rule out dark-energy-free models at more than eight standard deviations in an extension to the flat, $\Lambda \mathrm{CDM}$ model that allows for curvature. When combined with Planck Cosmic Microwave Background (CMB) measurements of temperature and polarization, under the same model, the BAO data provide nearly an order of magnitude improvement on curvature constraints relative to primary CMB 
constraints alone. Independent of distance measurements, the SDSS RSD data complement weak lensing measurements from the Dark Energy Survey (DES) in demonstrating a preference for a flat $\Lambda$ CDM cosmological model when combined with Planck measurements. The combined BAO and RSD measurements indicate $\sigma_{8}=0.85 \pm 0.03$, implying a growth rate that is consistent with predictions from Planck temperature and polarization data and with General Relativity. When combining the results of SDSS BAO and RSD, Planck, Pantheon Type Ia supernovae (SNe Ia), and DES weak lensing and clustering measurements, all multiple-parameter extensions remain consistent with a $\Lambda \mathrm{CDM}$ model. Regardless of cosmological model, the precision on each of the three parameters, $\Omega_{\Lambda}, H_{0}$, and $\sigma_{8}$, remains at roughly $1 \%$, showing changes of less than $0.6 \%$ in the central values between models. In a model that allows for free curvature and a time-evolving equation of state for dark energy, the combined samples produce a constraint $\Omega_{k}=-0.0022 \pm 0.0022$. The dark energy constraints lead to $w_{0}=-0.909 \pm 0.081$ and $w_{a}=-0.49_{-0.30}^{+0.35}$, corresponding to an equation of state of $w_{p}=-1.018 \pm 0.032$ at a pivot redshift $z_{p}=0.29$ and a Dark Energy Task Force Figure of Merit of 94. The inverse distance ladder measurement under this model yields $H_{0}=68.18 \pm 0.79 \mathrm{~km} \mathrm{~s}^{-1} \mathrm{Mpc}^{-1}$, remaining in tension with several direct determination methods; the BAO data allow Hubble constant estimates that are robust against the assumption of the cosmological model. In addition, the BAO data allow estimates of $H_{0}$ that are independent of the CMB data, with similar central values and precision under a $\Lambda$ CDM model. Our most constraining combination of data gives the upper limit on the sum of neutrino masses at $\sum m_{\nu}<0.115 \mathrm{eV}$ ( $95 \%$ confidence). Finally, we consider the improvements in cosmology constraints over the last decade by comparing our results to a sample representative of the period 2000-2010. We compute the relative gain across the five dimensions spanned by $w, \Omega_{k}, \sum m_{\nu}, H_{0}$, and $\sigma_{8}$ and find that the SDSS BAO and RSD data reduce the total posterior volume by a factor of 40 relative to the previous generation. Adding again the Planck, DES, and Pantheon SN Ia samples leads to an overall contraction in the five-dimensional posterior volume of 3 orders of magnitude.

DOI: 10.1103/PhysRevD.103.083533

\section{INTRODUCTION}

Understanding the energy content of the Universe, the physical mechanisms behind cosmic expansion, and the growth of structure is the primary challenge of cosmology. Developmental milestones for the current standard model of these properties, the spatially flat $\Lambda \mathrm{CDM}$ model, include measurements of the expansion history using Type Ia supernovae (SNe Ia) in the 1990s, which provided the first evidence for cosmic acceleration [1,2], and studies of perturbations in the cosmic microwave background (CMB), which provided the first convincing evidence for a nearly flat geometry [3-6] when assuming weak priors and fitting results from the BOOMERanG [7] and MAXIMA [8] $\mathrm{CMB}$ experiments. These observations showed that prior suggestions that the $\Lambda \mathrm{CDM}$ model would solve some of the emerging problems in cosmology were particularly adroit $[9,10]$. At around the same time as these observations, the first measurements of the baryon and matter densities from the shape of the power spectrum from the $2 \mathrm{dF}$ Galaxy Redshift Survey (2dFGRS) [11] were published [12]. The combination of the galaxy survey data and CMB data is particularly strong for breaking degeneracies inherent to either method individually: combining early 2dFGRS and CMB data meant that, at around the turn of the century, the physical baryon and cold dark matter densities were known to $10 \%$ and $8 \%$, respectively, and the Hubble parameter was known to $7 \%$ within the flat $\Lambda \mathrm{CDM}$ model [13].
The first decade of the 21st century witnessed a strong advancement in the precision with which the parameters of this standard model were known, without demonstrating significant tension with this model. This came through dedicated CMB experiments including ACT [14], SPT [15], and the Wilkinson Microwave Anisotropy Probe (WMAP) [16]. SN Ia observations continued to improve in sample size and analysis methodology [17-25], and direct measurements of the local expansion rate using Cepheid variables and $\mathrm{SNe}$ Ia led to estimates of $H_{0}$ with better than $4 \%$ precision [26,27]. During this same approximate period, the 2dFGRS and Sloan Digital Sky Survey (SDSS) [28] galaxy surveys became sufficiently large to clearly measure the baryon acoustic oscillation (BAO) feature in the clustering of galaxies $[29,30]$ and use this as a robust cosmological probe [31]. Combined, these experiments offered strong evidence supporting the simple six-parameter $\Lambda \mathrm{CDM}$ cosmological model consisting of the baryon density $\left(\Omega_{b}\right)$, dark matter density $\left(\Omega_{c}\right)$, Hubble constant $\left(H_{0}\right)$, amplitude of primordial perturbations $\left(A_{s}\right)$, power-law spectral index of primordial density perturbations $\left(n_{s}\right)$, and reionization optical depth $(\tau)$. The five-year WMAP data [32], combined with the SDSS-II BAO data [31] and the union SN sample [33], led to measurements of the physical baryon and cold dark matter densities to $3 \%$ and of the Hubble parameter to $2 \%$ [34] within the framework of the $\Lambda$ CDM model. 
The last ten years have seen significant advances in cosmology through CMB observations, improved calibration of systematic errors in SNe Ia studies, and large area spectroscopic surveys. Gravitational lensing from the $\mathrm{CMB}$ has provided important, high signal-to-noise measurements of structure growth in the low-redshift universe $[35,36]$. CMB lensing has been supplemented by increasingly robust and statistically sensitive estimates of weak lensing based on galaxy shapes, including CFHTLenS [37], KiDS [38,39], Dark Energy Survey (DES) [40,41], and Hyper Suprime-Cam survey (HSC) [42,43]. Improvements in the statistical precision from weak lensing programs have begun through joint analysis of multiple datasets, using consistent priors, modeling of the power spectrum, and redshift distributions (e.g., Ref. [44]).

While broad support for the flat $\Lambda \mathrm{CDM}$ model remains among measurements, a number of increasingly worrying discrepancies are appearing. For example, measurements of the Hubble constant from the distance ladder technique (SH0ES) [45] and those from the Planck CMB measurements [46] are in tension, as discussed further in Sec. IV B. Additionally, albeit at lower levels of discordance, weak lensing measurements (e.g., Refs. [38,39]) tend to find lower levels of cosmological structure than found from redshift-space distortions or predicted from the amplitude of the Planck CMB power spectrum (see Sec. II C 3).

The continuing development of massive spectroscopic surveys over the last decade is of particular interest to this study. Spectroscopy of galaxies and quasars over wide areas allows precise measurements of cosmic expansion history with $\mathrm{BAO}$ and measurements of the rate of structure growth with redshift-space distortions (RSD). The largest spectroscopic survey to date is the Baryon Oscillation Spectroscopic Survey (BOSS) [47], which was the primary driver for SDSS-III [48]. In operations spanning 2009-2014, BOSS completed spectroscopy on more than 1.5 million galaxies as faint as $i=19.9$ and more than $160,000 z>2.1$ quasars as faint as $g=22$. In 2012, the first BAO measurements from BOSS were published [49], just before the final results from the WMAP CMB experiment. At this point, the data were sufficient to set interesting constraints on models that go beyond $\Lambda \mathrm{CDM}$. For example, an analysis under a flat $\Lambda \mathrm{CDM}$ model with neutrinos using the final WMAP data, an estimate of $H_{0}=73.8 \pm 2.4 \mathrm{~km} \mathrm{~s}^{-1} \mathrm{Mpc}^{-1}$ [50], and the BOSS BAO measurements [49], together with those from the 6dF Galaxy Survey (6dFGS), SDSS-II, and WiggleZ surveys [51-53] led to a 95\% upper limit of $0.44 \mathrm{eV}$ on the sum of the neutrino masses [54]. Adding measurements of luminosity-distance ratios from a large sample of SNe Ia [23-25] led to constraints consistent with a cosmological constant when allowing a Chevallier-PolarskiLinder (CPL) parametrization $[55,56]$ of dark energy, indicating $w_{0}=-1.17_{-0.12}^{+0.13}$ and $w_{a}=0.35_{-0.49}^{+0.50}$ in a model with a flat universe [54]. Within the $\Lambda \mathrm{CDM}$ model, the errors on the physical baryon density and cold dark matter density were now at the level of $2 \%$, and the Hubble constant was at $1.3 \%$.

Final measurements of the CMB-calibrated BAO scale from the BOSS experiment led to $1 \%$ precision measurements of the cosmological distance scale for redshifts $z<$ 0.75 [57] and $2 \%$ precision measurements at $z=2.33$ $[58,59]$. By the time that the final results from BOSS were ready, the Planck satellite had released its 2015 CMB measurements [60], surpassing the precision afforded by WMAP. In combination, the 2015 CMB power spectrum measurements from the Planck satellite together with BOSS constrain the rate of structure growth at the level of $6 \%$ and constrain the sum of the neutrino masses to be less than $160 \mathrm{meV}$ at $95 \%$ confidence [57]. With these data, the constraints on the physical matter density and Hubble constant within the $\Lambda \mathrm{CDM}$ model were both at the level of $0.6 \%$.

So far, there have been four generations of SDSS conducted from the $2.5 \mathrm{~m}$ Sloan Telescope [61] at the Apache Point Observatory. The extended Baryon Oscillation Spectroscopic Survey (eBOSS) $[62,63]$ is the cosmological survey within SDSS-IV [64]. Using the same spectrographs used for BOSS [65], eBOSS concluded 4.5 years of spectroscopic observations of large-scale structure on March 1, 2019. EBOSS extends the BOSS analysis using galaxies as direct tracers of the density field to measure BAO and RSD to higher redshifts and increases the number of quasars used for Ly $\alpha$ forest studies. It also marks the last use of the Sloan Telescope for galaxy redshift surveys designed to measure cosmological parameters using BAO and RSD techniques, with SDSS now focusing on other exciting astronomical questions [66].

In this paper, we characterize the advances made in constraining the cosmological model over the last decade, focusing specifically on the impact of the BOSS and eBOSS spectroscopic programs. A summary of the key results from this work, as well as a few additional figures, can be found in the SDSS webpages [67]. The study presented in this work is part of a coordinated release of the final eBOSS measurements of $\mathrm{BAO}$ and RSD in the clustering of luminous red galaxies (LRGs) [68,69], emission line galaxies (ELGs) [70-72], and quasars [73,74]. At the highest redshifts $(z>2.1)$, the coordinated release of final $\mathrm{eBOSS}$ measurements includes measurements of BAO in the Ly $\alpha$ forest [75]. An essential component of these studies is the construction of data catalogs [76,77], mock catalogs [78-80], and galaxy mocks based on N-body simulations for assessing theoretical systematic errors [81-84]. A summary table of the BAO and RSD measurements, with links to supporting studies and legacy figures describing the measurements, can be found in the SDSS webpages [85].

In all, the SDSS, BOSS, and eBOSS surveys provide galaxy and quasar samples from which $\mathrm{BAO}$ can be measured covering all redshifts $z<2.2$, and $\operatorname{Ly} \alpha$ forest 
observations over $2<z<3.5$. The aggregate precision of the expansion history measurements is $0.70 \%$ at redshifts $z<1$ and $1.19 \%$ at redshifts $z>1$, while the aggregate precision of the growth measurements is $4.77 \%$ over the redshift interval $0<z<1.5$. With this coverage and sensitivity, the SDSS experiment is unparalleled in its ability to explore models of dark energy.

In Sec. II, we present the cosmological background and the signatures in the key observational probes. This section is intended to provide a high-level background that will put the SDSS spectroscopic surveys into the broader context for relatively new readers. In Sec. III, we present the data samples for the cosmological analyses performed in this work. In Sec. IV, we discuss the impact of SDSS BAO distance measurements on single parameter extensions to $\Lambda \mathrm{CDM}$, relative to $\mathrm{SNe}$ Ia and $\mathrm{CMB}$ probes. We also demonstrate the key contributions from BAO measurements in the well-known tension between local measurements of $H_{0}$ and estimates extrapolated from high-redshift observations. In Sec. V, we discuss RSD and weak lensing measurements both in constraining the relative abundance of dark energy and in testing predictions of growth under an assumption of General Relativity (GR). In Sec. VI, we present the cosmological model that best describes all of the observational data used in this work. We conclude in Sec. VII by presenting the substantial advances in our understanding of the cosmological model that have been made in the last decade and the role that the BOSS and eBOSS programs play in those advances.

\section{COSMOLOGICAL MODEL AND OBSERVABLE SIGNATURES}

The BOSS and eBOSS surveys have fostered the development of the BAO technique to percent-level precision over a larger redshift range than any other probe of the distance-redshift relation. RSD measurements from BOSS and eBOSS offer constraints on structure growth over nearly as large a redshift range. Meanwhile, in the last ten years, the CMB maps produced by the Planck satellite have allowed precise constraints on the conditions of the Universe at the time of last scattering and on the angular diameter distance to that epoch. With probes of the latetime expansion history, the evolution of cosmic expansion can be extrapolated from the CMB to today's epoch under models with freedom for curvature, dark energy density, dark energy equation of state, and neutrino mass. SNe Ia measurements remain the most effective way to constrain expansion history at redshifts below $z<0.5$, while the BOSS and eBOSS BAO measurements cover redshifts $0<z<2.5$ and rely on a model that is well understood because it is based in simple physics. Large weak lensing surveys have measured cosmic shear to constrain the local matter density and amplitude of fluctuations, while RSD measure the change in the fluctuation amplitude with time through measurements of the gravitational infall of matter.
As will be presented in the analysis of this paper, the full suite of these complementary measurements allows a comprehensive assessment of the cosmological model.

In this section, we provide an overview of the cosmological model and a pedagogical summary of the observational signatures in BAO, RSD, CMB, SNe Ia, and weak lensing surveys that we use to provide new constraints on that model. This section is intended to provide the key details of the cosmological models and datasets that are explored in the remainder of the paper. The discussion will be familiar to the reader experienced in multiprobe cosmology constraints and will offer the highlights for additional study for the less experienced reader.

\section{A. Background models and notation}

Throughout this paper, we employ the standard cosmological model based on the Friedmann-Robertson-Walker metric where the scale factor, $a$, is unity today and is related to redshift by $a(t)=(1+z)^{-1}$. The evolution of the scale factor with time describes the background expansion history of the Universe, governed by the Friedmann equation, normally written as

$$
H^{2}(a)=\frac{8 \pi G}{3} \rho(a)-\frac{k c^{2}}{a^{2}} .
$$

$H \equiv \dot{a} / a$ is the Hubble parameter, and $\rho(a)$ is the total energy density (radiation + matter + dark energy). The curvature constant $k$ parametrizes the global curvature of space. An open universe is represented by $k<0$, and a closed universe is represented by $k>0$. The curvature term can be expressed in terms of an effective energy density through $-k c^{2} / a^{2}=(8 \pi G / 3) \rho_{k}(a)$. However, we note that a universe that is globally flat $(k=0)$ will appear to have a nonzero mean curvature due to horizon-scale fluctuations in the matter density field. These large-scale fluctuations place a fundamental limit on constraints on the curvature term under inflationary models that best describe CMB fluctuations, and the detectable limit is roughly one part in $10,000[86]$.

We define the dimensionless density parameter of each energy component $(x)$ by the ratio

$$
\Omega_{x}=\frac{\rho_{x}}{\rho_{\text {crit }}}=\frac{8 \pi G}{3 H^{2}} \rho_{x}
$$

so that $\sum \Omega_{x}=1$, where the sum is over all energy components including the curvature. Density parameters and $\rho_{\text {crit }}$ always refer to values at $z=0$ unless a dependence on $a$ or $z$ is stated explicitly, e.g., $\Omega_{x}(z)$. We will frequently refer to the present-day $\left(t_{0}\right)$ Hubble parameter $H_{0}$ through the dimensionless ratio $h \equiv H_{0} / 100 \mathrm{~km} \mathrm{~s}^{-1} \mathrm{Mpc}^{-1}$. The dimensionless quantity $\omega_{x} \equiv \Omega_{x} h^{2}$ is proportional to the physical density of component $x$ at the present day. 
The energy components considered in our models are pressureless (cold) dark matter (CDM), baryons, photons, neutrinos, and dark energy. The densities of CDM and baryons scale as $a^{-3}$; we refer to the density parameter of these two components together as $\Omega_{c b}$. The energy density in radiation $\left(\Omega_{r}\right)$ scales as $a^{-4}$; in the standard cosmological model, $\Omega_{r}$ is dynamically negligible in the low-redshift universe probed by spectroscopic surveys. However, the radiation density is dominant at very high redshift, where it receives contributions from the electromagnetic CMB radiation $\left(\omega_{\gamma}\right.$, known exquisitely well) and from neutrinos (at temperature $T$ higher than the rest energy $m_{\nu}$ ),

$\omega_{r}\left(T>m_{\nu}\right)=\omega_{\gamma}+\omega_{\nu}=\left[1+\frac{7}{8}\left(\frac{4}{11}\right)^{4 / 3} N_{\text {eff }}\right] \omega_{\gamma}$,

with $N_{\text {eff }}=3.044$ in the standard case with three neutrino species [87] (note: following precedent, we use $N_{\text {eff }}=$ 3.046 throughout, as done by Mangano et al. [88]). Other potential contributions to radiation energy density are traditionally parametrized in terms of their change to the effective number of neutrino species, $\Delta N_{\text {eff }}$, regardless of whether they represent extra neutrino species or other light degrees of freedom.

While the effect of neutrinos in cosmology has been detected through their contribution to the radiation energy density in the CMB [46], we have not yet reached the sensitivity to detect their mass. However, the detection of neutrino oscillations in terrestrial experiments strongly implies that at least two species are massive and that at least one species is now nonrelativistic (see Ref. [89] for a recent review). The energy density of neutrinos with nonzero mass scales like radiation at early times when the particles are ultrarelativistic. Once cosmic expansion reduces their kinetic energy below the rest mass, the neutrinos transition toward dark matter behavior. For neutrinos with nondegenerate mass eigenstates, the transition to nonrelativistic energies will happen at different epochs for the three eigenstates. In practice, for realistic neutrino masses, the transition occurs after the epoch of the last scattering in the $\mathrm{CMB}$, but before the epochs observed by spectroscopic surveys. Therefore, we can safely assume that the most massive neutrino species are ultrarelativistic at epochs relevant for the CMB and act as dark matter at epochs probed by galaxy surveys [90].

At the current level of precision, cosmological measurements are sensitive only to the sum of neutrino mass eigenvalues [90-94], thus allowing a simple modeling of neutrino masses with a single parameter, $\sum m_{\nu}$. We use $\nu \Lambda \mathrm{CDM}$ to refer to the flat $\Lambda \mathrm{CDM}$ model with this extra free parameter. Following standard convention, our total matter density at redshift $z=0$ therefore includes neutrinos, $\Omega_{m}=\Omega_{c b}+\Omega_{\nu}$.

Finally, the dark energy component is approximately constant in time and thus dominates the late-time evolution of the Universe (all the other components scale at least with $a^{-2}$ ). Conventionally, the dark energy component is parametrized in terms of its pressure-to-density ratio, $w=$ $p_{\mathrm{DE}} / \rho_{\mathrm{DE}}(c=1$ units $)$. We consider three basic phenomenological possibilities for $w$,

$$
w(a)=\left\{\begin{array}{l}
-1 \\
w \\
w_{0}+w_{a}(1-a) .
\end{array}\right.
$$

corresponding to cosmological constant, constant equation of state, and equation of state in the form of the CPL parametrization, respectively.

For the three cases in Eq. (4), the energy density of dark energy can be analytically integrated into

$$
\frac{\rho_{\mathrm{DE}}(a)}{\rho_{\mathrm{DE}, 0}}=\left\{\begin{array}{l}
1 \\
a^{-3(1+w)} \\
a^{-3\left(1+w_{0}+w_{a}\right)} \exp \left[-3 w_{a}(1-a)\right] .
\end{array}\right.
$$

We describe these models as $\Lambda \mathrm{CDM}, w \mathrm{CDM}$, and $w_{0} w_{a} \mathrm{CDM}$, respectively. By default, these models assume a flat geometry, but we also consider versions of these with free curvature. Dark energy models where $\Omega_{k}$ is allowed as a free parameter are referred to as $\mathrm{o} \Lambda \mathrm{CDM}$, owCDM, and $\mathrm{o} w_{0} w_{a} \mathrm{CDM}$. All of these models are nested in the sense that they contain $\Lambda \mathrm{CDM}$ as a special limit, with $w=w_{0}=-1, w_{a}=0$, and $\Omega_{k}=0$.

\section{B. Growth of structure in the cosmological model}

The cosmic expansion history is determined by the mean energy densities of the components in the Universe and their evolution with time. The structure growth history reflects the evolution of density perturbations against the background of cosmic expansion. Density perturbations in the matter are described by their relative perturbations

$$
\delta(\mathbf{x}, t) \equiv \frac{\rho_{m}(\mathbf{x}, t)-\bar{\rho}_{m}(t)}{\bar{\rho}_{m}(t)},
$$

where $\bar{\rho}_{m}(t)$ is the mean matter density of the Universe and $\mathbf{x}$ is the comoving coordinate. In this paper, we ignore theoretical subtleties related to choice of gauge because on the subhorizon scales of interest the Newtonian description is fully adequate.

To the first order in perturbation theory, the growth of fluctuations with time is specified by a single, scaleindependent growth factor, $D(t)$,

$$
\delta(\mathbf{x}, t)=D(t) \delta\left(\mathbf{x}, t_{0}\right),
$$

where $D\left(t_{0}\right)=1$ and $D(t)$ satisfies 


$$
\ddot{D}+2 H(z) \dot{D}-\frac{3}{2} \Omega_{m} H_{0}^{2}(1+z)^{3} D=0 .
$$

Strictly speaking, this equation only holds for a single fluid. However, it describes the low-redshift universe very well, since gravitational evolution drives the multiple fluids toward a common overdensity field. Therefore, in cosmological models consistent with GR, the growth of density fluctuations can be predicted uniquely for a given expansion history. In this work, we use growth measurements to probe dark energy, to measure the amplitude of the current matter density perturbations to test for tension in the cosmological model, and to test GR as the model for gravity on cosmological scales.

The linear growth rate is often expressed as a differential in the linear growth function with respect to the scale factor

$$
f(z) \equiv \frac{d \ln D}{d \ln a} .
$$

In standard cosmological models under GR, the growth rate can be approximated as $f(z) \propto \Omega_{m}(z)^{0.55}$ [95-97]. However, with the same expansion history, theories of modified gravity may predict different rates of structure growth, which motivates a simple parametrization to modifications to $f(z) \propto \Omega_{m}(z)^{\gamma}$, where departures from $\gamma=0.55$ correspond to departures from GR. Another strong prediction from GR is that the two metric potentials $\Psi$ and $\Phi$ (corresponding to time and space perturbations of the metric) are the same $(\Psi=\Phi)$. This is not necessarily so in theories of modified gravity, and the difference in the two potentials (known as gravitational slip) can affect the difference between the trajectories of relativistic and nonrelativistic particles.

In this work, we follow the analysis of Abbott et al. [98] to test for more general deviations from GR. Starting from scalar metric perturbations in the conformal Newtonian gauge, represented as $d s^{2}=a^{2}(\tau)\left[(1+2 \Psi) d \tau^{2}-\right.$ $\left.(1-2 \Phi) \delta_{i j} d x_{i} d x_{j}\right]$ with conformal time $\tau$ defined through $d \tau=d t / a(t)$, this phenomenological model allows modification to the Poisson equations or, equivalently, to the time variation of the gravitational constant and gravitational slip (e.g., Ref. [99]). A time-dependent parameter $\mu(a)$ plays a similar role as the $\gamma$ parameter in modifying the growth rate. The model also allows a perturbation of the potential for massless particles relative to matter particles through the time-dependent parameter $\Sigma(a)$. These two parameters provide linear perturbations to the GR form of gravity according to the relations

$$
\begin{gathered}
k^{2} \Psi=-4 \pi G a^{2}(1+\mu(a)) \rho \delta, \\
k^{2}(\Psi+\Phi)=-8 \pi G a^{2}(1+\Sigma(a)) \rho \delta,
\end{gathered}
$$

where $k$ is the wave number and $\delta$ is the comoving-gauge density perturbation. Both $\mu(a)$ and $\Sigma(a)$ are equal to zero at all redshifts in GR. This parametrization has the advantage that the $\Sigma$ term can be constrained independently by weak lensing with only mild degeneracy with $\mu$. The RSD measurements probe the response of matter to a gravitational potential and therefore provide independent constraints on the $\mu$ term. Again, following Abbott et al. [98], we describe the redshift evolution of $\mu$ and $\Sigma$ as

$$
\mu(z)=\mu_{0} \frac{\Omega_{\Lambda}(z)}{\Omega_{\Lambda}}, \quad \Sigma(z)=\Sigma_{0} \frac{\Omega_{\Lambda}(z)}{\Omega_{\Lambda}} .
$$

Finally, neutrinos can affect the measured growth of fluctuations. While ultrarelativistic, they freestream out of overdensities and thus suppress growth on scales smaller than their freestreaming length (e.g., Ref. [90]). The dominant effect is a decrease in the amplitude of fluctuations at low redshifts compared to extrapolations from the $\mathrm{CMB}$ under a model with zero neutrino mass.

\section{Observable signatures}

\section{1. $C M B$}

The temperature of the CMB is uniform across the sky to one part in 100,000; beyond this level, anisotropies appear at all observable scales. The angular power spectrum of the $\mathrm{CMB}$ can be predicted to high precision based on an inflationary model and an expansion model. The fluctuation modes corresponding to scales greater than one degree were larger than the Hubble distance at the time of the last scattering and capture the initial conditions imprinted at the end of inflation ( $n_{s}$ and its derivative). At smaller scales, the sound waves that propagate in the ionized universe due to photon-baryon coupling imprint the characteristic acoustic oscillations into the $\mathrm{CMB}$ power spectrum. The relative amplitudes of the peaks of the oscillations provide information on the energy contents of the Universe, while the spacing of the peaks provides a BAO "standard ruler" whose length can be computed using straightforward physics.

Using the latest CMB results under an assumption of a $\Lambda \mathrm{CDM}$ cosmology, the BAO feature has a comoving scale of $147.18 \pm 0.29 \mathrm{Mpc}$ [46], set by the distance $r_{d}$ traveled by sound waves between the end of inflation and the decoupling of baryons from photons after recombination,

$$
r_{d}=\int_{z_{d}}^{\infty} \frac{c_{s}(z)}{H(z)} d z
$$

where $z_{d}$ is the redshift of the drag epoch and $c_{s}$ is the sound speed. Not to be confused with the redshift at the time of last scattering, the drag epoch corresponds to the time when the baryons decouple from the photons, around a redshift $z=1020$. In the standard cosmological models explored here, $r_{d}$ can be computed given the physical densities of dark matter $\left(\omega_{c}\right)$, baryonic matter 
$\left(\omega_{b}\right)$, and the radiation content of the Universe. The radiation content can be determined from the temperature of the CMB and the effective number of neutrino species $\left(N_{\text {eff }}\right)$. Combined, these abundances determine the shape and position of the BAO peak in comoving space that can then be used as a standard ruler. Because the $\mathrm{CMB}$ provides an image of the oscillations at the epoch of last photon scattering, the BAO scale has not reached its maximum size, but it can still be measured at very high precision to provide a constraint on the angular diameter distance to a redshift of $z \sim 1100$.

Because the proton-electron plasma does not recombine instantaneously, the last scattering surface has a finite thickness. Photon diffusion also results in damping at the smallest scales, leading to a diffusion scale that depends on the expansion rate and energy densities. The effect of damping on the power spectrum therefore allows constraints on the energy densities of relativistic particles, primordial helium abundance, dark matter, and baryon matter at the time of last scattering. Finally, the signal from the $\mathrm{CMB}$ records the integrated ionization history of hydrogen and the integrated formation of structure in the form of polarization and lensing signals. Characterization of polarization and lensing in the CMB thus provides information about the integrated optical depth $(\tau)$ to the surface of last scattering and the effects of neutrinos on the growth rate of structure. One of the main challenges in interpreting the high signal-to-noise $\mathrm{CMB}$ data is the modeling of foreground contamination, whether from dust emission, induced polarization, or radio sources. For a review of experimental and analysis methods to extract cosmological information from the CMB, see the work by Staggs et al. [100] and the Planck Collaboration [101].

\section{BAO measurements from spectroscopic surveys}

The same sound waves that appear as acoustic oscillations in the CMB appear in the clustering of matter at later times, although with a weaker amplitude due to the coupling of baryonic matter with dark matter (e.g., Ref. [102]). For this reason, survey volumes of several $\mathrm{Gpc}^{3}$ are required to reach percent-level precision constraints on the BAO feature. The dark matter distribution that records the $\mathrm{BAO}$ feature cannot be probed directly and is instead traced by galaxies, quasars, or absorption line systems corresponding to neutral hydrogen or other material in the intergalactic medium.

The cosmological parameters used to calibrate the characteristic BAO scale $r_{d}$ are typically derived from CMB observations. The $r_{d}$ scale can also be derived from big bang nucleosynthesis (BBN) measurements (giving constraints on $\omega_{b}$ ) in combination with measurements of expansion history (giving constraints on $\Omega_{m}$ ), if the early Universe is assumed to be a mixture of radiation, baryonic matter, and cold dark matter with three neutrino species. With a calibrated $r_{d}$, the BAO scale can be used to make absolute distance measurements as a function of redshift. Or $r_{d}$ can be treated as a nuisance parameter, allowing multiple $\mathrm{BAO}$ measurements over a range of redshifts to be used for relative measures of the cosmic expansion history.

In a spectroscopic survey, the BAO feature appears in both the line-of-sight direction and the transverse direction. Along the line-of-sight direction, a measurement of the redshift interval, $\Delta z$, over which the BAO feature extends, provides a means to directly measure the Hubble parameter, $H(z)=c \Delta z / r_{d}$. Equivalently, it measures the Hubble distance at redshift $z$,

$$
D_{H}(z)=\frac{c}{H(z)} .
$$

Along the transverse direction, the BAO scale corresponds to an angle, $r_{d}=D_{M}(z) \Delta \theta$. Measuring the angle $\Delta \theta$ subtended by the BAO feature at a given redshift provides a means to estimate the (comoving) angular diameter distance, $D_{M}(z)$, which depends on the expansion history and curvature as

$$
D_{M}(z)=\frac{c}{H_{0}} S_{k}\left(\frac{D_{C}(z)}{c / H_{0}}\right) .
$$

Here, the line-of-sight comoving distance is

$$
D_{C}(z)=\frac{c}{H_{0}} \int_{0}^{z} d z^{\prime} \frac{H_{0}}{H\left(z^{\prime}\right)}
$$

and

$$
S_{k}(x)= \begin{cases}\sin \left(\sqrt{-\Omega_{k}} x\right) / \sqrt{-\Omega_{k}} & \Omega_{k}<0, \\ x & \Omega_{k}=0, \\ \sinh \left(\sqrt{\Omega_{k}} x\right) / \sqrt{\Omega_{k}} & \Omega_{k}>0 .\end{cases}
$$

When considering the dependence of $r_{d}$ on cosmology, the quantities that the BAO measurements directly constrain are $D_{M}(z) / r_{d}$ and $D_{H}(z) / r_{d}$. The BAO measurements were also historically summarized by a single quantity representing the spherically averaged distance,

$$
D_{V}(z) \equiv\left[z D_{M}^{2}(z) D_{H}(z)\right]^{1 / 3},
$$

or more directly $D_{V}(z) / r_{d}$. The powers of $2 / 3$ and $1 / 3$ approximately account for two transverse and one radial dimensions, and the extra factor of $z$ is a conventional normalization. Today, we almost always specify the transverse and radial $\mathrm{BAO}$ as two independent measurements with correlated error bars instead, unless the signal-to-noise ratio is low.

For measurements using discrete tracers with sufficiently high number density, the BAO feature in clustering measurements can be sharpened through a process known as "reconstruction" [103]. Reconstruction uses the observed three-dimensional map of galaxy positions to infer 
their peculiar velocities. Each galaxy tracer is then moved to a position that is approximately where the galaxy would reside if there were no bulk flows. The process removes the dominant nonlinear effect from the BAO feature, which is smearing caused by the large-scale bulk flows. Reconstruction recovers almost all theoretically available information in the BAO. In the SDSS analyses, the fitting to reconstructed data is performed with minimal information from the broadband clustering signal, in an attempt to isolate the $\mathrm{BAO}$ signal.

A review of BAO as a probe for cosmology is presented in Sec. 4 of Ref. [104], and a discussion on the BAO measurement in practice can be found in the Appendix A.

\section{RSD measurements from galaxy surveys}

The galaxy redshifts used in spectroscopic BAO measurements can also be used to study anisotropic clustering. There are two primary ways in which anisotropy is introduced into the large-scale clustering of matter: the Alcock-Paczynski (AP) effect [105] and the RSD effect from the growth of structure [106]. The AP effect arises in clustering statistics as a deviation from physically isotropic signal due to an incorrect translation of angular and radial (redshift) separations to physical ones (e.g., from lack of knowledge of the true cosmology; see Appendix A). The AP effect thus serves as a way to measure the product of $H(z)$ and $D_{M}(z)$, offering additional constraints on dark energy and curvature (e.g., Ref. [107]).

The RSD effect arises from the growth of structure [106] and is observed due to the bulk flow of matter in response to the gravitational potential of matter overdensities. The peculiar velocities introduce additional redshifts on top of those caused by cosmic expansion, leading to an increase in the measured amplitude of radial clustering relative to transverse clustering on large scales. The resulting anisotropy is correlated with the rate at which structure grows. The growth rate $f(z)$ from Eq. (9) can also be expressed as

$$
f=\frac{\partial \ln \sigma_{8}}{\partial \ln a},
$$

where $\sigma_{8}(z)$ describes the amplitude of linear matter fluctuations on a comoving scale of $8 h^{-1} \mathrm{Mpc}$. The RSD measurements provide constraints on $f \sigma_{8}$, which characterizes the amplitude of the velocity power spectrum.

The AP and RSD signals are partially degenerate, which limits the AP signal that can be extracted from measurements of clustering (e.g., Ref. [108]). A review of RSD and $\mathrm{AP}$ as a probe for cosmology is presented in Sec. 4 of Ref. [104], while a discussion of the RSD measurement in practice can be found in Appendix A.

\section{Weak lensing}

As RSD probe the response of matter to a gravitational potential, gravitational lensing probes the response of photons to a gravitational potential. Gravitational lensing can be observed in several forms in cosmic surveys, and we focus on the weak lensing regime in this work. More specifically, we use cosmic shear measurements of weak lensing and galaxy-galaxy lensing measurements in performing cosmological constraints.

Cosmic shear shows up as distortions on the order of $1 \%$ that appear in the images of background galaxies due to lensing by the integrated foreground mass distribution. By introducing correlations of neighboring galaxy shapes due to shared foregrounds, cosmic shear allows direct inference of the gravitational potential gradients integrated along the line of sight. If these correlations are computed over discrete intervals over a range of redshifts, a smooth, three-dimensional mapping of the matter distribution can be deduced. The direct observable in lensing surveys is the cosmic shear power spectrum, with an amplitude that scales approximately as $\Omega_{m}^{2} \sigma_{8}^{2}$ in the linear regime. However, weak-lensing measurements are often in the nonlinear regime and also depend on relative distances through the lens equation. The relative balance between $\Omega_{m}$ and $\sigma_{8}$ in the measurement depends on a number of factors within CDM models, as described by Jain and Seljak [109]. For the redshifts probed by current surveys, around the benchmark $\Lambda$ CDM model, the redshift evolution of the amplitude of the cosmic shear power spectrum is best described by the approximate combination

$$
S_{8} \equiv \sigma_{8}\left(\Omega_{m} / 0.3\right)^{0.5}
$$

A review of cosmic shear methodology and its challenges as a probe for cosmology can be found in Sec. 5 of Ref. [104].

In addition to shear measurements, we also use galaxygalaxy lensing results in Sec. VI to provide additional information on the galaxy clustering measurements obtained in photometric surveys. Galaxy-galaxy lensing measurements probe the local gravitational potential around specific classes of galaxies. For the cosmology studies presented here, these measurements give insight into mass density profiles, thus providing important information on the bias of the galaxies used as tracers in the photometric clustering measurements.

\section{Type Ia supernovae}

Type Ia supernovae are generally believed to occur when a white dwarf approaches the Chandrasekhar mass limit due to mass accretion or merger. This class of $\mathrm{SN}$ is easily characterized with spectroscopy due to the strong calcium and silicon lines and lack of hydrogen and helium lines. While SNe Ia are not perfect standard candles, their diversity can be described by the SN light curve width (hereafter $X_{1}$ ) and $\mathrm{SN}$ color at maximum brightness (hereafter $C$ ). The distance modulus, $\mu=5 \log _{10}\left[D_{L}(z) / 10 \mathrm{pc}\right]$, is then given by 
TABLE I. Symbols and definitions of cosmological parameters.

\begin{tabular}{ll}
\hline \hline Parameter & \multicolumn{1}{c}{ Definition } \\
\hline$\Omega_{m}$ & Density parameter of matter \\
$\Omega_{c}$ & Density parameter of cold dark matter \\
$\Omega_{b}$ & Density parameter of baryons \\
$\Omega_{\Lambda}$ & Density parameter of cosmological constant \\
$\Omega_{\mathrm{DE}}$ & Density parameter of dark energy \\
$\Omega_{k}$ & Curvature parameter \\
$\omega_{c}=\Omega_{c} h^{2}$ & Physical density parameter of cold dark matter \\
$\omega_{b}=\Omega_{b} h^{2}$ & Physical density parameter of baryons \\
$H_{0}$ & Current expansion rate (Hubble constant) \\
$h$ & Ho $/ 100$ km s ${ }^{-1}$ Mpc \\
$\theta_{\mathrm{MC}}$ & Approximate angular scale of sound horizon (COSMOMC) \\
$A_{s}$ & Power of the primordial curvature perturbations at $k=0.05 \mathrm{Mpc}{ }^{-1}$ \\
$\sigma_{8}$ & Amplitude of matter fluctuation on $8 h^{-1} \mathrm{Mpc}$ comoving scale \\
$n_{s}$ & Power-law index of the scalar spectrum \\
$\tau$ & Thomson scattering optical depth due to reionization \\
$N_{\mathrm{eff}}$ & Effective number of neutrinolike relativistic degrees of freedom \\
$w\left(w_{0}\right)$ & Dark energy equation of state, $w=p_{\mathrm{DE}} / \rho_{\mathrm{DE}}(c=1$ units) \\
$w_{a}$ & Time derivative of dark energy equation of state parameter [Eq. (4)] \\
$\sum m_{\nu}$ & Sum of neutrino masses \\
\hline \hline
\end{tabular}

$$
\mu=m_{B}^{*}-\left(M_{B}-\alpha X_{1}+\beta C\right),
$$

where $m_{B}^{*}$ is the observed SN peak magnitude in the restframe $B$ band [110]. Here, $D_{L}$ is the luminosity distance, which follows the relation $D_{L}=D_{M}(1+z)$. The quantity $M_{B}$ characterizes the SN Ia absolute magnitude, while $\alpha$ and $\beta$ describe the change in magnitude with diversity in width and color, respectively. The linear dependence between SN property and peak magnitude follows from the empirical observation that brighter $\mathrm{SNe}$ Ia are also slower to rise and/or bluer in color (see Refs. [111,112]). Beyond those two dominant effects, a residual diversity related to host galaxy properties was also found (e.g., Ref. [25]), with brighter $\mathrm{SNe}$ occurring in more massive galaxies. This effect is usually accounted for by considering that the SN Ia absolute magnitude is different depending on the host stellar mass, such as in Ref. [113]:

$$
\begin{cases}M_{B}=M_{B}^{1} & \text { if } M_{\text {stellar }}<10^{10} M_{\odot} \\ M_{B}=M_{B}^{1}+\Delta_{M} & \text { otherwise. }\end{cases}
$$

The model assumes that SNe Ia with identical color, light curve shape, and galactic environment have on average the same intrinsic luminosity for all redshifts. Note that the hypothesis of redshift independence can be checked with data for $\Delta_{M}, \alpha$, and $\beta$ and so far has been found to be consistent with observations (e.g., Ref. [114]).

If the above model is sufficiently accurate, the measured SN distance modulus traces the redshift dependence of luminosity distance. The absolute magnitude can be calibrated using nearby SNe Ia and Cepheid variables, giving a distance ladder from which $H_{0}$ can be computed. A review of supernova astrophysics and their use in cosmology to constrain the dark energy equation of state can be found in Ref. [115].

\section{Combining measurements}

The measurements of the redshift-distance relation through $\mathrm{BAO}, \mathrm{AP}$, and $\mathrm{SNe}$ Ia provide tests of extended models for dark energy and cosmic expansion that are only weakly constrained with $\mathrm{CMB}$ data alone. Generally speaking, the $\mathrm{SNe}$ Ia data provide a high-precision constraint of the luminosity distance-redshift relation in the dark-energy dominated regime while the $\mathrm{BAO}$ and $\mathrm{AP}$ measurements sample the matter-dominated regime and the epoch of matterdark energy equality. Likewise, the measurements of growth of structure through RSD and weak lensing allow additional tests on the background expansion and on whether GR describes the rate of structure growth. Measurements of the redshift-distance relation and growth of structure allow tests of the neutrino mass by constraining the effects on both the cosmic expansion after the CMB formation and the amplitude of matter fluctuations relative to amplitude of CMB fluctuations. The sensitivity of the latter approach is limited by our knowledge of optical depth $\tau$ to the last scattering surface. Alternative approaches to constrain the neutrino mass rely on measuring the redshift-dependence of growth directly with clustering data or scale dependence of the matter power spectrum $[90,116,117]$ but are not explored here.

For fitting the measurements, model calculations throughout this paper are made with COSMOMC [118]. Figures are produced with the GetDist PYTHON package [119]. The model parameters are summarized in Table I, while parametrizations and priors are described in Appendix B. We stress that choice of parametrization is 
TABLE II. Datasets for cosmology analyses.

\begin{tabular}{llr}
\hline \hline Name & Data combination & Cosmology analysis \\
\hline BAO & $D_{M}(z) / r_{d}$ and $D_{H}(z) / r_{d}$ from BAO measurements of all SDSS tracers & Sec. IV \\
RSD & $f \sigma_{8}(z)$ from all SDSS tracers, marginalizing over $D_{M}(z) / r_{d}$ and $D_{H}(z) / r_{d}$ & Sec. V \\
SDSS & $D_{M}(z) / r_{d}, D_{H}(z) / r_{d}$, and $f \sigma_{8}(z)$ of all SDSS tracers & Secs. VI, VII \\
CMB T\&P & Planck TT, TE, EE, and lowE power spectra & Secs. IV, V \\
CMB lens & Planck lensing measurements & Sec. V \\
Planck & Planck temperature, polarization, and lensing measurements & Secs. VI, VII \\
SN & Pantheon SNe Ia measurements & Secs. IV, VI, VII \\
WL & DES cosmic shear correlation functions & Sec. V \\
DES & DES $3 \times 2$ measurements (cosmic shear, galaxy clustering, and galaxy-galaxy lensing) & Secs. VI, VII \\
\hline \hline
\end{tabular}

sometimes important-the shape and visual overlap of marginalized contours can be significantly impacted, especially in a prior-dominated regime. In all cases that use information from the shape of the power spectrum, we hold $N_{\text {eff }}$ fixed to its baseline value. In the majority of the studies presented in this paper, the priors we assume on free parameters do not impact the posterior distributions when CMB data are included in the likelihoods. We refer to this series of priors as those with the "CMB" parametrization. In the cases where we study the expansion history without the CMB (Sec. IV), we use the "background" parametrization. In all studies, the same priors are used for curvature, the dark energy equation of state, or neutrino masses in the cases in which those parameters are fit to the data. Those priors are reported in the "extended" portion of the table in Appendix B.

\section{DATA AND METHODOLOGY}

In this section, we provide an overview of the different measurements used in our primary cosmological analysis, including $\mathrm{BAO}, \mathrm{RSD}, \mathrm{CMB}, \mathrm{SNe}$, and weak lensing (WL). The samples we use in this work and the naming conventions we choose are summarized in Table II. We present the state-of-the-art results and discuss how the different probes have evolved during the last decade.

\section{A. SDSS BAO and RSD measurements}

The study presented in this work characterizes the impact of BAO and RSD measurements from spectroscopic galaxy and quasar samples obtained over four generations of SDSS. A summary of the BAO-only measurements is found in Table III and in the top panel of Fig. 1. In these

TABLE III. Clustering measurements ${ }^{\mathrm{a}}$ for each of the BAO and RSD samples used in this paper.

\begin{tabular}{|c|c|c|c|c|c|c|c|c|}
\hline Parameter & MGS & BOSS galaxy & BOSS galaxy & eBOSS LRG & eBOSS ELG & eBOSS quasar & $\operatorname{Ly} \alpha-\operatorname{Ly} \alpha$ & Ly $\alpha$-quasar \\
\hline \multicolumn{9}{|c|}{ Sample properties } \\
\hline Redshift range & $0.07<z<0.2$ & $0.2<z<0.5$ & $0.4<z<0.6$ & $0.6<z<1.0$ & $0.6<z<1.1$ & $0.8<z<2.2$ & $z>2.1$ & $z>1.77$ \\
\hline$N_{\text {tracers }}$ & 63,163 & 604,001 & 686,370 & 377,458 & 173,736 & 343,708 & $210,005^{\mathrm{b}}$ & $341,468^{\mathrm{c}}$ \\
\hline$z_{\text {eff }}$ & 0.15 & 0.38 & 0.51 & 0.70 & 0.85 & 1.48 & 2.33 & 2.33 \\
\hline$V_{\text {eff }}\left(\mathrm{Gpc}^{3}\right)^{\mathrm{d}}$ & 0.24 & 3.7 & 4.2 & 2.7 & 0.6 & 0.6 & & \\
\hline \multicolumn{9}{|c|}{ BAO-only measurements ${ }^{\mathrm{e}}$ (Sec. IV) } \\
\hline$D_{V}(z) / r_{\mathrm{d}}$ & $4.47 \pm 0.17$ & & & & $18.33_{-0.62}^{+0.57}$ & & & \\
\hline$D_{M}(z) / r_{\mathrm{d}}$ & & $10.23 \pm 0.17$ & $13.36 \pm 0.21$ & $17.86 \pm 0.33$ & & $30.69 \pm 0.80$ & $37.6 \pm 1.9$ & $37.3 \pm 1.7$ \\
\hline$D_{H}(z) / r_{\mathrm{d}}$ & & $25.00 \pm 0.76$ & $22.33 \pm 0.58$ & $19.33 \pm 0.53$ & & $13.26 \pm 0.55$ & $8.93 \pm 0.28$ & $9.08 \pm 0.34$ \\
\hline \multicolumn{9}{|c|}{ RSD-only measurements (Sec. V) } \\
\hline$f \sigma_{8}(z)$ & $0.53 \pm 0.16$ & $0.500 \pm 0.047$ & $0.455 \pm 0.039$ & $0.448 \pm 0.043$ & $0.315 \pm 0.095$ & $0.462 \pm 0.045$ & & \\
\hline \multicolumn{9}{|c|}{ BAO + RSD measurements (Secs. VI and VII) } \\
\hline$D_{V}(z) / r_{\mathrm{d}}$ & $4.51 \pm 0.14$ & & & & & & & \\
\hline$D_{M}(z) / r_{\mathrm{d}}$ & & $10.27 \pm 0.15$ & $13.38 \pm 0.18$ & $17.65 \pm 0.30$ & $19.5 \pm 1.0$ & $30.21 \pm 0.79$ & $37.6 \pm 1.9$ & $37.3 \pm 1.7$ \\
\hline$D_{H}(z) / r_{\mathrm{d}}$ & & $24.89 \pm 0.58$ & $22.43 \pm 0.48$ & $19.78 \pm 0.46$ & $19.6 \pm 2.1$ & $13.23 \pm 0.47$ & $8.93 \pm 0.28$ & $9.08 \pm 0.34$ \\
\hline$f \sigma_{8}(z)$ & $0.53 \pm 0.16$ & $0.497 \pm 0.045$ & $0.459 \pm 0.038$ & $0.473 \pm 0.041$ & $0.315 \pm 0.095$ & $0.462 \pm 0.045$ & & \\
\hline
\end{tabular}

${ }^{\mathrm{a}}$ Uncertainties are Gaussian approximations to the likelihoods for each tracer ignoring the correlations between measurements.

${ }^{\mathrm{b}}$ The number of tracers reported for the $\operatorname{Ly} \alpha$-Ly $\alpha$ measurement corresponds to the number of sightlines, or forests.

${ }^{\mathrm{c}}$ Number of tracer quasars is used for the Ly $\alpha$-quasar study.

${ }^{\mathrm{d}}$ The effective volume, $V_{\text {eff }}$, is quoted here in $\mathrm{Gpc}^{3}$ using a flat $\Lambda \mathrm{CDM}$ model with $\Omega_{m}=0.31$ and $h=0.676$.

${ }^{\mathrm{e}}$ The measurements for MGS, the two BOSS galaxy samples, eBOSS LRG, and eBOSS ELG are performed after reconstruction. 


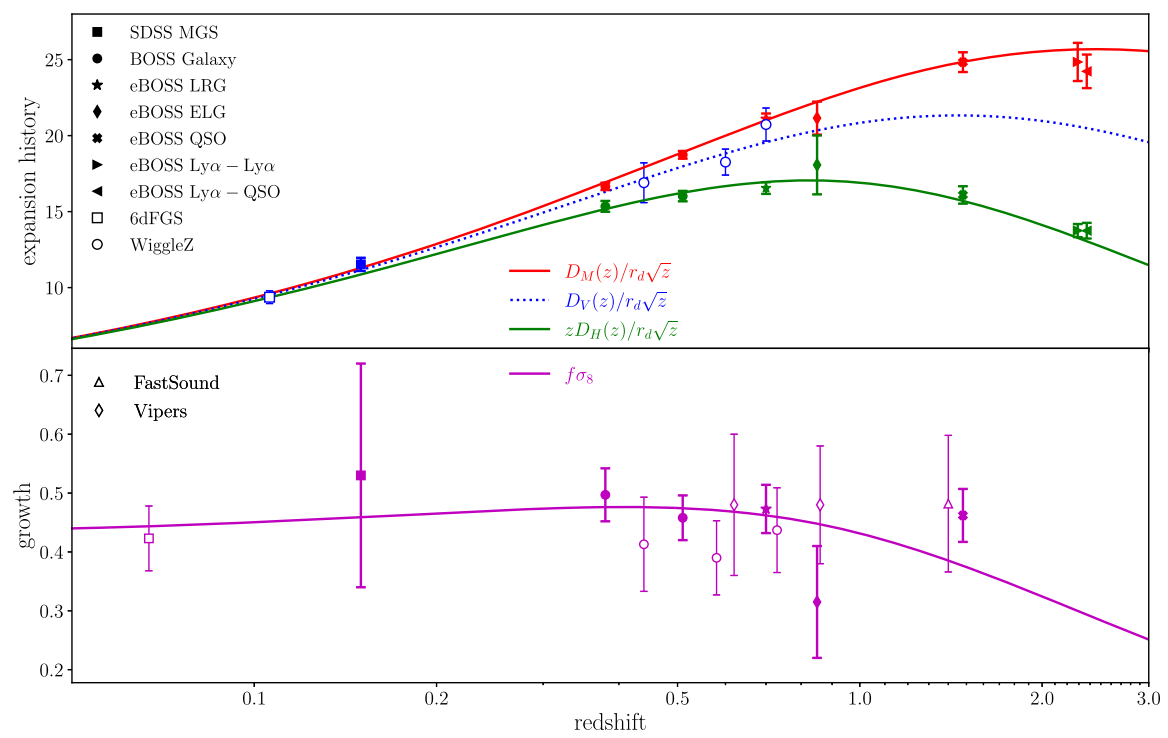

FIG. 1. Top: distance measurements from the SDSS lineage of BAO measurements presented as a function of redshift. Measurements include those from SDSS MGS [120,121], BOSS galaxies [57], eBOSS LRGs [68,69], eBOSS ELGs [71,72], eBOSS quasars [73,74], the BOSS + eBOSS Ly $\alpha$ autocorrelation, and the BOSS + eBOSS Ly $\alpha$-quasar cross-correlation measurements [75]. Also shown are the BAO measurements from 6dFGS [51] and WiggleZ [53] for comparison to measurements from other redshift surveys. Red points correspond to transverse $\mathrm{BAO}$, green points correspond to radial $\mathrm{BAO}$, and blue corresponds to an isotropic $\mathrm{BAO}$ measurement. The red, green, and blue theory curves are not fit to the BAO data; they are the Planck best-fit predictions for a flat $\Lambda$ CDM model. Bottom: growth rate measurements from the SDSS lineage of $f \sigma_{8}$ measurements as a function of redshift. The measurements match the BAO samples except for $z>2$, where we do not report a measurement of the growth rate. In addition to the SDSS measurements, we include the FastSound [122], Vipers [123], 6dFGS [124], and WiggleZ measurements for comparison. As for the upper panel, the theory curve is not a fit, but a best-fit Planck model.

measurements, the broadband clustering signal that carries information on the AP effect or RSD is effectively deweighted to capture only the BAO signature. These measurements are used to explore the impact of BAO measurements on models for dark energy in Sec. IV. Results from the full-shape fits, without information from reconstructed $\mathrm{BAO}$ measurements, are found in the central region of Table III. These measurements include information from the AP effect and are used to explore the impact of growth measurements in Sec. VA summary of the BAO and RSD measurements, including information from the AP effect and reconstruction, is also found in Table III and Fig. 1. These measurements are used to perform the global cosmology fitting in Secs. VI and VII. The background to each of these measurements is summarized below and described in detail in the relevant references. All results in Table III reflect the consensus values in the cases where multiple measurements are made.

In this paper, we only include large-scale structure based measurements from SDSS experiments. These are consistent with those from other experiments, including 6dFGS [51] and WiggleZ [53]. However, the non-SDSS experiments do not add significantly to the measurements from SDSS: for example, Carter et al. [125] showed that 6dFGS only adds enough information to provide an improvement of approximately $16 \%$ on the SDSS main galaxy sample (MGS) results at low redshift, while the WiggleZ sample has less than $10 \%$ of the effective volume of the BOSS CMASS sample (see Table 1 of Ref. [126]). Given issues with overlap between samples and the resulting complicated covariance, we simply do not include these data.

\section{Main galaxy sample $(0.07<z<0.2)$}

The first two generations of SDSS (SDSS-I and -II) provided redshifts of nearly one million galaxies [127]. SDSS galaxies were selected with $14.5<r<17.6$ [128] over a contiguous footprint of $6813 \mathrm{deg}^{2}$ to perform clustering measurements. The sample was further refined to cover the redshift range $0.07<z<0.2$, include the bright objects with $M_{r}<-21.2$, and include red objects with $g-r>0.8$. The resulting sample contains 63,163 galaxies intended to occupy the highest mass halos while providing a roughly uniform number density over the full redshift interval. The sample was used to perform a BAO measurement from the reconstructed correlation function [120] and an RSD measurement from the anisotropic correlation function [121], both at an effective redshift $z_{\text {eff }}=0.15$. The BAO measurement was characterized with $D_{V}(z) / r_{d}$ and the RSD fit was performed using the postreconstruction BAO fit as a prior. The likelihoods from this work are found in the Supplementary Data associated with Ref. [121]. We refer to this sample as the "main galaxy sample" in the table and throughout the paper. 


\section{BOSS DR12 galaxies $(0.2<z<0.6)$}

Over the period 2009-2014, BOSS performed spectroscopy to measure large-scale structure with galaxies over the redshift interval $0.2<z<0.75$. BOSS obtained redshifts for $1,372,737$ galaxies over $9,376 \mathrm{deg}^{2}$ from which the final galaxy catalog was produced for clustering measurements [129]. The sample was divided into three redshift bins covering $0.2<z<0.5,0.4<z<0.6$, and $0.5<z<$ 0.75 for studies of BAO and RSD. For each redshift bin, seven different measurements of BAO, AP, and RSD were performed [130-136] based on the galaxy correlation function or power spectrum. Following the methodology of Sánchez et al. [137], these measurements were combined into a single consensus likelihood spanning $D_{M}(z) / r_{d}$ and $D_{H}(z) / r_{d}$ for the BAO-only measurements and $D_{M}(z) / r_{d}$, $D_{H}(z) / r_{d}$, and $f \sigma_{8}(z)$ for the combined BAO and RSD measurements. These results were computed over all three redshift intervals after fully accounting for systematic errors and covariances between parameters and between redshift bins [57]. We refer to the $0.2<z<0.5$ and $0.4<$ $z<0.6$ samples as the "BOSS galaxies."

\section{3. eBOSS galaxies and quasars $(0.6<z<2.2)$}

EBOSS began full operations in July 2014 to perform spectroscopy on LRGs, ELGs, and quasars and concluded on March 1, 2019. EBOSS obtained reliable redshifts for 174,816 LRGs over the interval $0.6<z<1$ in an area of $4,103 \mathrm{deg}^{2}$. The targets for spectroscopy were selected from SDSS riz imaging data and infrared sky maps from the Wide-Field Infrared Survey Explorer (WISE) [138]. The LRG selection [139] was optimized to cover $0.6<$ $z<1$ with a median redshift $z=0.72$. The sample was supplemented with the galaxies in the $z>0.6$ tail of the BOSS DR12 redshift distribution, but over the full $9,376 \mathrm{deg}^{2}$ of the BOSS footprint. The addition of BOSS galaxies more than doubles the total sample size to 377,458 redshifts while slightly lowering the effective redshift. This "eBOSS LRG" sample was used to measure $D_{M}(z) / r_{d}$ and $D_{H}(z) / r_{d}$ using a catalog of reconstructed galaxy positions. In addition, the sample was used to perform a joint $D_{M}(z) / r_{d}, D_{H}(z) / r_{d}$, and $f \sigma_{8}(z)$ measurement in both the correlation function [68] and the power spectrum [69].

Covering an area of 1, $170 \mathrm{deg}^{2}$, eBOSS also obtained reliable redshifts for 173,736 ELGs over the redshift range $0.6<z<1.1$. These targets were identified in grz photometry from the Dark Energy Camera (DECam) [140] following the selection algorithms presented by Raichoor et al. [141]. These star-forming galaxies were spectroscopically confirmed with high efficiency due to their strong emission lines that are easily detectable with the BOSS spectrographs [65] to $z=1.1$. The "eBOSS ELG" sample reaches an effective redshift $z_{\text {eff }}=0.85$. We performed an isotropic BAO fit to measure $D_{V}(z) / r_{d}$
[70,72] and a combined RSD and BAO analysis to constrain $f \sigma_{8}(z), D_{H}(z) / r_{d}$ and $D_{M}(z) / r_{d}$ from both the correlation function [71] and the power spectrum [72]. Because the likelihoods are not well described by a Gaussian distribution, we use the full likelihoods in the cosmology fitting.

Finally, the "eBOSS quasar" sample includes 343,708 reliable redshifts with $0.8<z<2.2$ over $4,699 \mathrm{deg}^{2}$. The sample selection [142] was derived from WISE infrared and SDSS optical imaging data; $18 \%$ of these quasars identified by the algorithm had been observed in SDSS-I, -II, or -III. The sample was used to measure $D_{M}(z) / r_{d}, D_{H}(z) / r_{d}$, and $f \sigma_{8}(z)$ from both the correlation function [73] and the power spectrum [74]. The consensus BAO-only results were determined without reconstruction. The full-shape $D_{M}(z) / r_{d}, D_{H}(z) / r_{d}$, and $f \sigma_{8}(z)$ measurements were therefore not combined with the BAO-only measurements.

\section{Lyman- $\alpha$ forest samples $(1.8<z<3.5)$}

The complete BOSS sample contains the spectra of 157,845 quasars at $2.0<z<3.5$ that are free of significant broad absorption lines. These quasar targets were selected using a variety of techniques [143] to measure fluctuations in the transmission of the Lyman- $\alpha(\operatorname{Ly} \alpha)$ forest due to fluctuations in the density of neutral hydrogen. The autocorrelation of the Ly $\alpha$ forest and its cross-correlation with 217,780 quasars at $1.8<z<3.5$ led to $2 \%$ precision measurements of the $\mathrm{BAO}$ distance scale at $z_{\mathrm{eff}}=2.33[58,59]$.

Several techniques, such as those using photometric variability [144], were used to select new $z>2.1$ quasars to observe in eBOSS. In addition, 42,859 quasars with low signal-to-noise BOSS spectra were reobserved in eBOSS to better measure the fluctuations in the Ly $\alpha$ forest. Finally, improvements to the analysis methods enabled the use of a larger wavelength range for determining the forest. The final sample used to trace the $\operatorname{Ly} \alpha$ forest has 210,005 quasars at $z>2.1$, consisting of the original sample from BOSS and the sample from eBOSS. A total of 341,468 quasars with $z>1.77$ were used for cross-correlation studies with the Ly $\alpha$ forest.

The final eBOSS results are presented by du Mas des Bourboux et al. [75]. The auto- and cross-correlation measurements can also be combined into a single estimate of $D_{M}(z) / r_{d}$ and $D_{H}(z) / r_{d}$ with associated covariances [75], resulting in a $25 \%$ reduction in the area of the contours relative to the BOSS DR12 studies. The uncertainties quoted in Table III correspond to a Gaussian approximation of the real likelihood, but in our analysis, we use the full (non-Gaussian) likelihood.

In Ref. [75], we also presented a 4\% measurement of the redshift-space distortion parameter of the Ly $\alpha$ forest, $\beta_{F}$. However, $\beta_{F}$ cannot be readily translated into a measurement of $f \sigma_{8}$, since the response of Ly $\alpha$ forest fluctuations to a velocity gradient is unknown $[145,146]$. 


\section{Summary of SDSS measurements and systematic errors}

From the BOSS and eBOSS clustering analyses with galaxies and quasars, the main systematic errors in BAO and RSD estimates arise from modeling of the two-point statistics, the choice of fiducial cosmology taken as a reference for coordinate transformation and power spectrum template, and from the observational effects. The systematic errors also have larger effect on the RSD analyses than the BAO analyses. The estimation of the systematic errors was done in a similar fashion for all tracers, although some differences in the treatment remain and are outlined in the following.

The modeling systematic errors are studied using accurate mocks based on $\mathrm{N}$-body simulations for which the cosmology is known $[81,83,84]$. Special care is taken to estimate the effect of having a fixed fiducial cosmology for calculating distances and shape of the template for the twopoint statistics. In detail, we measure the range of the differences between true and recovered values obtained by fitting to mocks where the true and fiducial cosmologies do not match. The distribution of cosmologies spanned by the mocks acts as a prior on "allowed cosmologies." All galaxy and quasar tracers used both blind and nonblind mocks to assess their modeling systematic errors. Variations of the Halo Occupation Distribution parametrizations are also taken into account. For the BOSS Galaxy, ELG, and LRG samples, the modeling systematic error is further reduced by scaling the $\sigma_{8}$ value according to the isotropic dilation factor measured independently in the data and in each set of mocks (see Appendix A). For the quasar sample, the redshift determination is an order of magnitude less precise than for the galaxies and requires special modeling. The systematic effect of redshift errors on the two-point statistics is estimated using the N-body mocks [84] and is comparable in size to the systematic errors in modeling.

Observational effects are studied using approximate mocks that are modified to account for the observational conditions $[78,79]$. This includes the dependence of the spectroscopic success rate on the signal-to-noise ratio of the spectra, the treatment of fiber collisions, and the variations of the density of targets for different photometric conditions in the imaging data. For the ELGs and quasars, fiber collisions are taken into account at the model level, and their effect is reduced.

More details about the sets of mocks used to estimate these errors are presented in the papers describing the mocks and the papers describing the individual measurements [68-74]. In summary, for the LRG full-shape analysis, the overall systematic errors amount to about $40 \%$ to $60 \%$ of the statistical error depending on the parameters. The systematic errors for the ELG measurement reaches the same level, although with different sources of systematic effects. For the quasars, the systematic errors are at the level of $30 \%$ of the statistical error for all parameters.
Several tests for systematic errors were performed for the Ly $\alpha$ BAO studies, such as tests on mock spectra, modeling of the broadband signal in the correlation function, and assessment of metal and sky contributions to the Ly $\alpha$ transmission estimates. The central values of the $D_{M} / r_{d}$ and $D_{H} / r_{d}$ estimates did not change significantly during these tests, and no additional systematic errors were included in the reported $\mathrm{BAO}$ results. To account for the somewhat non-Gaussian errors on $D_{M} / r_{d}$ and $D_{H} / r_{d}$, we generated 1000 realizations to estimate the translation of the $\Delta \chi^{2}$ from each measurement in the parameter space to confidence intervals on the BAO parameters. The BAO measurements reported in Table III include this correction.

\section{Summary of SDSS likelihoods}

The final $D_{M}(z) / r_{d}, D_{H}(z) / r_{d}$, and $f \sigma_{8}(z)$ measurements cover eight distinct redshift intervals. The systematic errors and consensus estimates are assessed in the studies that report the final measurements and incorporated directly into the covariance matrices used in this study. Covariances between the two BOSS galaxy measurements are propagated to this study through the same covariance matrix reported by Alam et al. [57].

We find that the expected statistical correlation between clustering measurements derived from the eBOSS samples is negligibly small, and we thus include no covariance between them in our cosmological analyses. This decision for the covariance between the quasar clustering measurements, the Ly $\alpha$ autocorrelation measurements, and the $\operatorname{Ly} \alpha$ quasar cross-correlation measurements was justified using mock catalogs that demonstrated negligible correlation. For the galaxy and quasar samples, the correlation within the overlapping volume can be estimated as

$$
C_{\mathrm{o}}=\frac{P_{1} P_{2}}{\left(P_{1}+1 / n_{1}\right)\left(P_{2}+1 / n_{2}\right)},
$$

where $P$ represents the power-spectrum amplitude and $n$ is the number density. We use the effective $P$ value in Ref. [76] and determine an effective $1 / n$ value based on the effective volume. For both the correlation between the quasars and the ELGs and between the quasars and the LRGs, we find $C_{\mathrm{o}}$ is less than 0.1 , implying any correlation with the quasar sample is negligible. Within their overlapped volume, the expected correlation between the ELGs and LRGs is higher, as each sample has a peak $n P>1$. However, over the full $0.6<z<1.0$ overlap range, we find $C_{\mathrm{o}}=0.24$. Accounting for the fact that the ELG footprint is significantly smaller than the LRG footprint again reduces the expected correlation to less than 0.1 .

Upon final acceptance for publication, the final likelihoods for the MGS, BOSS galaxy, and eBOSS measurements will all be found on the public SDSS svn repository [147] and in the Github repository [148]. The full likelihood is reported for BAO-only studies in the MGS, ELG, 
and Ly $\alpha$ forest samples. The BAO-only results for the BOSS galaxy, eBOSS LRG, and eBOSS quasar samples are recorded as a covariance matrix. We refer to the combination of these measurements as the "BAO" measurements throughout the paper. The combined fits for $\mathrm{BAO}, \mathrm{AP}$, and RSD results are recorded as a full likelihood for the MGS and ELG samples, while the results for the BOSS galaxy, eBOSS LRG, and eBOSS quasar samples are recorded in a single covariance matrix. We refer to these data samples as the "RSD" samples when no information from reconstruction is used and the likelihoods are collapsed to a single dimension on $f \sigma_{8}$. We refer to the full analyses of reconstructed $\mathrm{BAO}$ and full-shape AP + RSD fitting as the "SDSS" sample. In all cases, the likelihoods include both statistical and systematic errors.

\section{B. CMB, SNe, and WL measurements}

The BAO measurements from the four generations of SDSS are complemented by relative distance measurements from SNe Ia. The SDSS RSD measurements are complemented by WL measurements from $\mathrm{CMB}$ and recent imaging programs. CMB anisotropies from allsky, space-based surveys are used throughout to provide a baseline of high-redshift, cosmological measurements. Finally, we compare the local value of the Hubble expansion parameter derived from various combinations of $\mathrm{CMB}$, $\mathrm{BAO}, \mathrm{SNe} \mathrm{Ia}$, and $\mathrm{BBN}$ to the most recent results using local measurements. Neither the BBN nor the $H_{0}$ estimates are directly used in any other cosmological fitting and are not discussed any further in this section, although the BBN constraints on $\omega_{b}$ are used to inform priors in several growth measurements. In the remainder of this section, we discuss the results from the $\mathrm{CMB}, \mathrm{SNe} \mathrm{Ia}$, and WL studies that we use to assess progress in building the cosmological model.

The WMAP satellite launched on June 30, 2001 and ceased scientific operations on August 19, 2010. The cosmological measurements based only on the final WMAP sample provide constraints of $\Omega_{c} h^{2}=0.1138 \pm$ 0.0045 and $\Omega_{b} h^{2}=0.02264 \pm 0.00050$ in a flat $\Lambda \mathrm{CDM}$ model [16]. The Planck satellite [149] operated from 2009-2013 to measure CMB temperature and polarization anisotropies to scales as small as $5^{\prime}$. These measurements allow very precise constraints on the matter content and early expansion history of the Universe, especially in the limit of a $\Lambda \mathrm{CDM}$ cosmology. An analysis under the assumption of a flat $\Lambda \mathrm{CDM}$ model using only Planck temperature and polarization data leads to constraints $\Omega_{c} h^{2}=0.120 \pm 0.001, \Omega_{b} h^{2}=0.0224 \pm 0.0001$, $n_{s}=0.965 \pm 0.004$, and $\tau=0.054 \pm 0.007$ [46]. As the latest generation of $\mathrm{CMB}$ experiment, Planck therefore provides a factor of 4.5 improvement over WMAP on the precision of the dark matter density and a factor of 5 improvement on the precision of the baryonic matter density. When computing constraints using the baseline
Planck measurements, denoted CMB T\&P throughout, we use the PLIK likelihoods for the TT, TE, EE, and lowE power spectra [150]. The data cover multipoles in the range $30 \leq \ell \leq 2508$ for the TT power spectrum and $30 \leq$ $\ell \leq 1996$ for the power spectra that include polarization. When including additional lensing data from Planck denoted "CMB lens," we use the likelihoods from the Planck Collaboration [36] computed over lensing multipoles $8 \leq \ell \leq 400$. When using temperature, polarization, and lensing data together, we refer to the sample simply as "Planck." The full likelihoods for Planck and WMAP measurements are found in the Planck public release of 2018 Cosmological parameters and Monte Carlo (MC) chains [151] and the WMAP 2013 public release, [152] respectively.

At the time that eBOSS began observations, the leading $\mathrm{SNe}$ Ia cosmology studies stemmed from the "joint lightcurve analysis" (JLA) sample. These $740 \mathrm{SNe}$ Ia light curves were taken from low-redshift surveys [21,22], the SDSS-II Supernova Survey $[19,153]$, the Supernova Legacy Survey [23-25], and high-redshift space-based observations with the Hubble Space Telescope [18]. A major effort in the analysis focused on reducing systematic uncertainties in the photometric calibration of the SNLS and SDSS surveys. For a flat $\Lambda$ CDM cosmology using only the $\mathrm{SNe}$ from this sample, the constraints on the matter content of the local universe were found to be $\Omega_{m}=0.295 \pm 0.034$, including systematic errors [113]. More recently, the "Pantheon sample" of 1,048 SNe Ia was used in a comprehensive cosmology analysis. The Pantheon sample includes the full set of spectroscopically confirmed SNe Ia from PanStarrs [154], low redshift SNe Ia [17,21,22,155-157], the SDSS and SNLS SNe Ia samples, and a sample of SNe Ia from the Hubble Space Telescope (HST) $[18,158-161]$. While the increase in sample size since the JLA analysis is significant, the largest improvement in precision results from new cross-calibration of all ground-based measurements to the PanStarrs photometric system. Using only this SN sample with the systematic uncertainties leads to a constraint $\Omega_{m}=0.298 \pm 0.022$ in a flat $\Lambda$ CDM model [114]. Within the basis of a flat $\Lambda \mathrm{CDM}$, the Pantheon sample therefore offers a factor of 1.5 improvement in precision over the JLA sample. Systematic errors are still significant and dominated largely by photometric uncertainties of each sample, the calibration uncertainties of the light-curve model, and the assumption of no redshift dependence of $M_{B}$. The statistical and systematic uncertainties are captured in a covariance matrix with an element for each supernova following the methodology of Conley et al. [24]. The statistical component of the uncertainties contributes only to the diagonal elements, while the off-diagonal elements are dominated by systematic errors arising from common uncertainties in bandpass and zero-point calibration. We primarily use measurements of individual SNe Ia from the Pantheon sample in making 
cosmological constraints and refer to this as the "SN" sample. The covariance matrix for both the JLA sample and the Pantheon sample can be found at the Barbara A. Mikulski Archive for Space Telescopes (MAST) [162] and are included with the COSMOMC installation.

Several recent programs (e.g., Refs. [37,38,42-44]) have reported cosmology constraints from measurements of cosmic shear. Because we are not able to account for covariances between these results due to shared systematic errors, we do not attempt an analysis on the combined weak lensing results. Instead, as an example of how weak lensing data impact cosmological constraints, we focus here on the results from the DES conducted with the Dark Energy Camera [140]. DES released an analysis of cosmic shear using the first year of data covering an area exceeding $1000 \mathrm{deg}^{2}$ with more than 20 million galaxy shape measurements. Tomographic cosmic shear measurements were performed after assigning source galaxies to redshift bins spanning the intervals $0.20<z<0.43,0.43<z<0.63$, $0.63<z<0.90$, and $0.90<z<1.30$. The data are used under an assumption of a $\Lambda \mathrm{CDM}$ model to constrain the combination of $\Omega_{m}$ and $\sigma_{8}$ represented by $S_{8}=0.782 \pm$ 0.027 at $68 \%$ confidence [163]. As in the DES analysis, we only use scales in the cosmic shear correlation functions that are expected to have contributions from baryonic effects of less than $2 \%$. These studies are denoted "WL" in Sec. V. In addition to cosmic shear measurements, we use the $3 \times 2$ pt DES Year 1 results in the analysis presented in Secs. VI and VII, where the additional correlation functions are computed from galaxy clustering and galaxy-galaxy lensing. Following Krause et al. [164], we only use information from the correlation function on comoving scales larger than $8 h^{-1} \mathrm{Mpc}$ for the galaxy clustering measurements and $12 h^{-1} \mathrm{Mpc}$ for the galaxygalaxy lensing. We use the COSMOMC implementation of the DES likelihood [165,166] with covariance matrix, power spectra measurements, and nuisance parameters in agreement with Troxel et al. [163], Krause et al. [164], and Abbott et al. [41]. The combined $3 \times 2$ pt sample is referenced simply as "DES."

\section{IMPLICATIONS OF EXPANSION HISTORY MEASUREMENTS}

In this section, we discuss measurements of the background expansion history, with an emphasis on the BAO measurements from SDSS. We use the Planck temperature and polarization data (CMB T\&P), the SN data from Pantheon, and the BAO data from SDSS. The BAO data, summarized in the BAO-only section of Table III, include measurements from galaxy, quasar, and Ly $\alpha$ forest samples. It is this wide redshift range that enables the tight constraints on cosmological parameters presented in this section.

TABLE IV. Marginalized values and 68\% confidence limits in $\Lambda$ CDM and one-parameter extensions using only expansion history and CMB temperature and polarization measurements.

\begin{tabular}{|c|c|c|c|c|c|c|}
\hline & & $\Omega_{\mathrm{DE}}$ & $H_{0}[\mathrm{~km} / \mathrm{s} / \mathrm{Mpc}]$ & $\Omega_{k}$ & $w$ & $\Sigma_{m_{\nu}}[\mathrm{eV}]^{\mathrm{a}}$ \\
\hline \multirow[t]{5}{*}{$\Lambda \mathrm{CDM}$} & $\mathrm{BAO}$ & $0.701_{-0.015}^{+0.017}$ & $\ldots{ }^{b}$ & $\cdots$ & $\cdots$ & $\cdots$ \\
\hline & CMB T\&P & $0.6836 \pm 0.0084$ & $67.29 \pm 0.61$ & $\cdots$ & $\cdots$ & $\cdots$ \\
\hline & $\mathrm{CMB} \mathrm{T} \& \mathrm{P}+\mathrm{BAO}$ & $0.6881 \pm 0.0058$ & $67.60 \pm 0.43$ & $\cdots$ & $\cdots$ & $\cdots$ \\
\hline & $\mathrm{CMB} \mathrm{T} \& \mathrm{P}+\mathrm{SN}$ & $0.6856 \pm 0.0078$ & $67.43 \pm 0.57$ & $\cdots$ & $\cdots$ & $\cdots$ \\
\hline & $\mathrm{CMB} \mathrm{T} \& \mathrm{P}+\mathrm{BAO}+\mathrm{SN}$ & $0.6890 \pm 0.0057$ & $67.67 \pm 0.43$ & $\ldots$ & $\cdots$ & $\cdots$ \\
\hline \multirow[t]{5}{*}{$\mathrm{o} \Lambda \mathrm{CDM}$} & $\mathrm{BAO}$ & $0.636_{-0.070}^{+0.085}$ & $\cdots$ & $0.079_{-0.10}^{+0.083}$ & $\cdots$ & $\cdots$ \\
\hline & CMB T\&P & $0.561_{-0.041}^{+0.050}$ & $54.5_{-3.9}^{+3.3}$ & $-0.044_{-0.014}^{+0.019}$ & $\cdots$ & $\cdots$ \\
\hline & CMB T \& P + BAO & $0.6884 \pm 0.0059$ & $67.62 \pm 0.62$ & $0.0000 \pm 0.0018$ & $\cdots$ & $\cdots$ \\
\hline & CMB T \& P + SN & $0.670 \pm 0.017$ & $65.2 \pm 2.2$ & $-0.0061_{-0.0054}^{+0.0062}$ & $\cdots$ & $\cdots$ \\
\hline & $\mathrm{CMB} \mathrm{T} \& \mathrm{P}+\mathrm{BAO}+\mathrm{SN}$ & $0.6891 \pm 0.0057$ & $67.67 \pm 0.60$ & $0.0000 \pm 0.0018$ & $\ldots$ & $\cdots$ \\
\hline \multirow[t]{5}{*}{ wCDM } & $\mathrm{BAO}$ & $0.728_{-0.038}^{+0.017}$ & $\ldots$ & $\ldots$ & $-0.69 \pm 0.15$ & $\cdots$ \\
\hline & CMB T\&P & $0.801_{-0.022}^{+0.057}$ & $\ldots^{c}$ & $\cdots$ & $-1.58_{-0.35}^{+0.16}$ & $\cdots$ \\
\hline & $\mathrm{CMB} \mathrm{T} \& \mathrm{P}+\mathrm{BAO}$ & $0.695 \pm 0.013$ & $68.5_{-1.6}^{+1.3}$ & $\cdots$ & $-1.035_{-0.052}^{+0.062}$ & $\cdots$ \\
\hline & $\mathrm{CMB} \mathrm{T} \& \mathrm{P}+\mathrm{SN}$ & $0.692 \pm 0.010$ & $68.3 \pm 1.1$ & $\cdots$ & $-1.035 \pm 0.037$ & $\cdots$ \\
\hline & $\mathrm{CMB} \mathrm{T} \& \mathrm{P}+\mathrm{BAO}+\mathrm{SN}$ & $0.6929 \pm 0.0076$ & $68.23 \pm 0.83$ & $\cdots$ & $-1.027 \pm 0.033$ & $\ldots$ \\
\hline \multirow[t]{4}{*}{$\nu \Lambda \mathrm{CDM}$} & CMB T\&P & $0.680_{-0.0087}^{+0.016}$ & $67.0_{-0.67}^{+1.2}$ & $\cdots$ & $\ldots$ & $<0.268(95 \%)$ \\
\hline & $\mathrm{CMB} \mathrm{T} \& \mathrm{P}+\mathrm{BAO}$ & $0.6892 \pm 0.0065$ & $67.72 \pm 0.50$ & $\cdots$ & $\cdots$ & $<0.129(95 \%)$ \\
\hline & CMB T \& P + SN & $0.686_{-0.0083}^{+0.011}$ & $67.47_{-0.65}^{+0.83}$ & $\cdots$ & $\cdots$ & $<0.174(95 \%)$ \\
\hline & $\mathrm{CMB} \mathrm{T} \& \mathrm{P}+\mathrm{BAO}+\mathrm{SN}$ & $0.6898_{-0.0056}^{+0.0065}$ & $67.76_{-0.44}^{+0.49}$ & $\cdots$ & $\cdots$ & $<0.124(95 \%)$ \\
\hline
\end{tabular}

\footnotetext{
${ }^{\mathrm{a}}$ The reported $\sum m_{\nu}$ values correspond to the $95 \%$ upper limits.

${ }^{\mathrm{b}} \mathrm{BAO}$ measure the dimensionless quantity $r_{d} H_{0} / c$ and therefore can only provide constraints on $H_{0}$ when combined with other probes.

${ }^{\mathrm{c}}$ The constraints of CMB T\&P in the $w \mathrm{CDM}$ model are affected by the $H_{0}$ prior of $H_{0}<100 \mathrm{~km} / \mathrm{s} / \mathrm{Mpc}$, so no entry is provided.
} 


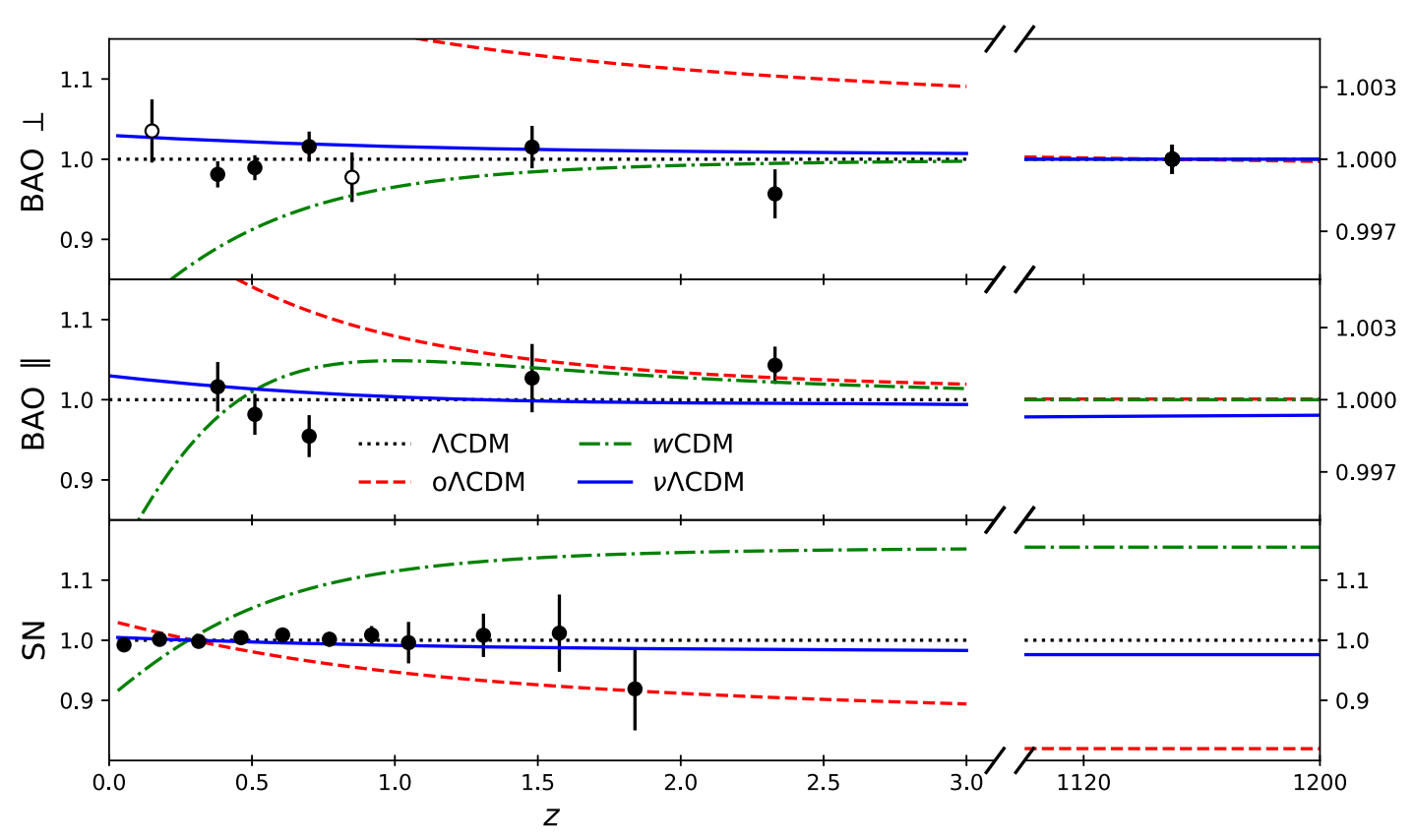

FIG. 2. Demonstration of BAO, SN, and CMB constraining power as a function of redshift. To construct alternative models, we have fixed to their best-fit $\Lambda \mathrm{CDM}$ values the quantities that are best measured by the CMB: $\Omega_{b} h^{2}, \Omega_{c} h^{2}$, and the angular acoustic scale $D_{M}(z=1150) / r_{d}$. Because the sound horizon at decoupling is a function of $\Omega_{b} h^{2}, \Omega_{c} h^{2}$, and $N_{\text {eff }}$ only, the models have the same value of $r_{d}=147.16 \mathrm{Mpc}$. Top: the Hubble diagram residuals of BAO $D_{M}(z)$ measurements, presented as the ratio of the measured value of $D_{M}(z) / r_{d}$ relative to the prediction for that value based on the best-fit $\Lambda$ CDM model from CMB alone. $D_{V}(z)$ measurements are shown as open circles. We display the CMB determination of the angular position of the acoustic peak as a measurement of transverse BAO, and we split the redshift scale to include this data point. Center: the Hubble diagram residuals of BAO $D_{H}(z)=c / H(z)$ measurements, normalized in the same manner as the $D_{M}(z)$ measurements. Bottom: the Hubble diagram residuals of SNe Ia measurements, with relative normalization of the luminosity distance estimates. In order to increase the signal to noise, the supernovae data were binned into 11 bins between redshifts 0.1 and 2.5. Spacing was chosen to maintain a relatively constant signal-to-noise ratio. Since the distance modulus varies significantly across the bin at low redshift, we have averaged the signal by averaging the inverse covariance weighted deviations from the $\Lambda$ CDM model after the absolute normalization has been fitted. The covariance matrix was taken from the Pantheon dataset. In each case, the residuals are computed relative to the best-fit $\Lambda C D M$ model from $C M B$ alone. The curves represent the difference between the $\Lambda$ CDM model and single-parameter extensions allowed by the CMB data. The o $\Lambda$ CDM model favored by Planck $\left(\Omega_{k}=-0.044\right)$ is presented in dashed red lines, the $w$ CDM model favored by Planck $(w=-1.585)$ is presented in dot-dashed green lines, and a $\Lambda$ CDM model with nonzero neutrino mass is presented in solid blue lines. The model with massive neutrinos assumes a summed mass equal to $0.268 \mathrm{eV}$, corresponding to the Planck $95 \%$ upper limit.

We start in Sec. IV A with a discussion on the role of BAO and $\mathrm{SN}$ measurements on single-parameter extensions to the $\Lambda \mathrm{CDM}$. By adding measurements of the expansion history, we show that we can break parameter degeneracies present in the $\mathrm{CMB}$ results, leaving combined fits that are always consistent with a flat $\Lambda \mathrm{CDM}$ model. The combined probes also offer some of the most competitive constraints on neutrino mass without adding any information from growth of structure. In Sec. IV B, we show that the BAO data enable estimates of $H_{0}$ that are robust against the assumption of cosmological model and estimates that are independent of CMB anisotropies altogether.

\section{A. Impact of BAO measurements on models for single-parameter extensions to $\Lambda \mathrm{CDM}$}

We first report the results in the simplest cosmology, that of a spatially flat universe where dark energy can be explained by a cosmological constant $(\Lambda \mathrm{CDM})$. As shown in Table IV, CMB data alone are sufficient to constrain the dark energy density parameter to roughly $1 \%$ precision. Adding the $\mathrm{BAO}$ and $\mathrm{SN}$ data improves this constraint by a factor of 1.5 for this simplest model of the expansion history.

Figure 2 shows the residuals of the $\mathrm{BAO}$ and $\mathrm{SNe} \mathrm{Ia}$ distances with respect to the $\Lambda$ CDM model favored by the Planck temperature and polarization data. The BAO and $\mathrm{SNe}$ Ia data have a combined $\chi^{2} / \mathrm{DoF}<1$ with respect to this model, indicating very good agreement. In order to highlight how BAO and SNe Ia data complement the CMB results in models with a single-parameter extension to $\Lambda \mathrm{CDM}$, in Fig. 2, we also show the prediction for three models that are allowed by Planck but are ruled out by measurements of the low-redshift expansion history: an o $\Lambda$ CDM model with the Planck-favored value of $\Omega_{k}=$ -0.044 (dashed red), a wCDM model with the Planckfavored value of $w=-1.585$ (dot-dashed green), and a $\Lambda \mathrm{CDM}$ model with the Planck $95 \%$ upper limits on the 

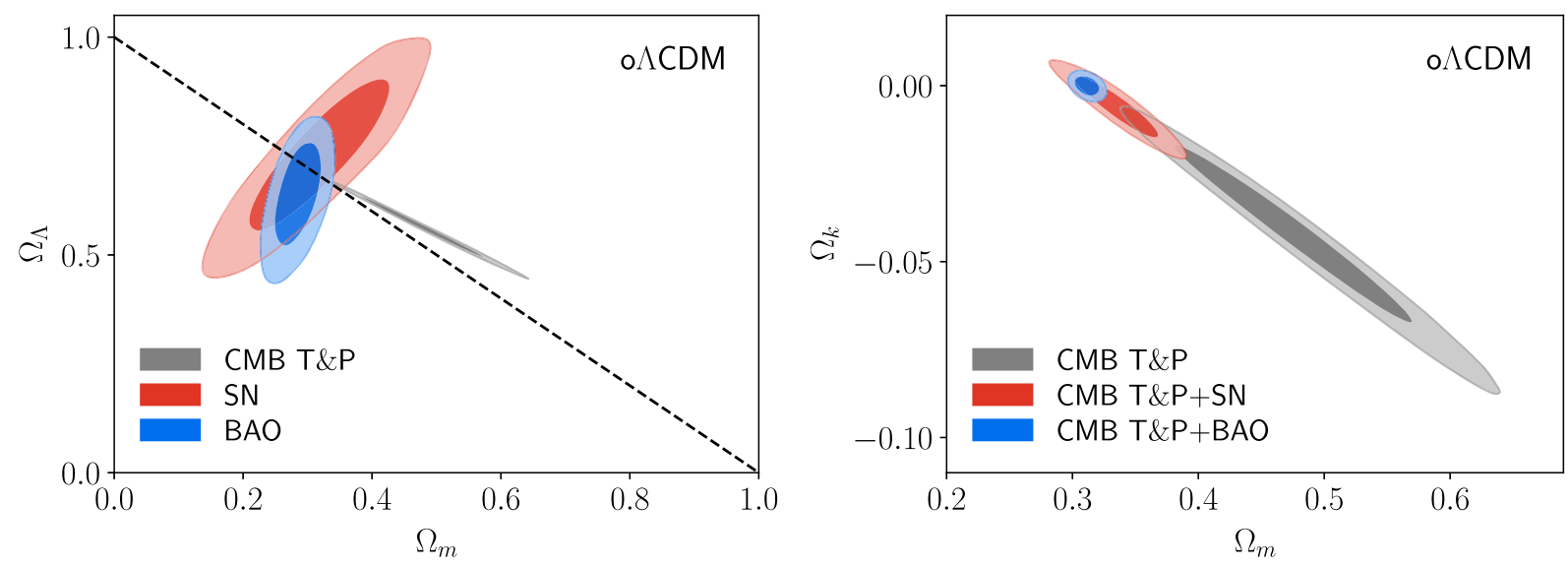

FIG. 3. Cosmological constraints under the assumption of a model with a $w=-1$ cosmological constant with free curvature (o $\Lambda$ CDM, as in Table IV). Left: $68 \%$ and $95 \%$ constraints on $\Omega_{m}-\Omega_{\Lambda}$ from the Planck CMB temperature and polarization data (gray), Pantheon SNe Ia sample (red) and SDSS BAO-only measurements (blue). The dashed line represents a model with zero curvature. Right: the $\Omega_{m}-\Omega_{k}$ constraints for the combination of CMB (gray), CMB $+\mathrm{SN}$ (red), and CMB $+\mathrm{BAO}$ (blue).

sum of the neutrino masses of $\sum m_{\nu}=0.268 \mathrm{eV}$ (solid blue). ${ }^{1}$

In the next subsections, we present in detail how BAO and $\mathrm{SNe}$ Ia can break strong degeneracies present in the CMB data when studying single-parameter extensions to the $\Lambda \mathrm{CDM}$ model.

\section{Expansion history and curvature}

The Planck temperature and polarization data alone offer strong constraints within the $\mathrm{o} \Lambda \mathrm{CDM}$ model, but with degenerate posteriors as shown in both panels of Fig. 3. The consequences of these degeneracies are quantified in Table IV, where the uncertainty on $\Omega_{\Lambda}$ in this model is five times larger than in a flat $\Lambda \mathrm{CDM}$ model. The preference for a closed universe, with a significance slightly above $95 \%$ confidence, is discussed in detail in Refs. [46,167]. As shown in Fig. 2, the predictions from the closed universe favored by the CMB (dashed red lines) are disallowed at high confidence by both the BAO and the SN data.

In an $\mathrm{o} \Lambda \mathrm{CDM}$ model, BAO measurements at different redshifts constrain different combinations of $\left(\Omega_{m}, \Omega_{k}\right.$, and $\left.r_{d} H_{0} / c\right)$. When we combine BAO results over a wide redshift range, we are able to break internal degeneracies and provide independent constraints on these parameters (e.g., Ref. [107]). Table IV and the left panel of Fig. 3 show that BAO measurements alone lead to $\Omega_{\Lambda}=0.636_{-0.070}^{+0.085}$, an approximately $8 \sigma$ confidence detection of a cosmological constant without any information from the CMB or SNe Ia data. The SNe Ia data alone also favor a flat geometry but are not as constraining as BAO. Using only $\mathrm{SNe}$ Ia leads to a detection of $\Omega_{\Lambda}=0.73 \pm 0.11$.

\footnotetext{
${ }^{1}$ The slight change in neutrino mass compared to the Planck analysis is due to our use of an updated version of COSMOMC.
}

The right panel of Fig. 3 demonstrates that including either BAO or SN data reduces the parameter degeneracies in the CMB data. The $\Omega_{\mathrm{DE}}$ results in the CMB T \& P + BAO entries in Table IV are almost the same in $\Lambda \mathrm{CDM}$ and $\mathrm{o} \Lambda \mathrm{CDM}$ models. The combination of BAO and CMB data favors a flat universe with $\Omega_{k}=-0.0000 \pm 0.0018$.

\section{Expansion history and dark energy}

We next consider a flat $w \mathrm{CDM}$ model, with an extra free parameter $w$ to describe the equation of state of dark energy. As with the o $\Lambda C D M$ model, the left panel of Fig. 4 shows that the $\mathrm{CMB}$ temperature and polarization data leave strong degeneracies between the $w$ and energy density parameters that determine the expansion history. Table IV shows that the constraints on $\Omega_{\mathrm{DE}}$ are again degraded by a factor of about 5 with respect to the constraints in a $\Lambda$ CDM model, with a shift in the central value that is opposite in direction to the shift in the o $\Lambda \mathrm{CDM}$ model. The models with very negative values of $w$ favored by CMB (dotdashed green lines in Fig. 2) are inconsistent with both the $\mathrm{BAO}$ and the SN data.

As shown in the left panel of Fig. 4, the Planck $\Lambda$ CDM values $\left(w=-1\right.$ and $\left.\Omega_{m}=0.3164\right)$ lie within the 95\% confidence intervals of both the BAO data alone and the SN data alone. The BAO data alone are able to constrain the matter density without a strong degeneracy with $w$. Even though the SNe Ia contours have a strong degeneracy in $w-\Omega_{m}$, the contours are perpendicular to the degeneracy direction of the CMB contours, so the $\mathrm{CMB} \mathrm{T} \& \mathrm{P}+\mathrm{SN}$ combination results in very tight constraints on the $w \mathrm{CDM}$ model. Each of the three combinations, $\mathrm{CMB} \mathrm{T} \& \mathrm{P}+\mathrm{BAO}, \mathrm{CMB} \mathrm{T} \& \mathrm{P}+\mathrm{SN}$, and even $\mathrm{BAO}+\mathrm{SN}$ favor a model with a cosmological constant. As shown in Table IV, the combination of all three datasets results in a measurement of the equation of state of dark 

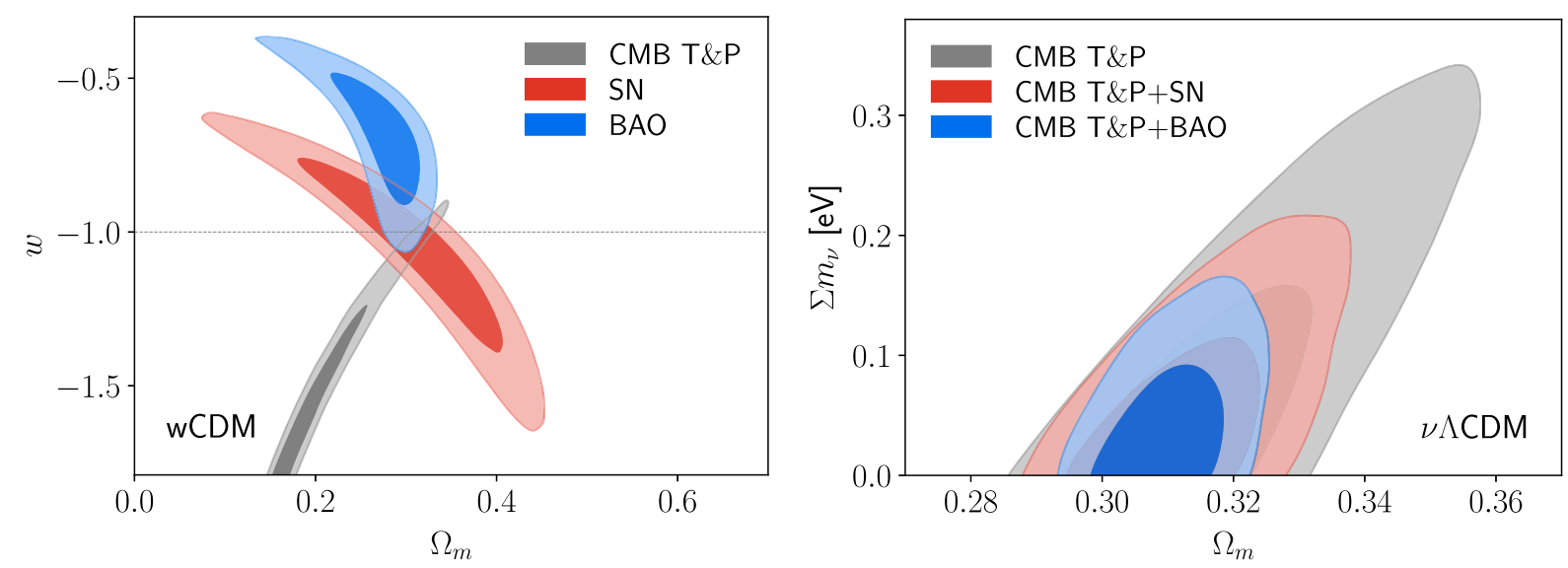

FIG. 4. Constraints on the $w C D M$ and $\nu \Lambda C D M$ models, as in Table IV. Left: $w-\Omega_{m}$ constraints under the assumption of a flat $w C D M$ cosmology from the Planck CMB temperature and polarization data (gray), Pantheon SNe Ia sample (red) and SDSS BAO-only measurements (blue). Right: $\sum m_{\nu}-\Omega_{m}$ constraints under the assumption of a flat $\Lambda$ CDM cosmology where the summed neutrino mass is allowed as a free parameter, for the combination of $\mathrm{CMB}$ (gray), $\mathrm{CMB}+\mathrm{SN}$ (red), and $\mathrm{CMB}+\mathrm{BAO}$ (blue).

energy of $w=-1.027 \pm 0.033$, consistent with a cosmological constant.

\section{Expansion history and neutrino masses}

We now turn our attention to a $\nu \Lambda \mathrm{CDM}$ model where the sum of the neutrino masses is considered a free parameter. As shown in the right panel of Fig. 4 and in Table IV, the Planck temperature and polarization data offer a $95 \%$ upper bound on the summed neutrino mass of $268 \mathrm{meV}$. Neutrinos lighter than approximately $500 \mathrm{meV}$ are still relativistic at the time of recombination, but they impact $\mathrm{CMB}$ observables by modifying the late-time expansion, in particular, the angular diameter distance to the epoch of recombination $D_{M}\left(z_{\text {rec }}\right)$. Neutrino mass constraints from the CMB are therefore degenerate with other cosmological parameters that modify $D_{M}\left(z_{\text {rec }}\right)$, like $\Omega_{m}$ or $H_{0}$. Late-time measurements of the expansion can break this degeneracy, as shown in blue lines of Fig. 2 and in the right panel of Fig. 4. Adding $\mathrm{BAO}$ or $\mathrm{SN}$ data reduces the upper bound on the sum of neutrino masses by factors of 2 and 1.5, respectively. Combining the three datasets, we obtain a 95\% upper limit of $124 \mathrm{meV}$.

In this subsection, we have shown that measurements of the expansion history are very complementary to measurements of CMB temperature and polarization anisotropies. As shown in Fig. 2, both BAO and SNe Ia are able to constrain single-parameter extensions to $\Lambda \mathrm{CDM}$ that cannot be constrained by CMB alone. As shown in Table IV, adding $\mathrm{BAO}$ to the $\mathrm{CMB}$ data reduces the uncertainty on $\Omega_{\mathrm{DE}}$ in $\mathrm{o} \Lambda \mathrm{CDM}$ models by a factor of 8 , and it excludes models with curvature that would otherwise be favored by the CMB. Similarly, adding SN and BAO to the CMB reduces the uncertainty on $\Omega_{\mathrm{DE}}$ in $w \mathrm{CDM}$ models by more than a factor of 5, and it excludes models with $w<-1$ favored by the CMB. For all models discussed, the combination of all three probes results in a percent measurement of $\Omega_{\mathrm{DE}}$, consistent with $\Omega_{\mathrm{DE}}=0.69$.

\section{B. BAO and the $\boldsymbol{H}_{\mathbf{0}}$ tension}

The present-day expansion rate, $H_{0}$, is one of the basic parameters in the cosmological model because it allows absolute estimates of the age and the current energy content of the Universe. It is one of the three fundamental cosmological parameters that are not dimensionless (the two other being the temperature of the $\mathrm{CMB}$ and the neutrino masses). Moreover, as discussed by $\mathrm{Hu}$ [168] and by Weinberg et al. [104], an accurate measurement of $H_{0}$ would allow a powerful test of dark energy models and tightened constraints on cosmological parameters. However, a statistically significant tension has been demonstrated between direct measurements of $H_{0}$ from the local distance ladder and those estimates of $H_{0}$ inferred from the CMB [169]. This tension has persisted and even increased in significance, despite significant effort to identify possible sources of systematic errors.

Measurements of the Hubble constant come in different flavors, as shown in the compilation of studies presented in the bottom part of Table V. An example of direct measurement, referred to here as the distance ladder, uses parallaxes from local stars and other techniques to calibrate distances to Cepheid variables, which are in turn used for absolute luminosity calibration of $\mathrm{SNe}$ Ia hosted by nearby galaxies (e.g., Ref. [45]). The calibrated luminosity is used to estimate the absolute luminosity distance to a sample of SNe Ia that covers a redshift range sufficient to minimize the effect of peculiar velocities relative to the Hubble flow. Similar efforts include the use of other distance indicators such as the tip of the red giant branch (TRGB) (e.g., Ref. [170]), Tully-Fisher relation in galaxies (TFR) (e.g., Ref. [171]), or gravitational waves from 
TABLE V. Effect of BAO measurements on Hubble constant constraints.

\begin{tabular}{lccc}
\hline \hline Dataset & Cosmological model & $H_{0}\left(\mathrm{~km} \mathrm{~s}^{-1} \mathrm{Mpc}^{-1}\right)$ & Comments \\
\hline CMB T \& P + BAO + SN & ow $w_{a} \mathrm{CDM}$ & $67.91 \pm 0.87$ & Inverse distance ladder \\
BBN + BAO & $\Lambda \mathrm{CDM}$ & $67.33 \pm 0.98$ & No CMB anisotropies \\
CMB T\&P & $\Lambda \mathrm{CDM}$ & $67.28 \pm 0.61$ & Planck 2018 [46] \\
CMB T\&P & $\mathrm{o} \Lambda \mathrm{CDM}$ & $54.5_{-3.3}^{+3.3}$ & Planck 2018 [46] \\
Lensing time delays & $\Lambda \mathrm{CDM}$ & $73.3 \pm 1.8$ & HOLiCOW [173] \\
Distance ladder & $\ldots$ & $74.0 \pm 1.4$ & SHOES [45] \\
GW sirens & $\ldots$ & $70 \pm 10$ & LIGO [172] \\
TRGB & $\ldots$ & $69.6 \pm 1.9$ & LMC anchor [170] \\
TFR & $\ldots$ & $76.2 \pm 4.3$ & Cosmicflows [174] \\
Maser galaxies & $\ldots$ & $73.9 \pm 3.0$ & Megamaser Cosmology Project [175] \\
\hline \hline
\end{tabular}

neutron star-neutron star mergers (e.g., Ref. [172]). These measurements typically measure higher values of the Hubble constant. For example, Riess et al. [45] perform a study using SNe Ia distances calibrated from 70 long-period Cepheids in the Large Magellanic Cloud. They find $H_{0}=74.03 \pm 1.42 \mathrm{~km} \mathrm{~s}^{-1} \mathrm{Mpc}^{-1}$, including systematic errors.

Other measurements of $H_{0}$ involve data at higher redshift and need to assume a cosmological model to extrapolate the constraints to redshift zero. One example of this indirect measurement is that obtained using time delays in strongly lensed quasars (e.g., Ref. [176]). Other indirect measurements of $H_{0}$ use CMB data under strong assumptions about the model governing the expansion history from the last scattering surface to today. The CMB estimates typically give considerably lower values of the Hubble constant. The final Planck data release, for example, finds $H_{0}=67.36 \pm 0.54 \mathrm{~km} \mathrm{~s}^{-1} \mathrm{Mpc}^{-1}$ [46] when assuming the $\Lambda \mathrm{CDM}$ model.

Explanations for the tension between direct measurements and $\mathrm{CMB}$ estimates range from underestimated systematic errors or modeling of the primordial power spectrum (e.g., Refs. [177-180]), to models for dark energy (e.g., Refs. [181-183]), to unmodeled prerecombination physics that lead to a decreased sound horizon scale (e.g., Refs. [184-189]). See Ref. [190] for a review of possible solutions to the tension.

We provide here two alternative analyses to show how BAO measurements allow estimates of $H_{0}$ that are robust against the strict assumptions of the CMB-only estimates. First, we combine Planck temperature and polarization, $\mathrm{SN}$, and BAO data and allow a very flexible expansion history to demonstrate that the tension in $H_{0}$ estimates is not due to the assumptions of a $\Lambda C D M$ model. Second, we present a measurement of $H_{0}$ that uses BAO and a $\mathrm{BBN}$ prior that is independent of $\mathrm{CMB}$ anisotropies to demonstrate that the tension is not due to systematic errors in the CMB data. We finish this section by presenting the combination of the BAO data with the local distance ladder measurement, and we discuss the low value of $r_{d}$ inferred from this analysis.

\section{1. $H_{0}$ and the inverse distance ladder}

In this subsection, we present a cosmological measurement of $H_{0}$ using a model for expansion history that allows three additional free parameters beyond $\Lambda \mathrm{CDM}$. This approach is often referred as the inverse distance ladder, as it relies on a calibrated distance measure at high redshift that is then extrapolated to $z=0$. Schematically, we use information from the $\mathrm{CMB}$ to calibrate the BAO distances. Those in turn are used to calibrate the absolute luminosity of SNe Ia.

Since the BAO feature follows $D_{H}(z) / r_{d}=c / H(z) / r_{d}$ and $D_{M}(z) / r_{d}$, rather than $H(z)$ directly, this measurement relies on a calibration of the sound horizon $\left(r_{d}\right)$ at the drag epoch to extract the Hubble parameter. Under the implicit assumption of a smooth expansion history, standard prerecombination physics, and a well-measured mean temperature of the CMB, $r_{d}$ only depends on the cold dark matter density $\left(\Omega_{c} h^{2}\right)$ and the baryon density $\left(\Omega_{b} h^{2}\right)$. Thus, $r_{d}$ can be calibrated through constraints arising from the full CMB temperature and polarization likelihoods, with little dependence on the late-time history of the Universe, as demonstrated in Fig. 2 of Ref. [191].

The extrapolation of $H(z)$ measurements from BAO to $z=0$ can be done using a very flexible cosmology because both $\mathrm{BAO}$ and $\mathrm{SNe}$ Ia relative distance measurements constrain the evolving expansion rate. The inclusion of BAO makes the technique robust to the assumed properties of dark energy as was demonstrated in earlier BOSS analyses [192].

We choose an $\mathrm{ow}_{0} w_{a} \mathrm{CDM}$ model to allow for a flexible expansion history of the Universe. Note that CMB alone cannot constrain this model; as shown in Table $\mathrm{V}$, the uncertainties on $H_{0}$ from CMB constraints already increase by a factor of about 6 when we consider only oneparameter extensions, such as models with curvature. The combination of $\mathrm{CMB}, \mathrm{BAO}$, and $\mathrm{SN}$ data, however, is able to provide a very precise measurement of $H_{0}$ even in this flexible model. Our results, presented in Table V and in the left panel of Fig. 5, have an uncertainty better than $1 \mathrm{~km} \mathrm{~s}^{-1} \mathrm{Mpc}^{-1}$ and are consistent with the low value of $H_{0}$ measured by the $\mathrm{CMB}$ under the strict assumption of $\Lambda \mathrm{CDM}$. 

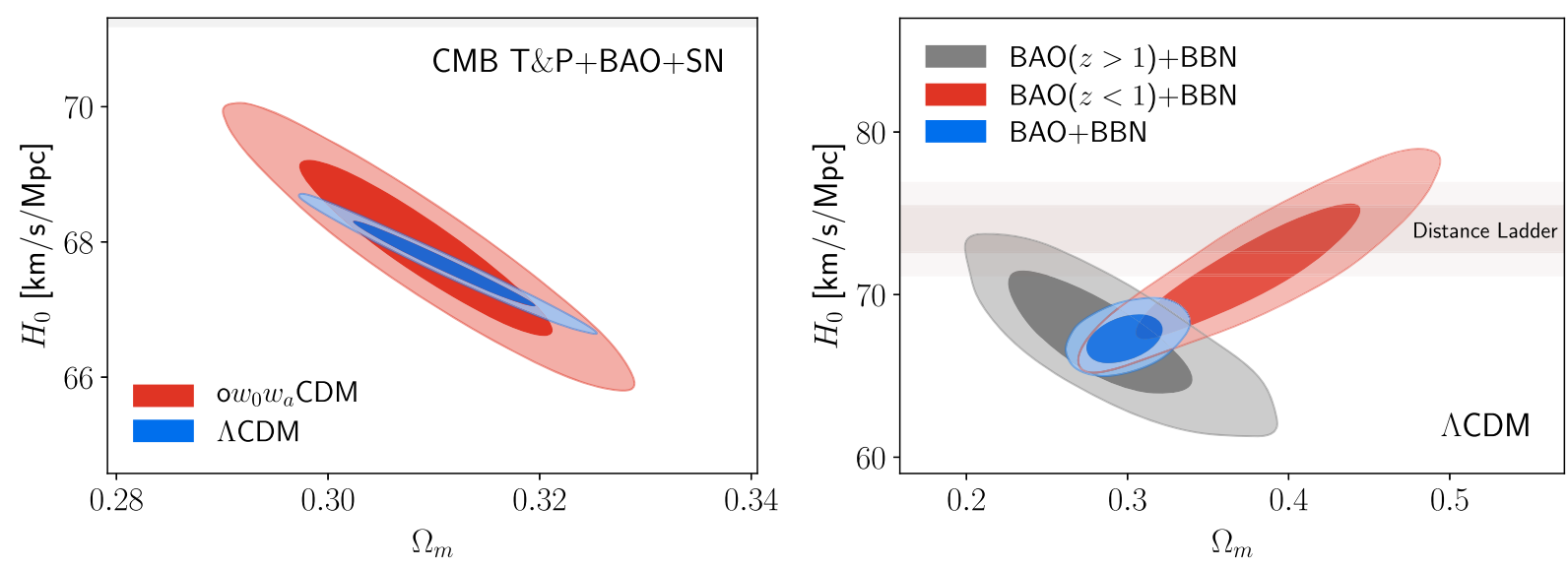

FIG. 5. Left: $H_{0}$ vs $\Omega_{m}$ from the inverse distance ladder $(\mathrm{CMB}+\mathrm{BAO}+\mathrm{SN})$ under two different cosmological models. Right: $H_{0}$ vs $\Omega_{m}$ from the combination of BAO and BBN, in a $\Lambda$ CDM model (blue). The red (gray) contours show the results when using only BAO measurements below (above) $z=1$. The horizontal shaded area shows the $(68 \%, 95 \%)$ measurement of $H_{0}$ from the distance ladder technique (SHOES) [45].

\section{2. $\mathrm{H}_{0}$ independent of $\mathrm{CMB}$ anisotropies}

In the previous subsection, we showed that the value of $H_{0}$ measured by the combination of $\mathrm{CMB}, \mathrm{BAO}$, and $\mathrm{SN}$ data is robust under different models for curvature and dark energy equation of state. In this section, we return to the $\Lambda \mathrm{CDM}$ model and present a measurement of $H_{0}$ that is independent of $\mathrm{CMB}$ anisotropies.

The combination of BAO measurements at different redshifts can provide a precise measurement of the dimensionless quantity $r_{d} H_{0} / c$. To translate constraints on this dimensionless quantity to a measurement of $H_{0}$, we use information on $\omega_{b}$ by including BBN constraints; $\omega_{c}$ and $H_{0}$ are also left free as they can be determined in the fitting by the BAO data. ${ }^{2}$ We use the results of recent highresolution spectroscopic measurements of seven quasar absorption systems that indicate a primordial deuterium abundance $\mathrm{D} / \mathrm{H}=(2.527 \pm 0.030) \times 10^{-5}$ [194]. Using the empirically derived reaction cross section [195], the deuterium abundances imply $\omega_{b}=0.02235 \pm 0.00037$ under an assumption that $N_{\text {eff }}=3.046$. The 68\% confidence interval reflects the combined deuterium abundance and reaction rate uncertainties.

As can be seen in the right panel of Fig. 5, we obtain a tight constraint on $H_{0}$ only when we combine BAO measurements from a wide redshift range. In particular, the line-of-sight BAO measurements above $z=1$ (from quasars and the $\operatorname{Ly} \alpha$ forest) provide measurements of the expansion in the matter-dominated area, and their contours have different degeneracies in the $\left(\Omega_{m}, H_{0}\right)$ plane.

As shown in Table $\mathrm{V}$, the precision on $H_{0}$ when combining $\mathrm{BAO}$ measurements with a $\mathrm{BBN}$ prior is $0.98 \mathrm{~km} \mathrm{~s}^{-1} \mathrm{Mpc}^{-1}$. This result is consistent with the

\footnotetext{
${ }^{2}$ To estimate the radiation density, we also use the absolute CMB temperature measured by FIRAS, $T_{0}=2.7255 \mathrm{~K}$ [193].
}

findings of Refs. [196,197], which used BAO data from SDSS DR12 and DR14, respectively. The central value remains relatively unchanged from the results using $\mathrm{CMB}$, $\mathrm{BAO}$, and $\mathrm{SN}$ data in the $\mathrm{o} w_{0} w_{a} \mathrm{CDM}$ model, providing further evidence that the tension is not due to peculiarities in the CMB anisotropy data.

\section{Sound horizon at drag epoch from low redshifts}

As shown above, the BAO data in combination with information on the baryon density from the early Universe can be used to extrapolate late Universe expansion history to constrain the Hubble constant. The BAO data can also be used to constrain the sound horizon at the drag epoch when combined with local $H_{0}$ measurements (e.g., Ref. [191]).

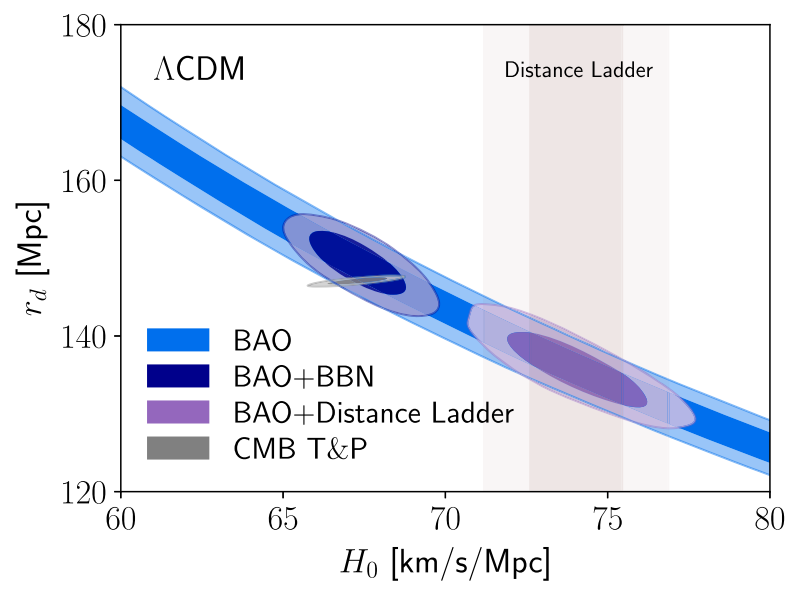

FIG. 6. Cosmological constraints on $H_{0}$ and $r_{d}$ under the assumption of the $\Lambda \mathrm{CDM}$ model using BAO data (blue) in combination with $H_{0}$ distance ladder measurements (purple) and $\mathrm{BBN}$ data (dark blue) in contrast to CMB measurements (gray). The shaded band refers to the $H_{0}$ distance ladder measurement. 
Figure 6 shows the two-dimensional contours of $H_{0}$ and $r_{d}$ for BAO data in combination with different datasets under the assumption of a $\Lambda \mathrm{CDM}$ model. The BAO data alone are completely degenerate in the $H_{0}-r_{d}$ plane; however, this degeneracy can be broken by either local $H_{0}$ measurements, by BBN, or by CMB data. The local $H_{0}$ measurements are clearly in tension with early Universe measurements of the sound horizon. $\mathrm{BAO}$ and $\mathrm{BBN}$ data prefer a value $r_{d}=149.3 \pm 2.7 \mathrm{Mpc}$, in good agreement with the value $r_{d}=147.06 \pm 0.29 \mathrm{Mpc}$ preferred by the $\mathrm{CMB}$ temperature and polarization data alone. These estimates are much larger than the $\mathrm{BAO}$ and distance ladder constraint of $r_{d}=135.8 \pm 3.2 \mathrm{Mpc}$. These constraints on $r_{d}$ can also be translated into limits on the baryon density, yielding $\omega_{b}=0.0310 \pm 0.0023$ for BAO and distance ladder data. In comparison, the $\mathrm{CMB}$ best fit of $\omega_{b}=0.02236 \pm 0.00015$ or the BBN best fit of $\omega_{b}=$ $0.02235 \pm 0.00037$ is much lower.

Finally, dropping the assumption of a $\Lambda \mathrm{CDM}$ model and including SN in our analysis of the distance ladder, we find $r_{d}=135.2 \pm 3.1 \mathrm{Mpc}$ and $\omega_{b}=0.0375_{-0.0085}^{+0.0054}$ in a o $w_{0} w_{a} \mathrm{CDM}$ model. This extended distance ladder measurement shows that the discrepancy between low- and highredshift measurements of the sound horizon is independent of the assumption of the cosmological model. However, we caution that we did not take the correlation between the SN data and the local $H_{0}$ measurement into account for our analysis.

To summarize, the BAO data allow robust, consistent measurements of $H_{0}$ that include freedom in expansion history beyond the strict $\Lambda \mathrm{CDM}$ assumptions in CMBonly estimates. The BAO data allow robust, consistent measurements of $H_{0}$ that are insensitive to the use of CMB anisotropies altogether if using the $\Lambda$ CDM model. In all cases, the central values remain below $H_{0}=68 \mathrm{~km} \mathrm{~s}^{-1} \mathrm{Mpc}^{-1}$, and the uncertainties remain at $1 \mathrm{~km} \mathrm{~s}^{-1} \mathrm{Mpc}^{-1}$ or better.

On the other hand, the Cepheid distance ladder or strong lensing time delays of quasars provide precise estimates of $H_{0}$ that favor larger values of $H_{0}$, or smaller values of $r_{d}$ if being used to calibrate the $\mathrm{BAO}$ scale. Combining their results as independent measurements produces an estimate of $H_{0}=73.7 \pm 1.1 \mathrm{~km} \mathrm{~s}^{-1} \mathrm{Mpc}^{-1}$. This central value differs from those presented in this work by more than four standard deviations whether we use a multiple-parameter model for expansion or the BBN measurements of $\omega_{b}$. The consistency of the results highlights that the " $H_{0}$ tension" cannot be restricted to systematic errors in Planck or to the strict assumptions of the $\Lambda \mathrm{CDM}$ model.

Both the CMB analysis and those presented here are sensitive to the assumption of standard prerecombination physics that sets the scale of $r_{d}$. As summarized by Knox and Millea [190], there have been many attempts to reconcile the $H_{0}$ tension by modifying the value of $r_{d}$, with limited success.

\section{IMPLICATIONS OF GROWTH MEASUREMENTS}

A key development of the BOSS and eBOSS surveys is the advancement of RSD as a tool to make high-precision measurements of structure growth over a wide redshift range. In this section, we assess the impact of those growth measurements on the cosmological model. We first compare the RSD measurements to DES weak lensing and Planck lensing results to complement the CMB temperature and polarization data in dark energy models. To achieve "RSDonly" constraints, we marginalize the dependency of $D_{H}$ and $D_{M}$ out of our "full-shape" measurement and use only the results on the growth, $f \sigma_{8}(z)$. In the second part of this section, we explore the use of growth measurements to constrain matter fluctuations and to test the assumptions of GR in the cosmological model.

\section{A. Impact of RSD measurements on models for single-parameter extensions to $\Lambda \mathrm{CDM}$}

The constraining power of RSD is illustrated in Fig. 7. The low-redshift RSD measurements alone have sensitivity to rule out Einstein-de Sitter $\left(\Omega_{m}=1\right)$ models, while the higher-redshift RSD measurements are sensitive to variations in the dark energy equation of state. We first quantify how these RSD data offer complementary views to the WL data on single-parameter extensions to a $\Lambda \mathrm{CDM}$ cosmology.

\section{Expansion history and curvature}

We begin by exploring the constraints on a model with free curvature $(\mathrm{o} \Lambda \mathrm{CDM})$ using growth measurements combined with the Planck CMB temperature and polarization data. The marginalized $68 \%$ constraints on key cosmological parameters are shown in the top half of Table VI. The two-dimensional contours on $\Omega_{m}$ and $\Omega_{\Lambda}$ are shown in the left panel of Fig. 8. While the Planck CMB data alone favor a model with negative curvature, the combination with all growth measurements (RSD, WL, and CMB lensing) reduces the $\Omega_{k}$ uncertainty by a factor of 4 and leads to a model consistent with zero curvature $\left(\Omega_{k}=-0.0010_{-0.0039}^{+0.0044}\right)$.

As shown in the residual diagram of growth measurements (Fig. 7), the predictions for growth in a free-curvature $\mathrm{o} \Lambda \mathrm{CDM}$ universe have the largest deviations from a $\Lambda \mathrm{CDM}$ prediction as redshift approaches $z=0$. The RSD measurements in this regime are governed largely by the MGS sample, with a precision of $36 \%$ on $f \sigma_{8}$. As a consequence, relative to the CMB-only constraints on curvature, those from the $\mathrm{CMB}+\mathrm{RSD}$ measurements only result in a mild shift with a slightly reduced uncertainty (Table V). The DES WL data, on the other hand, offer an independent measurement of the mass distribution, in particular, using source galaxies over the redshift range $0.20<z<0.43$. While difficult to visualize in a manner similar to the RSD, the WL measurements offer significantly higher-precision estimates 


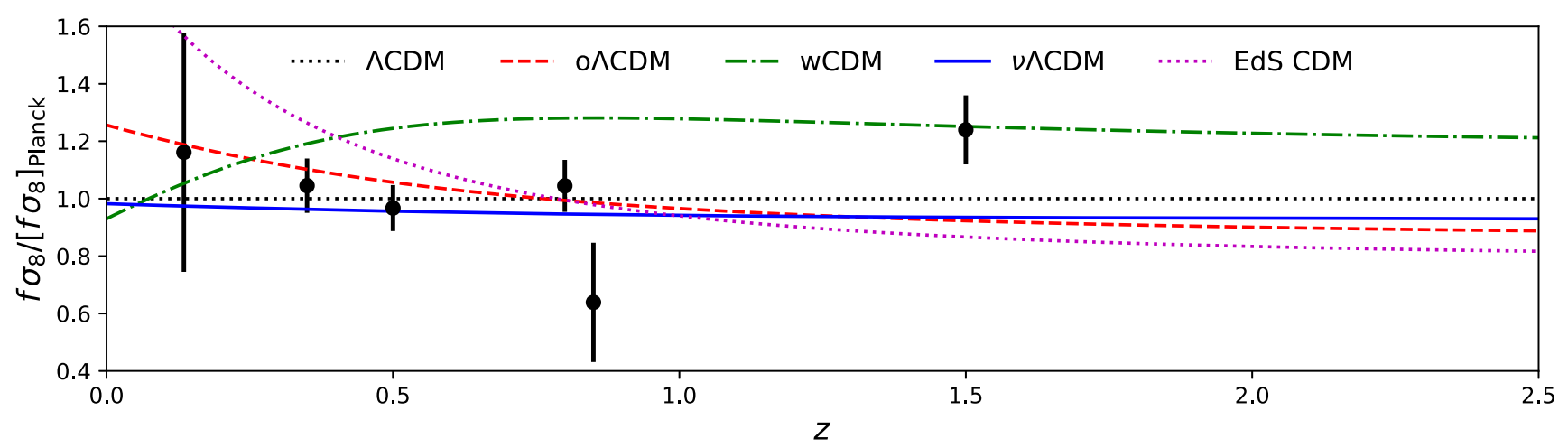

FIG. 7. The SDSS $f \sigma_{8}$ measurements as a function of redshift, normalized by the Planck 2018 best-fit $\Lambda$ CDM model (shown in dotted black). The three colored curves represent the fractional deviations from $\Lambda \mathrm{CDM}$ for an $\mathrm{o} \Lambda \mathrm{CDM}$ model with $\Omega_{k}=-0.044$ (red), a $w \mathrm{CDM}$ model with $w=-1.58$ (green), and a $\nu \Lambda \mathrm{CDM}$ model with $\sum m_{\nu}=0.268 \mathrm{eV}$ (blue). These are the same models as those in Fig. 2. An Einstein-de Sitter model (magenta; $\Omega_{m}=1, \Omega_{\Lambda}=0$, and $\sigma_{8}(z=0)$ matching that of fiducial model) is ruled out at high confidence, further demonstrating the long-standing preference for growth measurements for models with lower matter densities.
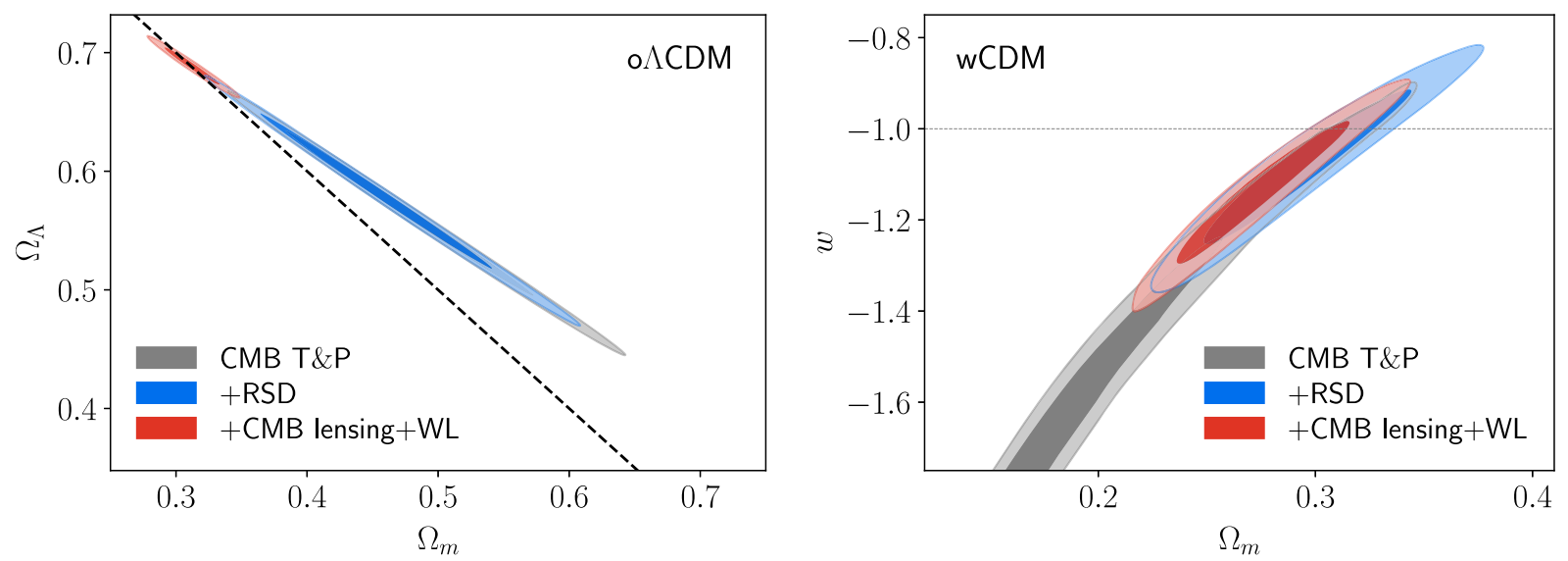

FIG. 8. Constraints from CMB temperature and polarization and growth measurements in one-parameter extensions to $\Lambda$ CDM, as in Table V. Left: the $\Omega_{m}-\Omega_{\Lambda}$ constraints for a cosmological model under the assumption of a $w=-1$ cosmological constant with free curvature (o $\Lambda \mathrm{CDM})$. Right: the $w-\Omega_{m}$ constraints for a flat cosmological model where the equation of state is allowed as a constant, free parameter. In both cases, the gray contours represent the $68 \%$ and $95 \%$ confidence intervals using only the Planck temperature and polarization data, while the blue contours show the results including RSD data. The combination of RSD, DES WL, Planck lensing, and $\mathrm{CMB}$ is shown in red.

TABLE VI. Marginalized values and 68\% confidence limits on curvature, dark energy parameters, and the amplitude of density fluctuations using only growth and CMB temperature and polarization measurements.

\begin{tabular}{|c|c|c|c|c|c|c|}
\hline & & $\Omega_{m}$ & $\Omega_{\mathrm{DE}}$ & $\sigma_{8}$ & $\Omega_{k}$ & $w$ \\
\hline \multirow[t]{4}{*}{$\mathrm{o} \Lambda \mathrm{CDM}$} & CMB T\&P & $0.483_{-0.069}^{+0.055}$ & $0.561_{-0.041}^{+0.050}$ & $0.774_{-0.014}^{+0.016}$ & $-0.044_{-0.014}^{+0.019}$ & $\cdots$ \\
\hline & $\mathrm{CMB} \mathrm{T} \& \mathrm{P}+\mathrm{RSD}$ & $0.456_{-0.066}^{+0.051}$ & $0.580_{-0.038}^{+0.048}$ & $0.780_{-0.013}^{+0.015}$ & $-0.037_{-0.013}^{+0.018}$ & $\cdots$ \\
\hline & CMB T \& P + WL & $0.310 \pm 0.017$ & $0.690 \pm 0.013$ & $0.806 \pm 0.010$ & $-0.0004 \pm 0.0048$ & $\cdots$ \\
\hline & CMB T \& $\mathrm{P}(+$ lens $)+\mathrm{RSD}+\mathrm{WL}$ & $0.313 \pm 0.014$ & $0.688 \pm 0.011$ & $0.8069 \pm 0.0096$ & $-0.0010_{-0.0039}^{+0.0044}$ & $\cdots$ \\
\hline \multirow[t]{4}{*}{$w \mathrm{CDM}$} & CMB T\&P & $0.199_{-0.057}^{+0.022}$ & $0.801_{-0.022}^{+0.057}$ & $0.970_{-0.045}^{+0.096}$ & $\cdots$ & $-1.58_{-0.35}^{+0.16 a}$ \\
\hline & $\mathrm{CMB} \mathrm{T} \& \mathrm{P}+\mathrm{RSD}$ & $0.295_{-0.034}^{+0.028}$ & $0.705_{-0.028}^{+0.022}$ & $0.834 \pm 0.030$ & $\cdots$ & $-1.08 \pm 0.11$ \\
\hline & $\mathrm{CMB} \mathrm{T} \& \mathrm{P}+\mathrm{WL}$ & $0.188_{-0.046}^{+0.012}$ & $0.812_{-0.012}^{+0.046}$ & $0.977_{-0.037}^{+0.083}$ & $\cdots$ & $-1.61_{-0.30}^{+0.13}$ \\
\hline & CMB T \& $\mathrm{P}(+$ lens $)+\mathrm{RSD}+\mathrm{WL}$ & $0.275_{-0.028}^{+0.024}$ & $0.725_{-0.024}^{+0.012}$ & $0.846 \pm 0.028$ & $\cdots$ & $-1.14 \pm 0.10$ \\
\hline
\end{tabular}

\footnotetext{
${ }^{\mathrm{a}}$ The lower bound on $w$ is affected by the $H_{0}$ prior.
} 
in the low-redshift regime. The WL measurements, when combined with the CMB data, substantially shift the constraints on curvature to be consistent with flatness $\left(\Omega_{k}=-0.0004 \pm 0.0048\right)$, with a factor of 3.4 reduction in uncertainty. The constraining power of CMB lensing lies in between the RSD and low-redshift WL; combining the Planck lensing with the temperature and polarization data leads to a best-fit model consistent with the $\Lambda$ CDM model $\left(\Omega_{k}=-0.011 \pm 0.006\right.$; [46]).

\section{Expansion history and dark energy}

We next explore the constraints on a flat $w \mathrm{CDM}$ model, where the equation of state $w$ for dark energy is constant but allowed to vary. The Planck temperature and polarization data prefer a value of $w$ much more negative than -1 , and adding CMB lensing causes virtually no change [46]. As shown in the right panel of Fig. 8, the combination of growth measurements with CMB data provides constraints on $w$ that enclose the cosmological constant model within the $95 \%$ contours. Contrary to the case of o $\Lambda \mathrm{CDM}$, it is the RSD data that have the largest impact in shifting the CMB contours. As shown in Table V, combining WL measurements with $\mathrm{CMB}$ does not significantly improve the precision on $w$, but RSD measurements are able to improve the precision by more than a factor of 2 .

The constraining power of RSD on $w$ can be understood from Fig. 7. A more negative value of $w$ causes increasingly slower structure growth toward lower redshifts. The $f \sigma_{8}$ measurements with BOSS and eBOSS galaxies sample the growth rate in the redshift range $0.2<z<1$. good constraints on the shape of $f \sigma_{8}(z)$ around its peak and thus constraints on $w$. The CMB + RSD data are therefore able to rule out (at 4.5 standard deviations) the formal central value of $w=-1.585$ preferred by CMB alone. The combination of all the growth measurements with $\mathrm{CMB}$ prefers a model consistent with $w=-1$, at the level of $1.4 \sigma$, when using the one-dimensional, marginalized likelihoods.

We see that $\mathrm{CMB}$ and growth measurements provide factor of 2.5-4 improvements on the precision in extended $\Lambda \mathrm{CDM}$ models when compared to CMB temperature and polarization data alone. The growth data have the net effect of pulling the CMB data closer to a $\Lambda \mathrm{CDM}$ model.

\section{B. RSD constraints on the amplitude of matter fluctuations and tests of gravity}

Within the $\Lambda$ CDM model, RSD and lensing provide a means to integrate the rate of structure growth to redshift zero and estimate the current amplitude of matter fluctuations, $\sigma_{8}$. This estimate of $\sigma_{8}$ can be compared to the predictions when extrapolating the amplitude of the measured CMB power spectrum, thus serving as a $\Lambda \mathrm{CDM}$ consistency test similar to that of the $H_{0}$ inverse distance ladder tests in Sec. IV B. Structure growth can also be used to test the basic assumptions of the $\Lambda \mathrm{CDM}$ model through modifications to GR. In this case, the redshift evolution of matter density fluctuations and the interaction of matter and photons with the resultant gravitational potential can be directly compared to predictions of GR.

Here, we use SDSS RSD measurements, DES WL measurements, and Planck CMB lensing results to assess the amplitude of local matter fluctuations and perform a consistency test for GR.

\section{1. $R S D$ constraints on the amplitude of matter fluctuations}

First, we explore the constraints on $\Omega_{m}-\sigma_{8}$ from growth measurements assuming a $\Lambda \mathrm{CDM}$ cosmology. As shown in the left panel of Fig. 9, the constraints from each of the growth measurements (RSD, DES WL, and CMB lensing) are consistent with the predictions of models informed only by the $\mathrm{CMB}$, albeit with much larger contours. Note that we have applied conservative priors on $n_{s}$ and $\omega_{b}$ for all contours (see Appendix B).

The degeneracy from growth measurements follows the direction of lower $\Omega_{m}$ (thus slower structure growth) and higher-fluctuation amplitude $\sigma_{8}$. The differences in the degeneracy directions with RSD, WL, and CMB lensing measurements result from their different dependences on cosmology and different redshift sensitivities. Given the differences, we do not seek to present constraints on optimal combinations of the two parameters. Among the three growth measurements, RSD appears to have the largest contour area but provide the tightest constraints on $\sigma_{8}$, with $\sigma_{8}=0.836_{-0.062}^{+0.053}$. The WL measurements lead to overall better constraints in the $\Omega_{m}-\sigma_{8}$ plane, while the marginalized constraints on $\Omega_{m}$ are comparable to RSD, and those on $\sigma_{8}\left(\sigma_{8}=0.857_{-0.136}^{+0.163}\right)$ are not as tight as RSD. CMB lensing results in constraints in a direction similar to that of RSD, but with a stronger $\Omega_{m}-\sigma_{8}$ degeneracy and thus narrower contours than RSD.

The combination of RSD, WL, and CMB lensing is shown in the light purple contours in the left panel of Fig. 9. The resulting constraints are greatly improved, offering $\sigma_{8}=0.842_{+0.043}^{+0.034}$ and $\Omega_{m}=0.261_{-0.027}^{+0.036}$. In addition, the $68 \%$ confidence intervals overlap the $68 \%$ confidence intervals from the prediction based on CMB temperature and polarization data, indicating general consistency.

\section{2. $R S D$ constraints on modified gravity}

The difference between the speed of gravity and the speed of light has been shown to be negligible [198], as predicted by GR. $f \sigma_{8}(z)$ measurements from RSD can be used to further test theories of gravity in the context of structure formation.

Here, we consider a phenomenological parametrization of gravity, as described in Sec. II B, allowing for the two metric potentials $\Psi$ and $\Phi$ to deviate from their GR prediction, independent of the speed of gravitational waves. 

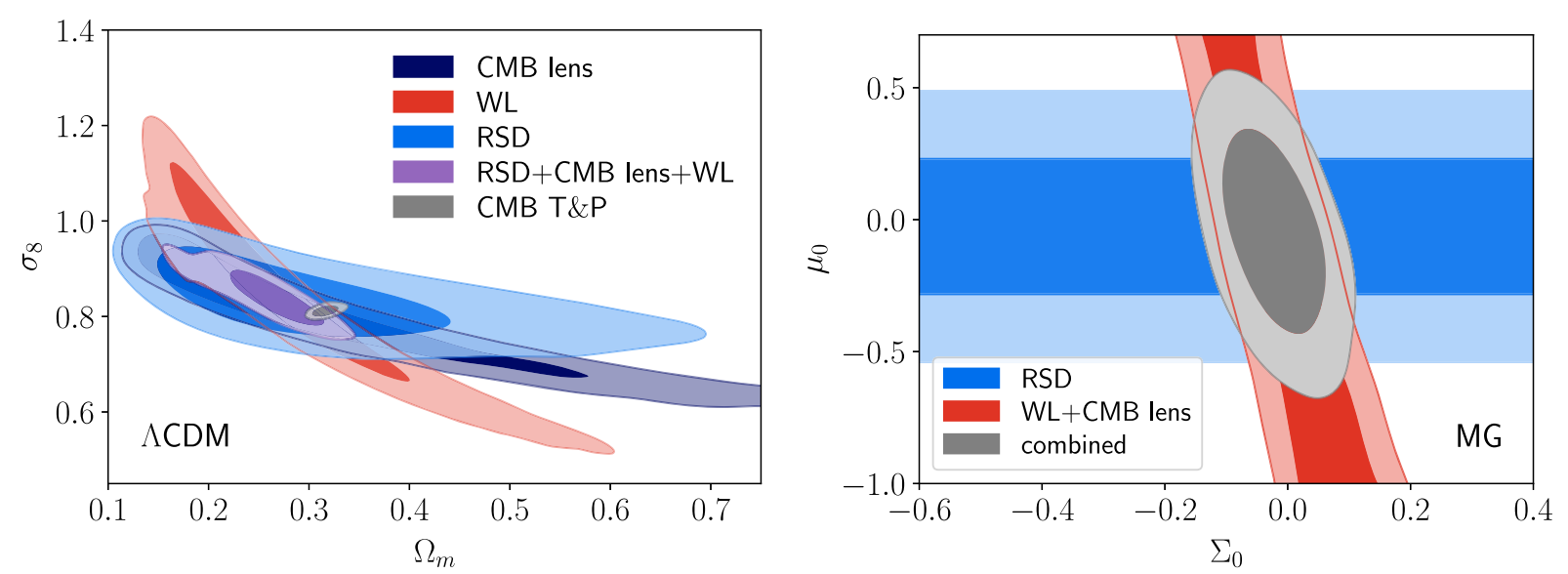

FIG. 9. Left: the $\Omega_{m}-\sigma_{8}$ constraints for a $\Lambda$ CDM cosmology. The blue contours represent the $68 \%$ and $95 \%$ constraints when using only the RSD sample. The red contours represent the same when using only the DES WL results. The dark blue contours represent the constraints from Planck lensing, while the light purple represent the combination of RSD, DES WL, and Planck lensing. The gray contours represent the predictions from the Planck temperature and polarization data under an assumption of a $\Lambda$ CDM cosmological model. Note that the contours for low values of $\Omega_{m}$ are affected by the $H_{0}$ prior. Right: the $\Sigma_{0}-\mu_{0}$ constraints for a cosmology with a fixed cosmological constant and perturbations to GR as described in Eqs. (11) and (12). The blue contours represent the 68\% and 95\% constraints when using only the RSD measurements. The red contours represent the same when using the DES WL and Planck lensing data. The gray contours represent the constraints when combining RSD, DES WL, and Planck lensing measurements.

The parameter $\mu_{0}$ characterizes the deviation of $\Psi$, which determines the response of matter to the gravitational potential and thus can be probed by RSD. The parameter $\Sigma_{0}$ characterizes the deviation of $\Psi+\Phi$, which determines the propagation of light and thus can be probed by lensing. Therefore, the combination of varying $\mu_{0}$ and $\Sigma_{0}$ provides us with a null test of gravity along the degeneracy direction of our most potent probes of modified gravity, RSD and lensing (WL and CMB lensing).

Assuming the fiducial cosmological model to be $\Lambda \mathrm{CDM}$ with the background parameters fixed to the baseline values (see Appendix B), we compute the constraints on $\mu_{0}$ and $\Sigma_{0}$ (right panel of Fig. 9). We use MGCAMB [199] [200] to allow for these phenomenological tests of general relativity. As expected, $\mathrm{WL}+\mathrm{CMB}$ lensing mainly constrain $\Sigma_{0}$, while RSD is only sensitive to $\mu_{0}$. A combination of both probes is necessary to break degeneracies between the two parameters. With the combined RSD and lensing, we find $\mu_{0}=-0.05 \pm 0.24$ and $\Sigma_{0}=-0.024 \pm 0.054$, consistent with the GR prediction of $\mu_{0}=\Sigma_{0}=0$.

\section{GLOBAL FITS}

After examining the impacts of expansion history and growth measurements alone, we now proceed to combine the Planck (including lensing), Pantheon SNe Ia, SDSS, and DES data to determine the best fitting cosmological model. The SDSS data consist of the combined BAO and RSD measurements found in the bottom section of Table III, while the DES data consist of the cosmic shear, galaxy clustering, and galaxy-galaxy lensing data (i.e., $3 \times 2 \mathrm{pt}$ ). We refer to the results from the previous sections where there is guidance on which datasets are providing the critical information.

We start by establishing the parameters for the simplest cosmology, that of a $\Lambda \mathrm{CDM}$ universe with a fixed neutrino mass. We examine the distribution of BAO and RSD measurements about this model to assess potential tension with any of the individual measurements.

We then expand the cosmological model to include free parameters for curvature, the dark energy equation of state, and the neutrino mass. In all cases, the best fitting values of $H_{0}$ are determined at a precision of better than $0.8 \mathrm{~km} \mathrm{~s}^{-1} \mathrm{Mpc}^{-1}$. We then show that the addition of these free parameters does not lead to significant changes in any of the $\Lambda \mathrm{CDM}$ parameters and that the results remain consistent with a flat $\Lambda \mathrm{CDM}$ universe. Finally, we provide a physical interpretation of the cosmology constraints on summed neutrino mass in the context of neutrino oscillation experiments.

\section{A. $\Lambda$ CDM model}

We start by finding the $\Lambda$ CDM model that best describes the full suite of data. As shown in the first row of Table VII, the dark energy density is constrained at the level of $0.7 \%$. This precision is improved by a factor of 1.28 over the value found in Table IV for a combination of CMB, BAO, and SN and a factor of 1.79 over the $\mathrm{CMB}$ data alone, indicating that the dark energy density constraints are dominated by the expansion history measurements.

The precision of $\Lambda \mathrm{CDM}$ parameter constraints allows us to evaluate the distribution of SDSS measurements about the model. For the purpose here, we use Gaussian 
TABLE VII. Marginalized values and 68\% confidence limits for models using Planck, Pantheon SNe, SDSS BAO + RSD, and DES $3 \times 2 \mathrm{pt}$ data.

\begin{tabular}{|c|c|c|c|c|c|c|c|}
\hline & $\Omega_{\Lambda}$ & $H_{0}$ & $\sigma_{8}$ & $\Omega_{\mathrm{K}}$ & $w_{0}$ & $w_{a}$ & $\Sigma m_{\nu}[\mathrm{eV}]^{\mathrm{a}}$ \\
\hline$\Lambda \mathrm{CDM}$ & $0.6960 \pm 0.0047$ & $68.19 \pm 0.37$ & $0.8072 \pm 0.0056$ & $\ldots$ & $\cdots$ & $\cdots$ & $\cdots$ \\
\hline $\mathrm{o} \Lambda \mathrm{CDM}$ & $0.6959 \pm 0.0046$ & $68.24 \pm 0.54$ & $0.8076 \pm 0.0065$ & $0.0002 \pm 0.0017$ & $\ldots$ & $\cdots$ & $\cdots$ \\
\hline$w \mathrm{CDM}$ & $0.6996 \pm 0.0066$ & $68.68 \pm 0.73$ & $0.8130 \pm 0.0093$ & $\ldots$ & $-1.021 \pm 0.027$ & .. & $\ldots$ \\
\hline owCDM & $0.6998 \pm 0.0071$ & $68.62 \pm 0.75$ & $0.8126 \pm 0.0093$ & $-0.0003 \pm 0.0019$ & $-1.023 \pm 0.031$ & $\ldots$ & $\cdots$ \\
\hline$w_{0} w_{a} \mathrm{CDM}$ & $0.6973 \pm 0.0069$ & $68.49 \pm 0.75$ & $0.8141 \pm 0.0094$ & $\ldots$ & $-0.938 \pm 0.073$ & $-0.32_{-0.24}^{+0.28}$ & $\ldots$ \\
\hline $\mathrm{o} w_{0} w_{a} \mathrm{CDM}$ & $0.6984 \pm 0.0070$ & $68.18 \pm 0.79$ & $0.8136 \pm 0.0092$ & $-0.0022 \pm 0.0022$ & $-0.909 \pm 0.081$ & $-0.49_{-0.30}^{+0.35}$ & $\cdots$ \\
\hline$\nu \Lambda \mathrm{CDM}$ & $0.6974_{-0.0049}^{+0.0054}$ & $68.33_{-0.39}^{+0.45}$ & $0.8113_{-0.0068}^{+0.0093}$ & $\ldots$ & $\ldots$ & & $<0.115$ \\
\hline$\nu w \mathrm{CDM}$ & $0.6993 \pm 0.0066$ & $68.63 \pm 0.72$ & $0.813_{-0.0099}^{+0.011}$ & $\cdots$ & $-1.018_{-0.028}^{+0.033}$ & $\cdots$ & $<0.162$ \\
\hline
\end{tabular}

${ }^{\mathrm{a}}$ The reported $\sum m_{\nu}$ values correspond to the $95 \%$ upper limits.

approximations to the measurements for the evaluation. In comparison with the best-fit model, the 14 BAOonly measurements $\left(D_{V} / r_{d}, D_{M} / r_{d}\right.$, and $D_{H} / r_{d}$; see Table III) give a value of $\chi^{2}=11.0$, with covariance among measurements taken into account. Similarly, the six RSD measurements (Table III) give $\chi^{2}=6.6$. Finally, we consider the full set by combining the 17 $\mathrm{BAO}+\mathrm{RSD}$ measurements with the four BAO measurements from $\operatorname{Ly} \alpha$-Ly $\alpha$ and $\operatorname{Ly} \alpha$-Quasar correlations and obtain $\chi^{2}=23.7$. Based on the $\chi^{2}$ distribution with 14,6 , and 21 degrees of freedom, respectively, all sets of measurements are fully consistent with the preferred model.

To evaluate whether there is any statistically significant outlier in the measurements, we compute the residual between each SDSS measurement and the value predicted by the preferred model. In this pull distribution, the residuals are normalized by the measurement uncertainty, so one would expect a Gaussian distribution with unity width if the measurements were distributed according to the measurement uncertainties. We account for the correlations among measurements by diagonalizing each covariance matrix to produce statistically independent pull values. The resulting distribution of the pull values is shown in the left panel of Fig. 10. For the BAO-only measurements, the pull with the largest deviation, -1.75 , comes from the $z \sim 0.7$ eBOSS LRG sample. For the RSD measurements, the largest deviation, 1.91, is from the $z \sim 1.48$ eBOSS Quasar sample. For the full set (labeled as "BAO + RSD"), the largest deviation is again from the eBOSS Quasar sample. After accounting for the covariance between BAO and RSD measurements, the measurement differs from the $\Lambda \mathrm{CDM}$ prediction by 3.0 standard deviations. Based on the Kolmogorov-Smirnov (KS) test with the cumulative distribution (right panel of Fig. 10), the pull distributions for $\mathrm{BAO}, \mathrm{RSD}$, and $\mathrm{BAO}+\mathrm{RSD}$ measurements are found to be consistent with the normal distribution, revealing no unexpected feature in the measurements for a universe best described by the $\Lambda \mathrm{CDM}$ model. Additional diagnostics using the Hubble parameter can serve as consistency check on dark energy constraints (e.g., Ref. [201]) as has been done to assess the BOSS BAO results [202]. Preliminary results also indicate that the eBOSS data are consistent with a $\Lambda$ CDM model.

Within the $\Lambda$ CDM model, the SDSS, DES, and Planck data offer tests of GR predictions on growth rates and modeldependent predictions for $H_{0}$. The left panel of Fig. 11 shows the constraints from these three programs on the amplitude of the (linear) power spectrum, while the right panel shows the constraints on $H_{0}$. In a $\Lambda \mathrm{CDM}$ cosmology, the model describing the DES $3 \times 2$ pt data has a strong degeneracy between $\sigma_{8}$ and $\Omega_{m}$ and a strong degeneracy between $H_{0}$ and $\Omega_{m}$. In both cases, the DES data are described by somewhat lower values of $\Omega_{m}$ than the model describing the CMB data. The mild tension that has been reported between lensing and $\mathrm{CMB}$ estimates of $\sigma_{8}$ can therefore be equally explained by the preference in WL estimates for a $\Omega_{m}$ value lower than 0.3. The $\sigma_{8}$ and $\Omega_{m}$ degeneracy is similar to that found in the KiDS weak lensing analysis [203] and in the HSC weak lensing analysis [43], both of which prefer lower values of $\sigma_{8}$ if evaluated with a CMB prior on $\Omega_{m}$.

Other studies have recently used the same BOSS galaxy data we use in order to obtain results on $\sigma_{8}$ and $\Omega_{m}$ when
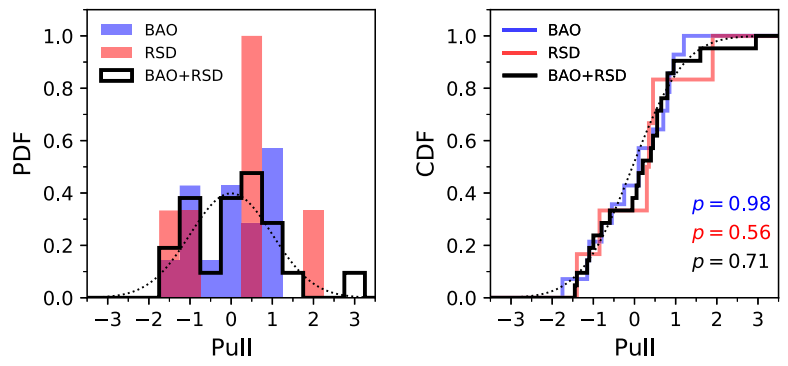

FIG. 10. Distribution of residuals of the SDSS BAO (blue), RSD (red), and BAO + RSD (black) measurements with respect to the best-fit $\Lambda \mathrm{CDM}$ model. In all cases, the residuals are represented in the form of pulls, i.e., normalized by the measurement uncertainty. The left panel shows the probability distribution, and the right panel shows the cumulative distribution. The $p$ values are from KS tests in comparison with the normal distribution (dotted curves). 

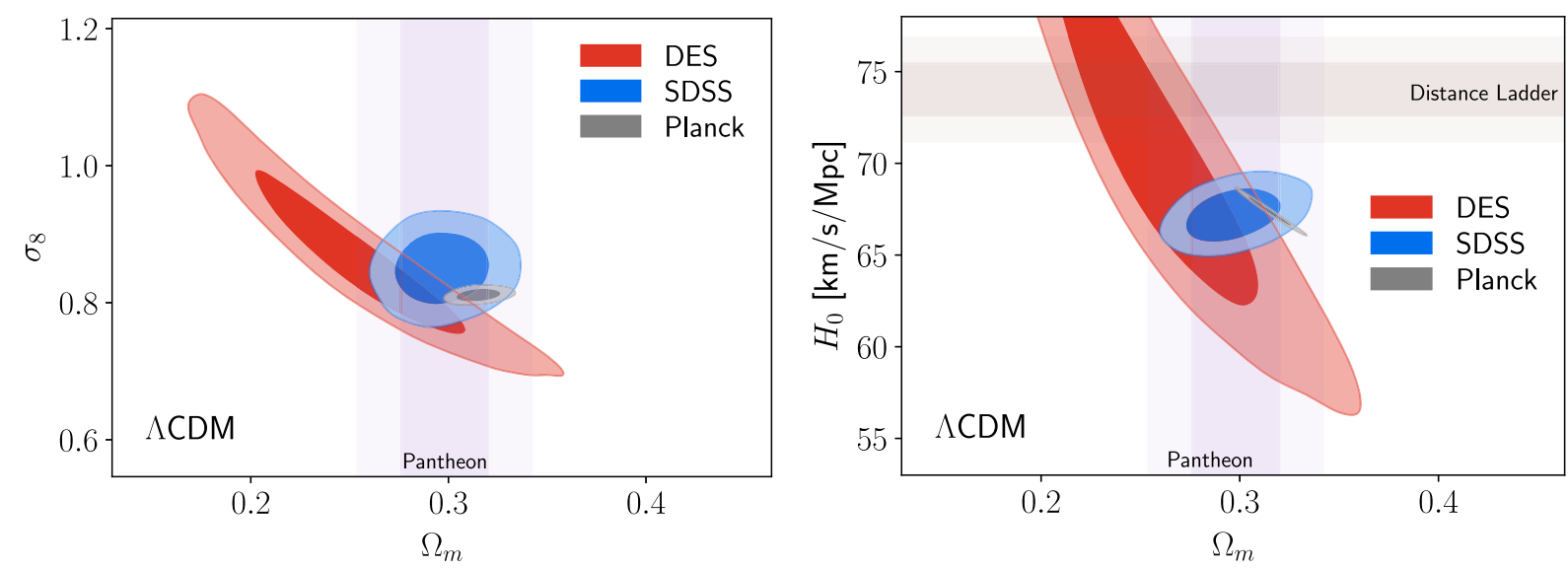

FIG. 11. Left: the $\Omega_{m}-\sigma_{8}$ constraints for a $\Lambda$ CDM model. A BBN-inspired prior on $\omega_{b}$ and a prior of $n_{s}=0.96 \pm 0.02$ was assumed for the SDSS and DES contours. Right: $H_{0}$ vs $\Omega_{m}$ under a $\Lambda$ CDM model. In both panels, the $68 \%$ and $95 \%$ confidence intervals for the $\mathrm{BAO}+\mathrm{RSD}$ data are shown in blue, the DES $3 \times 2$ pt data are shown in red, and the Planck CMB and lensing data are shown in gray. The faint, vertical purple bands represent the Pantheon constraints of $\Omega_{m}=0.298 \pm 0.022$ [114]. In the right panel, the faint, brown horizontal bands represent the Cepheid/SNe Ia measurements from Riess et al. [45], $H_{0}=74.03 \pm 1.42 \mathrm{~km} \mathrm{~s}^{-1} \mathrm{Mpc}^{-1}$.

restricting to a $\Lambda$ CDM cosmology [204-206]. These studies have found lower central values of $\sigma_{8}$ than what we find in our measurements from SDSS clustering or what would be expected from Planck measurements. A recent example is that found in the KiDS weak lensing analysis [203]. In this study, BOSS galaxy clustering measurements over redshift intervals $0.2<z<0.5$ and $0.5<z<0.75$ were used to further constrain $\sigma_{8}$ and $\Omega_{m}$ and reduce the degeneracy between the two parameters. They find $S_{8}=0.766_{-0.014}^{+0.020}$, lower by $8.3 \pm 2.6 \%$ relative to Planck predictions. Their result using only the BOSS DR 12 clustering data leads to an estimate $\sigma_{8}=0.75_{-0.047}^{+0.045}$, about two standard deviations lower than the central value in result from all SDSS clustering $\left(\sigma_{8}=0.85 \pm 0.03\right)$.

In addition to the difference in methodologies, the SDSS dataset includes a larger number of tracers over a larger redshift range. We include results from six redshift regimes and include postreconstruction BAO measurements where possible. Even restricting to BOSS, the data we include is substantially different, as we use the results from the BOSS $0.4<z<0.6$ dataset, and we do not directly use the $0.5<$ $z<0.75$ BOSS galaxy clustering data. Instead, the BOSS $z>0.6$ data were combined with the eBOSS LRG sample [68]. The additional data generally favors $f \sigma_{8}$ values that are greater than those preferred by Planck (Fig. 7). Our SDSS result thus represents the most complete representation of structure growth constraints from galaxy clustering data and indicates no tension with Planck CMB measurements, albeit with an implied prior that the shape of the linear power spectrum matches that observed by the CMB.

\section{B. Constraints on dark energy and curvature}

As was demonstrated in Sec. IV, the main strength of the $\mathrm{BAO}$ and $\mathrm{SN}$ distance measurements is to constrain cosmological models with free curvature and varying dark energy equation of state, respectively. As was shown in Sec. V, the main strength of the growth measurements is in constraining possible deviations from GR. We now explore the complementarity of distance and growth measurements by testing the same single-parameter extensions to $\Lambda \mathrm{CDM}$ that were presented in Sec. IV, followed by models with increasing degrees of freedom. The results are found in Table VII.

\section{1. $\Omega_{\mathrm{DE}}, H_{0}$, and $\sigma_{8}$ parameters}

First, the central values of the three parameters, $\Omega_{\mathrm{DE}}$, $H_{0}$, and $\sigma_{8}$, are all consistent with the prediction from the best-fit $\Lambda$ CDM model (Table VII) at $68 \%$ confidence, regardless of the cosmological model that is assumed. The largest fractional deviation from the $\Lambda \mathrm{CDM}$ prediction is only $0.8 \%$, in the case of $\sigma_{8}$ in the $0 w_{0} w_{a} \mathrm{CDM}$ model. That measurement is fully consistent with the $\Lambda \mathrm{CDM}$ prediction of $\sigma_{8}=0.8120 \pm 0.0073$ from $\mathrm{CMB}$ data alone [46]. The robustness of $\sigma_{8}$ measurements to cosmological model provide further evidence that the growth of structure can be described using GR in a $\Lambda$ CDM parametrization.

In addition, the precision on the three parameters does not degrade significantly between the $\Lambda \mathrm{CDM}$ model and expanded models. When expanding to the $\mathrm{o} w_{0} w_{a} \mathrm{CDM}$ model, the precision on the $\Omega_{\mathrm{DE}}$ and $H_{0}$ parameters degrades by factors of 1.5 and 2.1, respectively. The largest degradation for $\sigma_{8}$ precision occurs with the $\nu w \mathrm{CDM}$ model, leading to a factor of 1.8 increase in the uncertainties. The tight constraints offered in all models are a result of the complementarity between observational probes.

As discussed in Sec. IV, interesting tensions appear between the estimates of the current Hubble expansion rate 
from local measurements and from extrapolations of the calibrated drag scale to $z=0$ using the CMB. Those estimates of a low $H_{0}$ extrapolated from early times are not changed with the addition of the growth data. For even the most flexible $0 w_{0} w_{a} \mathrm{CDM}$ cosmology, we find $H_{0}=68.18 \pm 0.79 \mathrm{~km} \mathrm{~s}^{-1} \mathrm{Mpc}^{-1}$, consistent with the findings in Sec. IV. The addition of the growth data leads to a $9 \%$ improvement in the precision on $H_{0}$ compared to the results using $\mathrm{CMB} \mathrm{T} \& \mathrm{P}+\mathrm{BAO}+\mathrm{SN}$.

None of the extended models improves the best-fit $\chi^{2}$ by more than 4 compared to the $\chi^{2}$ of the $\Lambda$ CDM model of $\chi^{2}=4366$, even with the introduction of additional degrees of freedom. We find a mild change in the $\chi^{2}$ for models with free dark energy equation of state, $w$, while models with curvature and varying time evolution of dark energy, $w_{a}$, only show a marginal change. Hence, we do not detect strong evidence for the need to extend the $\Lambda \mathrm{CDM}$ model.

\section{Curvature and dark energy}

When comparing the global results in an $\mathrm{o} \Lambda \mathrm{CDM}$ model to those from $\mathrm{CMB} \mathrm{T} \& \mathrm{P}+\mathrm{BAO}+\mathrm{SN}$ in Table IV, we find that the addition of the RSD, Planck lensing, and DES data only provides improvements of $6 \%$ on the precision of curvature constraints. The impact of growth measurements is larger in the $w \mathrm{CDM}$ model; the additional data provide improvements of about $22 \%$ on the precision for a constant equation of state. As discussed in Sec. V and in Fig. 7, the improvement is likely primarily from the RSD measurements.

When expanding to an evolving dark energy model with zero curvature, we find that the best-fit models are still consistent with $\Lambda \mathrm{CDM}$. The $w_{0} w_{a} \mathrm{CDM}$ model does not improve the fit relative to $\Lambda \mathrm{CDM}$, indicating that the additional free parameter is not providing critical new information. Overall, we find consistent constraints on $w_{0}$ with those in a $w \mathrm{CDM}$ model.

The complementarity of BAO/RSD and SNe Ia measurements is best demonstrated in expanded dark energy models that also allow for free curvature. We only find meaningful prior-independent constraints on the general $\mathrm{o} w_{0} w_{a} \mathrm{CDM}$ model for the combination of all datasets, as shown in Fig. 12. As shown in Table VII, the uncertainties on the two dark energy equation of state parameters in an o $w_{0} w_{a}$ CDM model are relatively unchanged when compared to results under the assumption of a spatially flat universe $\left(w_{0} w_{a} \mathrm{CDM}\right)$. The uncertainties on curvature are only increased by $29 \%$ when compared to the singleparameter $\mathrm{o} \Lambda \mathrm{CDM}$ extension.

In Fig. 12, it can be seen that the $w_{0}-\Omega_{k}$ confidence intervals from the Planck + SDSS data are orthogonal to the Planck $+\mathrm{SNe}$ contours. As was demonstrated in Sec. IVA, the BAO data best constrain the curvature, while the combination of $\mathrm{CMB}$ and $\mathrm{SNe}$ Ia best constrains the dark energy equation of state. As shown in the onedimensional likelihood of $\Omega_{k}$, the constraints on $\Omega_{k}$ are roughly three times better using Planck + SDSS than those using Planck $+\mathrm{SN}$. The Planck $+\mathrm{SN}$ data perform slightly better than do the Planck + SDSS data in the $w_{0}-w_{a}$ plane, but the net precision on both $w_{0}$ and $w_{a}$ increases by roughly a factor of 2 when combining all measurements. This statistical increase in constraining power is much larger than one would expect due to the contribution of the Planck + BAO data to provide tight constraints on curvature. Most importantly, the combination of all cosmological probes reveals again a preference for the $\Lambda$ CDM model. From the one-dimensional marginalized distributions, $w_{0}=-1$ is at 1.1 standard deviations, $w_{a}=0$ is at 1.3 standard deviations, and $\Omega_{k}=0$ is almost within the $68 \%$ confidence interval.

In a related CPL parametrization for dark energy, we can define a pivot scale factor $a_{p}$, or equivalently a pivot redshift $z_{p}$. Instead of evaluating the equation of state at $z=0$, as is done throughout this paper, we can represent the time-evolving equation of state as $w(a)=w_{p}+$ $w_{a}\left(a_{p}-a\right)$. Note that change of the pivot redshift does not change the model physically because the same linear relation can be described by the value and slope at any one point. However, by choosing the pivot scale appropriate to the redshifts covered by the data, constraints on $w_{p}$ and $w_{a}$ can be made to be nearly uncorrelated. In doing so, we find constraints in the $w_{0} w_{a} \mathrm{CDM}$ model $w_{p}=-1.020 \pm 0.029$ and $w_{a}=-0.32_{-0.24}^{+0.28}$ when using a pivot redshift $z_{p}=0.35$. The result demonstrates that we can constrain the dark energy equation of state to $3 \%$ precision at an earlier epoch in cosmic history. This precision is only degraded by a factor of 1.07 relative to the constraint on $w$ in an $w \mathrm{CDM}$ model, indicating that the overall effect of adding the additional parameter for a timevarying equation of state is minimal.

The results from joint fits can be used to compute a total Dark Energy Task Force Figure of Merit (FOM) [207] for various sample combinations in a model with time-varying equation of state. Computed as the inverse product of $w_{p}$ and $w_{a}$, the FOM associated with the full SDSS and Planck data is 37.5 in the $w_{0} w_{a} \mathrm{CDM}$ model. The FOM increases by a factor of 3.5 when adding the Pantheon SNe Ia and the DES $3 \times 2$ pt data. Demonstrating the complementarity of the $\mathrm{BAO}$ and $\mathrm{SNe}$ Ia data in constraining curvature and the dark energy equation of state, the Dark Energy FOM for all datasets only decreases from 132 in the $w_{0} w_{a} \mathrm{CDM}$ model to 94 in the $\mathrm{o} w_{0} w_{a} \mathrm{CDM}$ model.

\section{Neutrino mass}

The existence of neutrino oscillations has been confirmed by numerous terrestrial experiments [208-215]. These experiments measure the difference between the squares of neutrino mass eigenstates, leading to two sets of possible solutions for individual masses, which are referred to as the normal and inverted hierarchies. Both of these two 


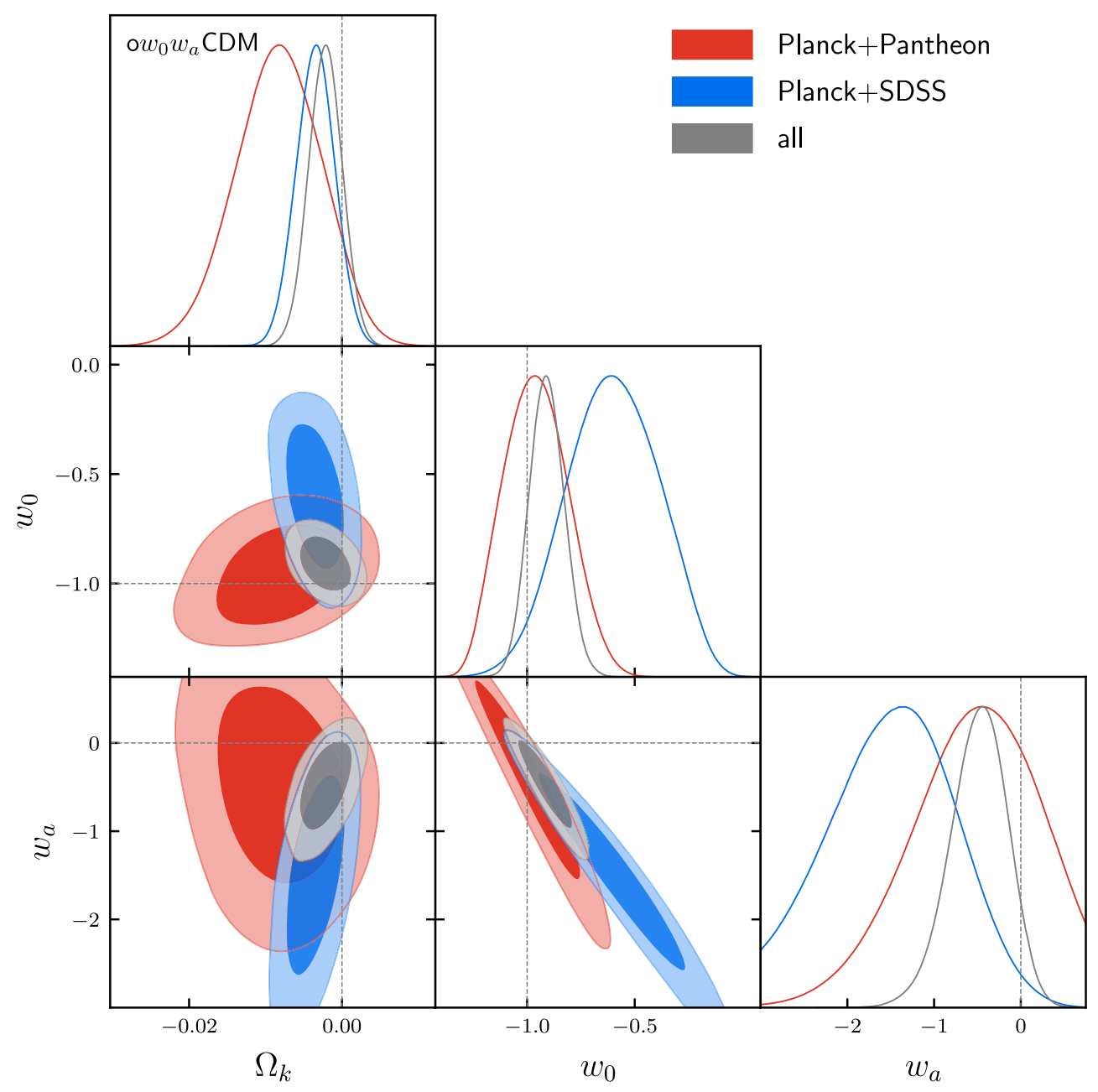

FIG. 12. Two-dimensional contours on $w_{0}, w_{a}$, and $\Omega_{k}$ under the assumption of an ow $w_{a}$ CDM cosmological model. The onedimensional constraints on each independent parameter are presented in the top panels. The red contours represent the $68 \%$ and $95 \%$ constraints when using the full Planck data (T\&P and lensing) and the Pantheon SNe Ia measurements. The blue contours represent the constraints from Planck and SDSS BAO + RSD, while the gray contours represent the combination of all measurements presented in this work.

solutions lead to degenerate neutrino masses if $\sum m_{\nu} \gtrsim$ $0.15 \mathrm{eV}$ but lead to different predictions at lower masses. For the normal hierarchy, the minimum neutrino mass is given by two essentially massless neutrinos and one massive neutrino. For the inverted hierarchy, the minimum mass is composed of one massless and two degenerate neutrinos. The constraints for these two scenarios are

$$
\begin{aligned}
& \sum m_{\nu}>0.0588 \mathrm{eV} \text { normal hierarchy, } \\
& \sum m_{\nu}>0.0995 \mathrm{eV} \text { inverted hierarchy }
\end{aligned}
$$

\section{[216,217].}

Throughout this paper, we assume the neutrino masses to be at the minimum mass $\sum m_{\nu}=0.06 \mathrm{eV}$ with one massive and two massless neutrinos. When allowing a free parameter to describe the neutrino mass, we continue to assume two massless and one massive neutrinos, which is a good approximation for the masses of interest $[90,218]$.

Resolving the hierarchy problem remains a key goal of ground-based neutrino experiments (e.g., Refs. [219-221]). Likewise, the goal of constraining the absolute mass of neutrinos has motivated a series of terrestrial experiments. The tightest constraints of direct measurements arise from the Katrin experiment [222], resulting in a $90 \%$ upper limit on the effective electron neutrino mass of $m\left(\nu_{e}\right)<1.1 \mathrm{eV}$.

Therefore, it is timely to address the status of neutrino mass constraints before the advent of Stage IV dark energy experiments. We show our results for the $\nu \Lambda \mathrm{CDM}$ and $\nu w C D M$ cosmological models in Table VIII with several quantities. The $95 \%$ upper limits are derived from Markov chains, containing a prior $\sum m_{\nu}>0$. In requiring only a mass that is positive, the cosmology constraints assume 


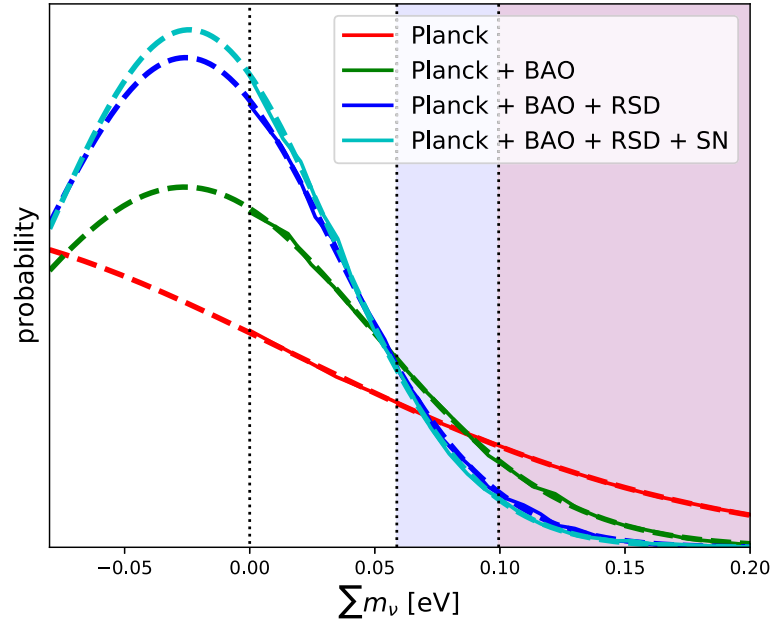

FIG. 13. Posterior for sum of neutrino masses for selected combinations of data with a $\nu \Lambda C D M$ cosmology. Dashed curves show the implied Gaussian fits. Shaded regions correspond to lower limits on normal and inverted hierarchies. Likelihood curves are normalized to have the same area under the curve for $\sum m_{\nu}>0$.

no prior information from the neutrino oscillation experiments and offer a fully independent measurement of neutrino mass. Four selected data combinations are plotted in Fig. 13.

It is useful to make a Gaussian approximation to better characterize the central values without influence from the prior and to provide a simple compression of the information for other analyses. These Gaussian fits are determined over the range $0<\sum m_{\nu}<0.15 \mathrm{eV}$ and are given in the last column of Table VIII. The upper 95\% limits from the fitted Gaussian posteriors are within $2 \%$ of the chains for $\Lambda \mathrm{CDM}$ and within $4 \%$ when $w$ is free to vary. While we see that the preferred neutrino mass implied by these fits is negative, a solution with $\sum m_{\nu}=0$ is usually within one standard deviation of the central value, and the minimal mass solution with the normal hierarchy is always within the $95 \%$ contours. Finally, the Gaussian variances on these fits are essentially second derivatives of the log-likelihood and are akin to Fisher matrix predictions. They can therefore be used to give insight into the constraining power of various probes that is free from the vagaries of the most likely position.

Because of the ability to break degeneracies with $\Omega_{m}$, the strongest improvement in neutrino mass precision over CMB-only constraints is caused by the addition of $\mathrm{BAO}$. This is due to reasons discussed at length in Ref. [192] and demonstrated in Sec. IVA. The RSD, which is the canonical neutrino probe for its ability to measure the suppression of growth due to freestreaming, improves the precision by another $26 \%$. Adding the RSD data is equivalent to an independent measurement with an error of about $0.1 \mathrm{eV}$ in $\Lambda \mathrm{CDM}$. The ability of BAO to improve upon $\mathrm{CMB}$ limits by breaking degeneracies with matter densities is essentially exhausted with the current generation of BAO experiments, as indicated in the right panel of Fig. 4. While currently not the dominant source of information on neutrinos, RSD should become the main probe with arrival of the new data in the next decade.

In Table VIII, we also show several integrated probabilities defined as

$$
\begin{gathered}
P_{\mathrm{norm}}=\int_{0.0588 \mathrm{eV}}^{\infty} p\left(m_{\nu}\right) d m_{\nu}, \\
P_{\mathrm{inv}}=\int_{0.0995 \mathrm{eV}}^{\infty} p\left(m_{\nu}\right) d m_{\nu}, \\
P_{\text {unphy }}=\int_{0}^{0.0588 \mathrm{eV}} p\left(m_{\nu}\right) d m_{\nu} .
\end{gathered}
$$

Note that these are not Bayesian evidences because we do not account for the prior volume. Nevertheless, the ratio of $P_{\text {inv }} / P_{\text {norm }}$ is the relative probability of the true mass lying in the range allowed by the inverted/normal hierarchy and is equivalent to an evidence ratio when the priors are very wide. The quantity $P_{\text {unphy }}$ is the probability of the summed neutrino mass lying in the unphysical region, with a mass lower than allowed by the normal hierarchy. We see that these probabilities are always inconclusive; there is no strong evidence from cosmology on a preference for a

TABLE VIII. Constraints on neutrino masses and relative probabilities of neutrino models with $\nu \Lambda C D M$ and $\nu w C D M$ cosmological models. The $95 \%$ upper limits are derived assuming a $\sum m_{\nu}>0$ prior.

\begin{tabular}{lcccc}
\hline \hline Data & $95 \%$ upper limit $(\mathrm{eV})$ & $P_{\text {inv }} / P_{\text {norm }}$ & $P_{\text {unphy }}$ & Gaussian fit (eV) \\
\hline Planck & 0.252 & 0.64 & 0.43 & \\
Planck + BAO & 0.126 & 0.36 & 0.64 & $-0.026 \pm 0.074$ \\
Planck + BAO + RSD & 0.101 & 0.24 & 0.76 & $-0.026 \pm 0.060$ \\
Planck + SN & 0.170 & 0.49 & 0.56 & $-0.076 \pm 0.106$ \\
Planck + BAO + RSD + SN & 0.097 & 0.22 & 0.78 & $-0.024 \pm 0.057$ \\
Planck + BAO + RSD + SN + DES & 0.115 & 0.27 & 0.71 & $-0.014 \pm 0.061$ \\
Planck + BAO + RSD + SN $(\nu$ CDM $)$ & 0.138 & 0.40 & 0.61 & $-0.033 \pm 0.082$ \\
Planck + BAO + RSD + SN + DES $(\nu w C D M)$ & 0.162 & 0.48 & 0.56 & $-0.048 \pm 0.097$ \\
\hline \hline
\end{tabular}


normal hierarchy, an inverted hierarchy, or a model where the neutrino mass is anomalously low (with or without allowing extrapolation into the negative $\sum m_{\nu}$ ). We also note that a $95 \%$ upper limit of less than $0.0995 \mathrm{eV}$ would not constitute a $2 \sigma$ detection of normal hierarchy because much of that posterior volume belongs to the unphysically low neutrino mass.

Evaluating the $95 \%$ upper limits, the strongest constraint excluding lensing data is $\sum m_{\nu}<0.097 \mathrm{eV}$, which degrades to $\sum m_{\nu}<0.115 \mathrm{eV}$ upon addition of lensing data. This reflects the shift toward a relatively low amplitude of $\sigma_{8}$ in the lensing data with the larger values of $\Omega_{m}$ preferred by the other probes.

Finally, we see that allowing the dark energy equation of state parameter $(w)$ to be free degrades the neutrino mass constraint by a factor of 1.4 to 1.6 . This effect is due to a known degeneracy direction in the neutrino mass [223]. Nevertheless, the effect is not as dramatic as it used to be, and with further data, it will become negligible.

\section{CONCLUSION}

The eight distinct samples of SDSS BAO measurements fill a unique niche in their ability to independently characterize dark energy and curvature in one-parameter extensions to $\Lambda \mathrm{CDM}$. When combined with Planck temperature and polarization data, the BAO measurements allow an order of magnitude improvement on curvature constraints when compared to Planck data alone. The BAO data provide strong evidence for a nearly flat geometry and allow constraints on curvature that are now roughly 1 order of magnitude within the detectable limit of $\sigma\left(\Omega_{k}\right) \sim$ 0.0001 [86]. The SDSS BAO measurements demonstrate that the observed cosmic acceleration is best described by a dark energy equation of state that is consistent with a cosmological constant to better than $6 \%$ precision when combined with the Planck temperature and polarization data. Finally, the SDSS BAO measurements allow robust estimates of the current expansion rate, demonstrating $H_{0}<70 \mathrm{~km} \mathrm{~s}^{-1} \mathrm{Mpc}^{-1}$ at $95 \%$ confidence under standard assumptions of prerecombination physics, regardless of cosmological model. These $H_{0}$ results remain consistent, even without the Planck CMB data, as long as the $\Lambda \mathrm{CDM}$ model is assumed.

Beyond the distance-redshift relation, we have also demonstrated the complementary role of the six independent SDSS RSD measurements to DES and Planck lensing measurements. The SDSS RSD measurements tighten Planck temperature and polarization constraints on the dark energy equation of state by more than a factor of 2 ; the DES WL measurements tighten constraints on curvature by more than a factor of 3. Independent of any BAO or SNe Ia information on the expansion history, the CMB, RSD, and WL measurements present a history of structure growth that is best described by a standard $\Lambda \mathrm{CDM}$ cosmology and a GR model for gravity.
The tightest constraints on the cosmological model are found when combining current measurements of the expansion history, CMB, and growth of structure. This combination reveals a dark energy density measured to $0.7 \%$ precision under an assumed $\Lambda \mathrm{CDM}$ model. We find approximately $1 \%$ precision estimates on $\Omega_{\mathrm{DE}}, H_{0}$, and $\sigma_{8}$ with central values that barely change under any extension explored in Sec. VI. The best-fitting parameters in extended models remain consistent with a $\Lambda \mathrm{CDM}$ cosmology; the most flexible o $w_{0} w_{a} \mathrm{CDM}$ model indicates constraints $\Omega_{k}=-0.0022 \pm 0.0022, w_{0}=-0.909 \pm 0.081$, and $w_{a}=-0.49_{-0.30}^{+0.35}$. The Dark Energy FOM for the full combination, in a model that allows for curvature, is 94 , about $38 \%$ lower than what was predicted 14 years ago by the Dark Energy Task Force [207]. However, the assumptions of the Dark Energy Task Force included the final DES cosmology results, whereas we only included the results of the first year WL and clustering studies. If the final DES studies of SNe Ia, galaxy clusters, and WL can provide an additional 60\% increase in the FOM, then the Dark Energy Task Force predictions will be proven accurate. The combination of measurements also provides an independent constraint on the summed neutrino mass, leading to $\sum m_{\nu}<0.115 \mathrm{eV}$ at $95 \%$ confidence $(\nu \Lambda \mathrm{CDM})$, with a slight preference for a normal hierarchy of mass eigenstates over an inverted hierarchy. The dominant factors in this neutrino mass measurement are the constraints from $\mathrm{CMB}$ and $\mathrm{BAO}$, thus making the result robust against challenges in modeling the full shape of the power spectrum in clustering and lensing measurements.

At the high precision found here, cosmic acceleration remains most consistent with predictions from a cosmological constant. A deviation from consistency with a pure cosmological constant perhaps would have pointed toward specific dark energy and modified gravity models. However, since many of these models have parameter choices that make them indistinguishable from $\Lambda \mathrm{CDM}$, those models all can be made consistent with our observations. Nevertheless, the observed consistency with flat $\Lambda \mathrm{CDM}$ at the higher precision of this work points increasingly toward a pure cosmological constant solution, for example, as would be produced by a vacuum energy fine tuned to have a small value. This fine-tuning represents a theoretical difficulty without any agreed-upon resolution, and one that may not be resolvable through fundamental physics considerations alone [224,225]. This difficulty has been substantially sharpened by the observations presented here.

\section{A. Decade of dark energy}

The profound insight offered into the cosmological model is only possible after several generations of experimental effort. Experiments designed to study the nature of dark energy have steadily improved in technique, redshift coverage, and sample size. In particular, the Planck CMB experiment offered a significant boost in spatial coverage 

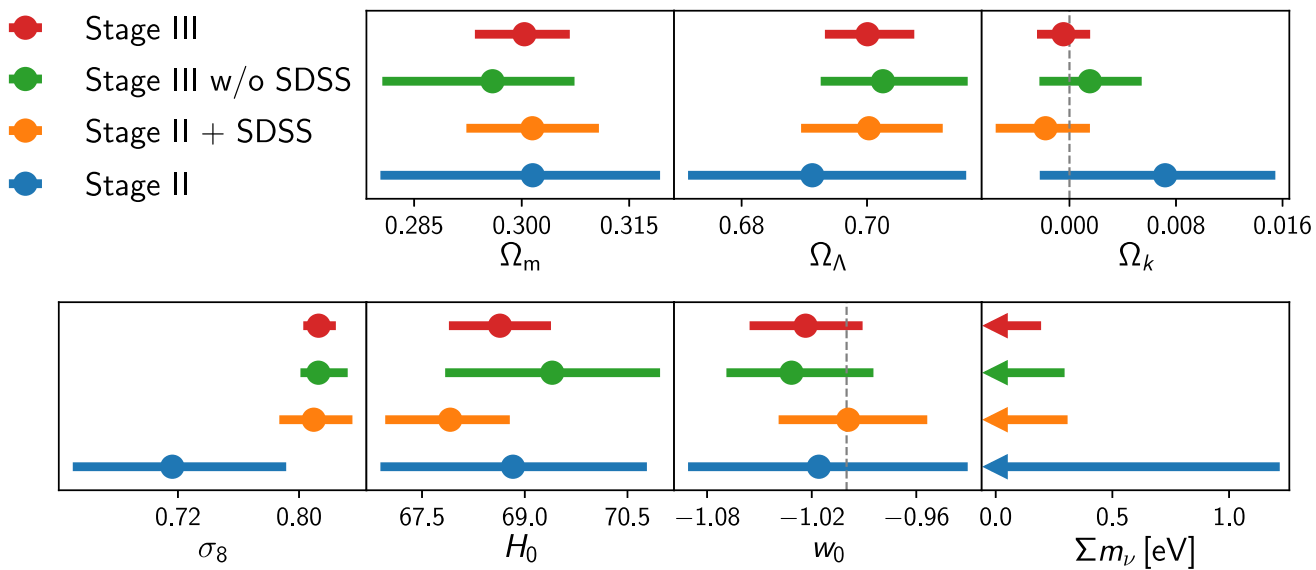

FIG. 14. Central values and $68 \%$ contours for each of the parameters describing expansion history and growth of structure in a $\nu$ ow CDM model. Results are shown for each dataset combination presented in the text, where Stage-II corresponds to a combination of the WMAP, JLA, and SDSS DR7 data and Stage-III corresponds to a combination of the SDSS BAO + RSD, Planck, Pantheon SN Ia, and DES $3 \times 2$ pt data.

and precision over WMAP, while the BOSS and eBOSS programs offered vast improvements in redshift range and statistical precision over the preceding spectroscopic surveys.

For a baseline to assess the impact of the current generation of dark energy experiments, we first characterize the dark energy constraints with the analogous programs that were concluding as BOSS was achieving first light. Representing the approximate period 2000-2010, we choose the final WMAP sample [16,54], the JLA sample of $\mathrm{SNe}$ Ia [113], and the $2.7 \%$ precision measurement of isotropic BAO at $z=0.275$ [226] from SDSS DR7 [127] and the 2-degree Field Galaxy Redshift Survey [11]. Following the convention presented in the report from the Dark Energy Task Force [207], we refer to this dataset as "Stage-II." Although some of these results were released as BOSS was nearing its conclusion, the data are representative of the previous generation of dark energy study.

The most recent cosmology results are reflected in the Planck temperature, polarization, and lensing data; the Pantheon SNe Ia sample; the SDSS BAO + RSD measurements; and the DES $3 \times 2$ pt samples. This dataset, referred to as "Stage-III," provides the main constraints presented in this paper and in Table VII.

Finally, we isolate the improvements over the Stage-II constraints from the SDSS BAO + RSD program. We do so by replacing the SDSS DR7 BAO measurements with the SDSS BAO + RSD measurements, while keeping the WMAP CMB and JLA SNe Ia samples intact. This combination is then referred to as "Stage-II+SDSS." In the same vein, we isolate the improvements over the Stage-II constraints from recent programs other than SDSS. Denoted "Stage-III w/o SDSS," the constraints are derived from the Stage-II programs; Planck temperature, polarization, and lensing data; the Pantheon SNe Ia sample; and the DES $3 \times 2$ pt samples.
Although the $\mathrm{o} w_{0} w_{a} \mathrm{CDM}$ model is the most flexible of all models explored in this work, with regard to dark energy parametrization, only the full Stage-III dataset is able to converge without strong priors that exclude unphysical values of $w_{a}$ (e.g., see Fig. 12). On the other hand, the three one-parameter extensions presented in Sec. IV demonstrate the complementarity between the probes in constraining a constant dark energy equation of state, curvature, and the neutrino mass. We therefore quantify advances of the last decade by computing cosmological constraints in a $\nu \mathrm{owCDM}$ model. The marginalized $68 \%$ confidence intervals for each of the key cosmological parameters in this model, for each of the relevant Stage-II and Stage-III sample combinations, is presented in Fig. 14.

The general effect of the Stage-III measurements is to push the Stage-II results closer to a $\Lambda$ CDM model in both curvature and the dark energy equation of state. The StageIII results also significantly reduce the upper bounds on the neutrino mass without any indication for a central value that is larger than $0 \mathrm{eV}$. With the exception of $\sigma_{8}$, the central values of all parameters in the Stage-III results overlap the $68 \%$ confidence intervals of the Stage-II results. The precision on all parameters has increased by at least a factor of 2.5. The largest gains from Stage-II to Stage-III are found in the constraints on $\Omega_{k}, \sigma_{8}$, and $\sum m_{\nu}$, with improvements in precision by factors of 4.5, 7.0, and 7.1, respectively.

We compute the relative gain across the full volume of the $68 \%$ confidence intervals on $w, \Omega_{k}$, $\sum m_{\nu}, H_{0}$, and $\sigma_{8}$. We use a figure of merit related to the inverse of the determinant of the covariance matrix for these five parameters. We define our figure of merit as $\mathrm{FOM}=|\operatorname{Cov}(\mathbf{p}, \mathbf{p})|^{-1 /(2 N)}$, where $N=5$ is the number of free parameters (represented by $\mathbf{p}$ ). This form properly tracks the typical gain in the $68 \%$ confidence interval for each free parameter. We find $\mathrm{FOM}=11,24$, and 44 for the 
Stage-II, Stage-II+SDSS, and Stage-III results, respectively. The gain by a factor of 2 when adding the SDSS data to the Stage-II experiments demonstrates the significant contribution of $\mathrm{BAO}$ and RSD measurements in advancing the cosmological model. The SDSS BAO and RSD data reduce the total volume (within 68\% confidence) of the five-dimensional likelihood surface by a factor of 40 .

The SDSS BAO + RSD measurements have the most significant impact on the precision of $\Omega_{k}, H_{0}$, and $\sum m_{\nu}$. In particular, the combination of Stage-II+SDSS leads to estimates of $H_{0}=67.91 \pm 0.91 \mathrm{~km} \mathrm{~s}^{-1} \mathrm{Mpc}^{-1}$, comparable to the tightest constraints on the local expansion rate presented in Sec. IV B. This result is in disagreement with the combined Cepheid distance ladder and strong lensing time delay results by more than four standard deviations, further reinforcing one of the biggest surprises of the last decade of cosmology results.

\section{B. Beyond dark energy: Cosmology from eBOSS}

The spectroscopic samples from BOSS and eBOSS allow for a diverse array of cosmology studies beyond the cosmic expansion history and growth of structure presented in this work. These data have already been used to advance models for the summed neutrino mass and inflation. In addition, new techniques have been developed to use combinations of tracers or new tracers for direct measurements of BAO and RSD.

These data have been used to place constraints on neutrino masses and inflation parameters through measurements of the one-dimensional flux power spectrum of the Ly $\alpha$ forest $[227,228]$. When combining the recent eBOSS measurement with $\mathrm{CMB}$ and $\mathrm{BAO}$ measurements, the sum of the neutrino masses is constrained with a 95\% upper limit $\sum m_{\nu}<0.09 \mathrm{eV}$ [229]. These same Ly $\alpha$ forest power spectrum measurements present evidence for a departure from a constant value for the power-law index of the primordial power spectrum. The model that best describes the Ly $\alpha$ and Planck data has a running that is nonzero at more than $95 \%$ confidence, $\alpha_{s} \equiv d n_{s} / d \ln k=-0.010 \pm 0.004$.

The eBOSS data have been used to further explore inflationary models through tests for primordial nonGaussianities of the local form, $f_{\mathrm{NL}}$. Recent measurements of the power spectrum in eBOSS quasars offer measurements of $f_{\mathrm{NL}}$ that are independent of the current Planck bispectrum limits. The measurements find $-51<f_{\mathrm{NL}}<21$ at $95 \%$ confidence [230] and indicate that the full eBOSS dataset could reach $\sigma\left(f_{\mathrm{NL}}\right)<10$ using a full range of scales and a larger redshift range.

The five-year eBOSS sample also provides an area that is sampled simultaneously with LRGs, ELGs, and quasars. The overlap in redshifts between samples enables techniques to combine multiple tracers and reduce the effects of sample variance [231,232]. Projections for $f_{\mathrm{NL}}$ and RSD from eBOSS following the multitracer technique are found in the work by Zhao et al. [233]. In an effort to understand the joint clustering across multiple tracers, Alam et al. [234] detect one-halo conformity between the eBOSS LRG and ELG samples at a significance of more than three standard deviations. The result presents the challenges of predicting multitracer clustering at high precision beyond what is possible with the basic halo model. The first eBOSS multitracer cosmology study is associated with this final eBOSS release $[79,235]$. In the configurationspace study, they find an improvement in the RSD measurement precision of approximately $12 \%$ over using the LRG samples presented in this work.

The eBOSS data have inspired several other advanced techniques in cosmology. Tentative BAO measurements have been made at $z<1$ using the cross-correlation between the MgII forest and galaxy and quasar tracers [236]; between the CIV forest and quasars at $z>2$ [237]; and, finally, in the cross-correlation between spectroscopic quasars and high-redshift galaxies [238] selected from the DESI Legacy Imaging Surveys [239]. Voids in the clustering of galaxies and quasars have long been explored as a means to constrain growth of structure and the distanceredshift relation through the AP effect. The first void detections in eBOSS are presented using DR14 LRGs and quasars [240]. This analysis has been extended to the eBOSS DR16, including the ELG sample for the first time in eBOSS and finding a linear redshift-space distortion parameter [241] $\beta^{\mathrm{LRG}}(z=0.74)=0.415 \pm 0.087$, $\beta^{\mathrm{ELG}}(z=0.85)=0.665 \pm 0.125$, and $\beta^{\mathrm{QSO}}(z=1.48)=$ $0.313 \pm 0.134$, consistent with other measurements from eBOSS DR16 using conventional clustering techniques presented in this paper. Ravoux et al. [242] recently developed a parallel technique for void finding at higher redshifts. They derived a three-dimensional map of largescale matter fluctuations from a region that was densely sampled with $\operatorname{Ly} \alpha$ forest quasars. Covering a volume of $0.94 h^{-3} \mathrm{Gpc}^{3}$ with a resolution of $13 h^{-1} \mathrm{Mpc}$, they identify voids and protocluster candidates in the cosmic web.

Finally, the eBOSS data have enabled new techniques for controlling and assessing the selection function for tracers of large-scale structure. Among those new techniques are those results associated with the release of this paper, such as forward modeling of the selection function from imaging surveys [243], new models for fiber collisions [244], and $\mathrm{N}$-body mock catalogs for characterization of the ELG sample [82].

Having recently completed installation and commissioning, the Dark Energy Spectroscopic Instrument (DESI) $[245,246]$ will obtain a sample of nearly 50 million galaxies and quasars spanning the redshift range $0<z<3.5$. The techniques developed in eBOSS to use the one-dimensional Ly $\alpha$ forest flux power spectrum, large-scale clustering, multitracer clustering, void detection, and new models for the selection function and halo occupation statistics will be incorporated into the future DESI studies. This next 
generation of the analyses developed within eBOSS will be an integral part of the final cosmology results at the completion of DESI.

\section{Beyond cosmology: Astrophysics results and potential studies with the eBOSS spectra}

The final eBOSS data sample found in the SDSS Sixteenth Data Release [247] is the result of nearly two decades of development in the spectral data reduction and redshift classification software pipelines. These catalogs of more than four million spectra and their classifications have been extremely well vetted and are ripe for further study. These data have already been used to explore a range of astrophysical processes beyond the cosmology that inspired the program, with continued potential for studies in galaxy evolution, lensing and absorption systems, and quasar physics.

The high-redshift ELG sample is unique within the four generations of SDSS and allows systematic studies of the internal dynamics, composition, and environment of these star-forming galaxies. An example of potential for spectroscopic studies in this large data sample is found in one of the earliest results from eBOSS. Zhu et al. [248] constructed a composite spectrum based on 8,620 galaxies over the redshift interval $0.6<z<1.2$. This composite spectrum reveals blueshifted lines, indicating outflows driven by star formation. This high signal-to-noise spectrum, along with smaller aperture emission line measurements from Hubble Space Telescope and quasar absorption line observations, can all be explained by a self-consistent outflow model. The ELG spectra of roughly 180,000 galaxies were further investigated to constrain the massmetallicity relation at high redshift [249]. The results indicate that the $0.6<z<1.05$ ELGs follow the fundamental metallicity relation that is observed in the local universe. The local environment of the eBOSS galaxies can also be studied through the illumination of the circumgalactic medium. In a study of SDSS quasar spectra, the absorption due to MgII and FeII in intervening LRGs and ELGs was explored over impact parameters ranging from $10 \mathrm{kpc}$ to $1 \mathrm{Mpc}$ [250]. The metal absorption strengths were stronger along the minor axis of the galaxies due to outflowing gas and followed a steeper profile for ELGs than for LRGs, indicating more recent enrichment of the circumgalactic medium due to star formation.

The eBOSS spectra have also been used to identify previously unknown superpositions of multiple galaxy spectra. In a search for serendipitous emission lines in the spectra of BOSS and eBOSS galaxy targets, Talbot et al. [251] were able to identify 1551 strong galaxy-galaxy gravitational lens candidates. The full catalog of these lens candidates is being released as a value-added catalog to enable future studies [252]. Such a large sample can be used to study the demographics of background source galaxies, for advanced modeling of the dark matter structure of lens galaxies with a diverse sample, and for calibrating searches for lens galaxies with ground-based imaging programs.

Finally, eBOSS has unique spectroscopic programs in quasar astrophysics. Three dedicated programs were coordinated with eBOSS to take advantage of the potential for studies in quasar astrophysics:

(i) The Time Domain Spectroscopic Survey (TDSS) $[253,254]$ characterized the spectra of variable stars and quasars identified in photometric imaging.

(ii) The Spectroscopic Identification of eROSITA Sources (SPIDERS) [255,256] observed cluster galaxies and active galactic nuclei detected in the ROSAT All-Sky Survey [257,258] and with XMMNewton [259] observations.

(iii) The SDSS-RM program monitored a sample of 849 quasars at more than 70 epochs over five years. The data enable the measurement of more black hole masses, over a larger range of redshift, than any previous reverberation mapping program [260].

Between the clustering quasar sample [142] and the three quasar programs, quasar targets comprised the majority of all eBOSS spectra. Reverberation mapping studies have measured lags of broad lines relative to the continuum for $\mathrm{H} \alpha$ (17 quasars) [261], $\mathrm{H} \beta$ (442 quasars) [261], MgII (57 quasars) [262], and CIV (52 quasars, in the redshift range $1.4<z<2.8$ ) [263]. Arguably the biggest surprise in quasar astrophysics from SDSS was changing-look quasars that change from broad line quasars with strong continua to narrow line systems with weak continua over the course of a few years [264-266]. This phenomenon had not previously been seen for luminous active galactic nuclei.

Quasar astrophysics is just one of the topics that motivates the next generation of the Sloan Digital Sky Survey, SDSS-V [66]. SDSS-V will provide single-object spectra of more than six million sources across the whole sky with the BOSS spectrographs and the infrared APOGEE spectrographs $[267,268]$. In addition, SDSS-V will perform spatially resolved spectroscopy in the Milky Way and nearby galaxies using new optical spectrographs on several small telescopes. The SDSS-V program will produce the world's premier sample of spectra for studies of Milky Way assembly history, origin of the chemical elements, mapping the local interstellar medium, and time-domain spectroscopy. Scheduled for observations over 2020-2025, the five year program will multiply the science returns from space-based missions such as Gaia and eROSITA [269] while setting the stage for spectroscopy coordinated with imaging from the Vera Rubin Observatory [270].

\section{ACKNOWLEDGMENTS}

This paper represents an effort by both the SDSS-III and SDSS-IV collaborations. Funding for SDSS-III was provided by the Alfred P. Sloan Foundation, the Participating 
Institutions, the National Science Foundation, and the U.S. Department of Energy Office of Science. Funding for the Sloan Digital Sky Survey IV has been provided by the Alfred P. Sloan Foundation, the U.S. Department of Energy Office of Science, and the Participating Institutions. SDSS-IV acknowledges support and resources from the Center for High-Performance Computing at the University of Utah. The SDSS website is www.sdss.org. SDSS-IV is managed by the Astrophysical Research Consortium for the Participating Institutions of the SDSS Collaboration including the Brazilian Participation Group, the Carnegie Institution for Science, Carnegie Mellon University, the Chilean Participation Group, the French Participation Group, Harvard-Smithsonian Center for Astrophysics, Instituto de Astrofísica de Canarias, the Johns Hopkins University, Kavli Institute for the Physics and Mathematics of the Universe (IPMU)/University of Tokyo, Lawrence Berkeley National Laboratory, Leibniz Institut für Astrophysik Potsdam (AIP), Max-Planck-Institut für Astronomie (MPIA Heidelberg), Max-Planck-Institut für Astrophysik (MPA Garching), Max-Planck-Institut für Extraterrestrische Physik (MPE), National Astronomical Observatory of China, New Mexico State University, New York University, University of Notre Dame, Observatário Nacional/MCTI, The Ohio State University, Pennsylvania State University, Shanghai Astronomical Observatory, United Kingdom Participation Group, Universidad Nacional Autónoma de México, University of Arizona, University of Colorado Boulder, University of Portsmouth, University of Utah, University of Virginia, University of Washington, University of Wisconsin, Vanderbilt University, and Yale University.

\section{APPENDIX A: BAO AND RSD MEASUREMENTS AND SYSTEMATIC ERRORS}

In galaxy redshift surveys, BAO and RSD are usually measured through two-point clustering statistics. To calculate the two-point clustering statistics, we convert galaxy redshifts and angular positions into comoving coordinates using a fiducial cosmological model, denoted by a superscript "fid." For a pair of galaxies at effective redshift $z_{\text {eff }}$, with a small separation, the comoving transverse and line-of-sight separations depend on the comoving angular diameter distance $D_{M}\left(z_{\text {eff }}\right)$ and the Hubble distance $D_{H}\left(z_{\mathrm{eff}}\right)=c / H\left(z_{\mathrm{eff}}\right)$, respectively. Conversion from radial comoving distance, $D_{C}$, to $D_{M}$ depends on the cosmological model (see Sec. II). Limiting ourselves to the $\Lambda \mathrm{CDM}$ model, and working in units of $h^{-1} \mathrm{Mpc}$, this conversion depends solely on the value of $\Omega_{m}^{\text {fid }}$. Counts of galaxy pairs, in the form of the correlation function or power spectrum, are then fitted with a fixed model in which the BAO feature is located at $r_{d}^{\text {fid }}$. Although not necessary for the methodology we adopt, we use the same model for computing $r_{d}^{\text {fid }}$ as we do for the conversion of measured coordinates to comoving coordinates.

For the SDSS BAO measurements, we parametrize the position of the $\mathrm{BAO}$ feature using a dimensionless dilation parameter in the transverse direction $\left(\alpha_{\perp}\right)$ and in the radial $\left(\alpha_{\|}\right)$direction. The best-fit values and covariance between these parameters are calculated by fitting the template spectrum to the observed BAO positions in the monopole, quadrupole, and hexadecapole moments of the two-point statistics. Information other than the BAO peak position is removed from the fit by marginalizing over a set of simultaneously fitted parameters that model the shape of the multipole moments.

If the true BAO peak is located at $r_{d}$, which can be different from $r_{d}^{\text {fid }}$, then both $\alpha_{\perp}$ and $\alpha_{\|}$will be shifted by $r_{d}^{\text {fid }} / r_{d}$. The location of the radial BAO peak in the data depends on $D_{H}\left(z_{\text {eff }}\right) / D_{H}^{\text {fid }}\left(z_{\text {eff }}\right)$, while the location of the angular BAO feature depends on $D_{M}\left(z_{\text {eff }}\right) / D_{M}^{\mathrm{fid}}\left(z_{\mathrm{eff}}\right)$. Combining these, we have

$$
\begin{aligned}
& \alpha_{\|}=\frac{D_{H}\left(z_{\mathrm{eff}}\right) / r_{d}}{D_{H}^{\mathrm{fid}}\left(z_{\mathrm{eff}}\right) / r_{d}^{\mathrm{fid}}}, \\
& \alpha_{\perp}=\frac{D_{M}\left(z_{\mathrm{eff}}\right) / r_{d}}{D_{M}^{\mathrm{fid}}\left(z_{\mathrm{eff}}\right) / r_{d}^{\mathrm{fid}}} .
\end{aligned}
$$

We see that $\alpha_{\perp}$ and $\alpha_{\|}$combine information about the model and distance-redshift relationship in a way that is perfectly degenerate. To demonstrate this, we now consider the dependence of the fit on $h / h^{\text {fid }}$.

Working in units of $h^{-1} \mathrm{Mpc}$ and assuming that the fiducial and true physical densities match so $r_{d}=r_{d}^{\text {fid }}$ in units of Mpc, the ratio $h / h^{\text {fid }}$ enters in the shift of the model to $h^{-1} \mathrm{Mpc}$, and hence via $r_{d} / r_{d}^{\text {fid }}$. The ratios $D_{M}^{\mathrm{fid}}\left(z_{\mathrm{eff}}\right) / D_{M}\left(z_{\mathrm{eff}}\right)$ and $D_{H}^{\mathrm{fid}}\left(z_{\mathrm{eff}}\right) / D_{H}\left(z_{\mathrm{eff}}\right)$ are independent of $h$ and $h^{\text {fid }}$ for fixed $\Omega_{m}$. Suppose instead that we had worked in units of Mpc and measured the two-point functions and model in these units. Then, we would have to specify $h^{\text {fid }}$ before calculating the two-point measurements, and so the ratio $h / h^{\mathrm{fid}}$ would enter into the calculation of $D_{M}\left(z_{\text {eff }}\right) / D_{M}^{\mathrm{fid}}\left(z_{\mathrm{eff}}\right)$ and $D_{H}\left(z_{\mathrm{eff}}\right) / D_{H}^{\mathrm{fid}}\left(z_{\mathrm{eff}}\right)$. For models where $r_{d}=r_{d}^{\mathrm{fid}}$, there is no $h$ dependence in the theoretical BAO positions.

Note that the above thought experiment shows that we should always work in the same basis when calculating the components of both $\alpha_{\perp}$ and $\alpha_{\|}$. That is, we should not calculate $r_{d} / r_{d}^{\mathrm{fid}}$ in $\mathrm{Mpc}$ and $D_{M}^{\mathrm{fid}}\left(z_{\mathrm{eff}}\right) / D_{M}\left(z_{\mathrm{eff}}\right)$ in $h^{-1} \mathrm{Mpc}$, for example, which would then ignore the $h / h^{\text {fid }}$ dependence of the fit.

Another way to see this is that the dimensionless quantities $r_{d} / D_{M}\left(z_{\text {eff }}\right)$ and $r_{d} / D_{H}\left(z_{\text {eff }}\right)$ correspond to the size of the BAO feature in observed quantities, namely, angular separations measured in radians and radial separations measured in redshift differences. As long as we 
operate in units that make these ratios truly dimensionless (i.e., without residual dependence on $h$ ), we are performing a correct compression of the available information.

We present our baseline results as $D_{M}\left(z_{\text {eff }}\right) / r_{d}$ and $D_{H}\left(z_{\text {eff }}\right) / r_{d}$ to reflect what is measured in the data. There is no dependence on fiducial values, thus removing potential for ambiguity and the exact values assumed in our fiducial model.

To measure RSD, we fit a template power spectrum or correlation function decomposed into multipoles. We allow the template to be shifted in scale by the dimensionless parameters $\alpha_{\perp}$ and $\alpha_{\|}$and normalized using the parameters $b \sigma_{8}$ and $f \sigma_{8}$. Use of a template spectrum means that our measurements necessarily depend on the shape of this template and on the fiducial cosmology used to create it. As $\sigma_{8}$ is defined as the rms fluctuations on comoving scales of $8 h^{-1}$ Mpc (i.e., not the gauge-independent angular separations and redshift differences), we also need to consider the model dependency of the scale on which the normalization parameters are measured.

In the analysis procedure in SDSS clustering studies, we normalize the template to a predicted $\sigma_{8}$ and find the shifts in scale and normalization required to fit the data. One complication is whether the template is shifted in scale by the dilation parameters before or after the normalization of the model is fixed. Shifting the template before measuring the normalization is equivalent to fixing the scale on which we measure $f \sigma_{8}$ and $b \sigma_{8}$ in the observed two-point clustering, as determined by the fiducial cosmology in units of $h^{-1} \mathrm{Mpc}$. For data at $z=0$, this would result in no cosmological dependence in the scale chosen. However, for measurements at higher redshifts such as those from eBOSS, we have a dependence on the fiducial value of $\Omega_{m}$ used to calculate the distance-redshift relationship.

If, instead, we do not shift the template before fixing the normalization of the model, then we fix the scale in the units of the template. The scale from the template can be different from that preferred by the data, potentially bringing in a further dependence on $h / h^{\text {fid }}$ and changing the degeneracies with other cosmological parameters. In general, we find a larger systematic error contribution for our measurements in this case due to an increased dependence on the fiducial cosmology.

We interpret the dilation parameters as measured from template fits in the same way as those with the above BAO measurements, assuming that the model dependence arises only through $r_{d}$ and not through other scales in the theoretical model. While the BAO scale provides most of the dilation constraint, it is possible that some component arises from other features, and therefore this should be considered an approximation.

For our RSD measurements made using the BOSS and eBOSS galaxy samples, we have found that rescaling the template before fixing the normalization of the model

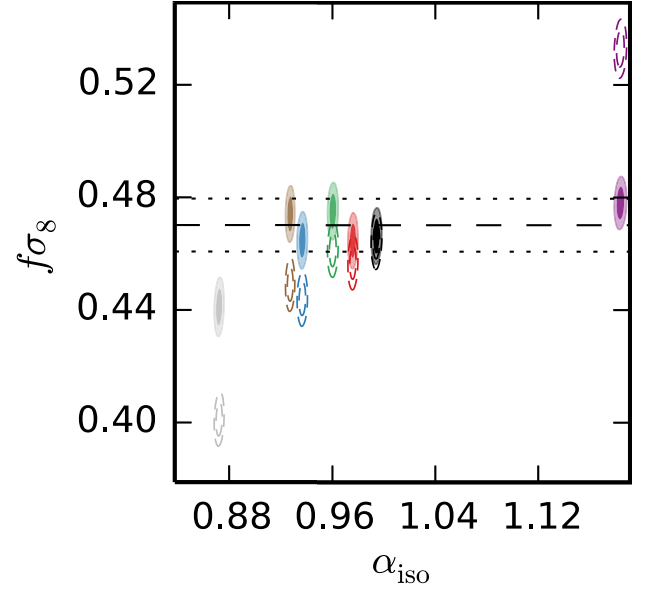

FIG. 15. Dependence of the measured values of $f \sigma_{8}$ on $\alpha_{\text {iso }}=\left(\alpha_{\perp}^{2} \alpha_{\|}\right)^{1 / 3}$, which gives the offset between the template and the true cosmology, calculated from the set of NSERIES mocks matching the BOSS CMASS NGC LRG sample at an effective redshift $z_{\text {eff }}=0.56$. Filled ellipses give results fixing the scale at which $f \sigma_{8}$ is measured after rescaling the template allowing for the shift in the best-fit $\alpha_{\text {iso }}$; the empty ellipses show results where the template is not rescaled. The colors of the ellipses separate fits where different template models were used when analyzing the mocks. Only a weak dependence is seen when $f \sigma_{8}$ is constructed from the rescaled $\sigma_{8}$, significantly smaller than the statistical errors on the measured values. Dotted lines mark a $2 \%$ deviation with respect to the expected $f \sigma_{8}$ value. Further details of these fits are in Ref. [69].

significantly reduces the dependence on fiducial cosmology, and hence the required systematic error.

The $\sigma_{8}$-based normalization measurements we present and analyze retain a dependence on the fiducial $\Omega_{m}$ that sets the scale on which they are measured, and on the shape of the template, which links the scales on which $f \sigma_{8}$ and $b \sigma_{8}$ are defined to those constrained by the data. These dependencies are illustrated in Fig. 7 of Ref. [68] by comparing recovered measurements (from the LRG correlation function) with mock catalogs where the fiducial cosmology assumed is different from the true cosmology. For the power spectrum as measured from the LRGs, we show how measurements of $f \sigma_{8}$ depend on $\alpha_{\text {iso }}=\left(\alpha_{\perp}^{2} \alpha_{\|}\right)^{1 / 3}$ in Fig. 15 . From these tests, we see only a weak dependence on the fiducial assumptions, given our baseline procedure. The scatter in the measurements is included in our assumed systematic error.

For our MGS and eBOSS quasar measurements, the contribution to the systematic error from the fiducial cosmology is significantly reduced compared to other contributions to the systematic error, and we instead adopt the slightly simpler procedure where we do not rescale the template before normalization. This results in slightly larger estimates of the systematic error induced by the analysis method. More details about the systematic errors and the dependencies on the fiducial assumptions in the 
TABLE IX. Cosmological parameters and priors.

\begin{tabular}{lcccc}
\hline \hline Parameterization & Parameter $^{\mathrm{a}}$ & Baseline $^{\mathrm{b}}$ & Prior & Analysis \\
\hline CMB & $\omega_{c}$ & 0.12 & $(0.001,0.99)$ & whenever CMB likelihood is included, \\
& $\omega_{b}$ & 0.0221 & $(0.005,0.1)$ & as in Secs. IV, V, VI. \\
& $100 \theta_{\mathrm{MC}}$ & 1.0411 & $(0.5,10)$ & $\ldots$ \\
& $\ln \left(10^{10} A_{s}\right)$ & 3.05 & $(1.61,3.91)$ & $\ldots$ \\
& $n_{s}$ & 0.96 & $(0.8,1.2)$ & $\ldots$ \\
\multirow{2}{*}{ Background } & $N_{\text {eff }}$ & 0.06 & $(0.01,0.8)$ & $\ldots$ \\
& $\Omega_{m}$ & $\ldots .046$ & $(0.1,0.9)$ & Sec. IVA (SN only, BAO only) \\
Extended & $\Omega_{b}$ & $\ldots$ & $(0.001,0.3)$ & Sec. IVA (BAO only) \\
& $H_{0}\left(\mathrm{~km} \mathrm{~s}^{-1} \mathrm{Mpc}^{-1}\right)$ & $\ldots$ & $(20,100)$ & Sec. IV B 2 \\
& $\Omega_{k}$ & 0.0 & $(-0.8,0.8)$ & Secs. IVA 1, VI B \\
& $w\left(w_{0}\right)$ & -1 & $(-3,1)$ & Secs. IVA 2, VI B \\
& $w_{a}$ & 0 & $(-3,0.75)$ & Sec. VI.B \\
\hline \hline
\end{tabular}

${ }^{\mathrm{a}}$ For each parameter, in parentheses is the range of the flat prior.

${ }^{\mathrm{b}}$ The baseline value of a parameter is the one adopted whenever the parameter is fixed in an analysis.

analysis method can be found in the papers describing the individual measurements.

We have shown that the approximations we made to compress BOSS and eBOSS data into combinations of parameters $\left(\alpha_{\perp}, \alpha_{\|}, f \sigma_{8}\right.$, and $\left.b \sigma_{8}\right)$ do not significantly impact the interpretation of the growth measurements. The compression therefore has minimal impact on our conclusions in testing cosmological models after allowing for appropriate systematic errors. This analysis of the clustering might not be the best approach for future surveys including DESI and Euclid. In order not to compromise the precision available with these future data, it may be best to directly fit models to the two-point multipoles without an intermediate data compression step.

\section{APPENDIX B: MODEL PARAMETRIZATION AND PARAMETER PRIORS}

For cosmological models considered in this study, it is possible to adopt a single parametrization. However, it is convenient to choose a parametrization depending on the investigation, thus allowing different priors depending on the constraints provided by the probes being used. We employ two sets of parametrization. The definitions of parameters can be found in Table I.

The first parametrization is labeled as the $\mathrm{CMB}$ parametrization, which is used whenever CMB likelihoods are included in the analysis. It follows the natural degeneracy direction of the $\mathrm{CMB}$ constraints. The basic parameters in this parametrization include $\omega_{c}, \omega_{b}, \theta_{\mathrm{MC}}, A_{s}$, $n_{s}, \tau$, and $N_{\text {eff }}$.

The second parametrization is labeled as the background parametrization. This parametrization is used for chains without a CMB likelihood, such as in studying SN-only or BAO-only constraints. The basic parameters include $\Omega_{m}$, $H_{0}, \Omega_{b}$ with the latter two used for BAO constraints.

In addition to the basic parameters, we also introduce extended parameters for testing extensions to the $\Lambda \mathrm{CDM}$ model, which include $\Omega_{k}, w$ or $w_{0}, w_{a}$, and $\Sigma m_{\nu}$.

In Table IX, we list the parameters for each parametrization, along with the priors assigned in the analyses and the sections/subsections where the priors are adopted. The baseline value of a parameter refers to the value used whenever the parameter is fixed in an analysis. In addition to the flat priors on parameters that are being varied in the analysis, the $\mathrm{CMB}$ parametrization includes a prior on $H_{0}$ of $20 \mathrm{kms}^{-1} \mathrm{Mpc}^{-1}<H_{0}<100 \mathrm{~km} \mathrm{~s}^{-1} \mathrm{Mpc}^{-1}$. We also applied a Gaussian prior on $n_{s}$ of $n_{s}=0.96 \pm 0.02$ and a $\mathrm{BBN}$-inspired prior of $\omega_{b}=0.0222 \pm 0.0005$ in all runs that include growth information but without $\mathrm{CMB}$ data, i.e., in Figs. 9 (left) and 11. In Sec. IV B 2, for $H_{0}$ constraints without $\mathrm{CMB}$ data, a $\mathrm{BBN}$-motivated Gaussian prior is used for $\omega_{b}(0.02235 \pm 0.00037)$.
[1] A. G. Riess, A. V. Filippenko, P. Challis, A. Clocchiatti, A. Diercks, P. M. Garnavich, R. L. Gilliland, C. J. Hogan, S. Jha, R. P. Kirshner, B. Leibundgut, M. M. Phillips,
D. Reiss, B. P. Schmidt, R. A. Schommer, R. C. Smith, J. Spyromilio, C. Stubbs, N. B. Suntzeff, and J. Tonry, Astron. J. 116, 1009 (1998). 
[2] S. Perlmutter et al., Astrophys. J. 517, 565 (1999).

[3] A. D. Miller, R. Caldwell, M. J. Devlin, W. B. Dorwart, T. Herbig, M. R. Nolta, L. A. Page, J. Puchalla, E. Torbet, and H. T. Tran, Astrophys. J. Lett. 524, L1 (1999).

[4] P. de Bernardis et al., Nature (London) 404, 955 (2000).

[5] A. Balbi, P. Ade, J. Bock, J. Borrill, A. Boscaleri, P. De Bernardis, P. G. Ferreira, S. Hanany, V. Hristov, A. H. Jaffe, A. T. Lee, S. Oh, E. Pascale, B. Rabii, P. L. Richards, G. F. Smoot, R. Stompor, C. D. Winant, and J. H. P. Wu, Astrophys. J. Lett. 545, L1 (2000).

[6] A. H. Jaffe et al., Phys. Rev. Lett. 86, 3475 (2001).

[7] C. B. Netterfield et al., Astrophys. J. 571, 604 (2002).

[8] S. Hanany, P. Ade, A. Balbi, J. Bock, J. Borrill, A. Boscaleri, P. de Bernardis, P. G. Ferreira, V. V. Hristov, A. H. Jaffe, A. E. Lange, A. T. Lee, P. D. Mauskopf, C. B. Netterfield, S. Oh, E. Pascale, B. Rabii, P. L. Richards, G. F. Smoot, R. Stompor, C. D. Winant, and J. H. P. Wu, Astrophys. J. Lett. 545, L5 (2000).

[9] G. Efstathiou, W. J. Sutherland, and S. J. Maddox, Nature (London) 348, 705 (1990).

[10] J. P. Ostriker and P. J. Steinhardt, Nature (London) 377, 600 (1995).

[11] M. Colless et al., Mon. Not. R. Astron. Soc. 328, 1039 (2001).

[12] W. J. Percival et al., Mon. Not. R. Astron. Soc. 327, 1297 (2001).

[13] W. J. Percival et al., Mon. Not. R. Astron. Soc. 337, 1068 (2002).

[14] J. W. Fowler, M. D. Niemack, S. R. Dicker, A. M. Aboobaker, P. A. R. Ade, E. S. Battistelli, M. J. Devlin, R. P. Fisher, M. Halpern, P. C. Hargrave, A. D. Hincks, M. Kaul, J. Klein, J. M. Lau, M. Limon, T. A. Marriage, P. D. Mauskopf, L. Page, S. T. Staggs, D. S. Swetz, E. R. Switzer, R. J. Thornton, and C. E. Tucker, Appl. Opt. 46, 3444 (2007).

[15] J. E. Carlstrom et al., Publ. Astron. Soc. Pac. 123, 568 (2011).

[16] C. L. Bennett, D. Larson, J. L. Weiland, N. Jarosik, G. Hinshaw, N. Odegard, K. M. Smith, R. S. Hill, B. Gold, M. Halpern, E. Komatsu, M. R. Nolta, L. Page, D. N. Spergel, E. Wollack, J. Dunkley, A. Kogut, M. Limon, S. S. Meyer, G. S. Tucker, and E. L. Wright, Astrophys. J. Suppl. 208, 20 (2013).

[17] S. Jha et al., Astron. J. 131, 527 (2006).

[18] A. G. Riess, L.-G. Strolger, S. Casertano, H. C. Ferguson, B. Mobasher, B. Gold, P. J. Challis, A. V. Filippenko, S. Jha, W. Li, J. Tonry, R. Foley, R. P. Kirshner, M. Dickinson, E. MacDonald, D. Eisenstein, M. Livio, J. Younger, C. Xu, T. Dahlén, and D. Stern, Astrophys. J. 659, 98 (2007).

[19] J. A. Frieman et al., Astron. J. 135, 338 (2008).

[20] K. S. Dawson et al., Astron. J. 138, 1271 (2009).

[21] M. Hicken, W. M. Wood-Vasey, S. Blondin, P. Challis, S. Jha, P. L. Kelly, A. Rest, and R. P. Kirshner, Astrophys. J. 700, 1097 (2009).

[22] C. Contreras, M. Hamuy, M. M. Phillips, G. Folatelli, N. B. Suntzeff, S. E. Persson, M. Stritzinger, L. Boldt, S. González, W. Krzeminski, N. Morrell, M. Roth, F. Salgado, M. José Maureira, C. R. Burns, W. L. Freedman, B. F. Madore, D. Murphy, P. Wyatt, W. Li, and A. V. Filippenko, Astron. J. 139, 519 (2010).
[23] J. Guy et al., Astron. Astrophys. 523, A7 (2010).

[24] A. Conley et al., Astrophys. J. Suppl. 192, 1 (2011).

[25] M. Sullivan et al., Astrophys. J. 737, 102 (2011).

[26] A. G. Riess, L. Macri, S. Casertano, M. Sosey, H. Lampeitl, H. C. Ferguson, A. V. Filippenko, S. W. Jha, W. Li, R. Chornock, and D. Sarkar, Astrophys. J. 699, 539 (2009).

[27] W. L. Freedman, B. F. Madore, V. Scowcroft, C. Burns, A. Monson, S. E. Persson, M. Seibert, and J. Rigby, Astrophys. J. 758, 24 (2012).

[28] D. G. York et al., Astron. J. 120, 1579 (2000).

[29] D. J. Eisenstein et al., Astrophys. J. 633, 560 (2005).

[30] S. Cole et al., Mon. Not. R. Astron. Soc. 362, 505 (2005).

[31] W. J. Percival, S. Cole, D. J. Eisenstein, R. C. Nichol, J. A. Peacock, A. C. Pope, and A. S. Szalay, Mon. Not. R. Astron. Soc. 381, 1053 (2007).

[32] G. Hinshaw, J. L. Weiland, R. S. Hill, N. Odegard, D. Larson, C. L. Bennett, J. Dunkley, B. Gold, M. R. Greason, N. Jarosik, E. Komatsu, M. R. Nolta, L. Page, D. N. Spergel, E. Wollack, M. Halpern, A. Kogut, M. Limon, S. S. Meyer, G. S. Tucker, and E. L. Wright, Astrophys. J. Suppl. 180, 225 (2009).

[33] M. Kowalski et al., Astrophys. J. 686, 749 (2008).

[34] E. Komatsu, J. Dunkley, M. R. Nolta, C. L. Bennett, B. Gold, G. Hinshaw, N. Jarosik, D. Larson, M. Limon, L. Page, D. N. Spergel, M. Halpern, R. S. Hill, A. Kogut, S. S. Meyer, G. S. Tucker, J. L. Weiland, E. Wollack, and E. L. Wright, Astrophys. J. Suppl. 180, 330 (2009).

[35] P. A. R. Ade et al. (Planck Collaboration), Astron. Astrophys. 571, A17 (2014).

[36] N. Aghanim et al. (Planck Collaboration), Astron. Astrophys. 641, A8 (2020).

[37] C. Heymans, L. Van Waerbeke, L. Miller, T. Erben, H. Hildebrandt, H. Hoekstra, T. D. Kitching, Y. Mellier, P. Simon, C. Bonnett, J. Coupon, L. Fu, J. Harnois Déraps, M. J. Hudson, M. Kilbinger, K. Kuijken, B. Rowe, T. Schrabback, E. Semboloni, E. van Uitert, S. Vafaei, and M. Velander, Mon. Not. R. Astron. Soc. 427, 146 (2012).

[38] H. Hildebrandt et al., Astron. Astrophys. 633, A69 (2020).

[39] B. Joachimi et al., Astron. Astrophys. 646, A129 (2021).

[40] J. Zuntz et al., Mon. Not. R. Astron. Soc. 481, 1149 (2018).

[41] T. M. C. Abbott et al. (Dark Energy Survey Collaboration), Phys. Rev. D 98, 043526 (2018).

[42] R. Mandelbaum et al., Publ. Astron. Soc. Jpn. 70, S25 (2018).

[43] C. Hikage et al., Publ. Astron. Soc. Jpn. 71, 43 (2019).

[44] S. Joudaki, H. Hildebrandt, D. Traykova, N. E. Chisari, C. Heymans, A. Kannawadi, K. Kuijken, A. H. Wright, M. Asgari, T. Erben, H. Hoekstra, B. Joachimi, L. Miller, T. Tröster, and J. L. van den Busch, Astron. Astrophys. 638, L1 (2020).

[45] A. G. Riess, S. Casertano, W. Yuan, L. M. Macri, and D. Scolnic, Astrophys. J. 876, 85 (2019).

[46] N. Aghanim et al. (Planck Collaboration), Astron. Astrophys. 641, A6 (2020).

[47] K. S. Dawson et al., Astron. J. 145, 10 (2013).

[48] D. J. Eisenstein, D. H. Weinberg, E. Agol, H. Aihara, C. Allende Prieto, S. F. Anderson, J. A. Arns, É. Aubourg, S. Bailey, E. Balbinot et al., Astron. J. 142, 72 (2011). 
[49] L. Anderson et al., Mon. Not. R. Astron. Soc. 427, 3435 (2012).

[50] A. G. Riess, L. Macri, S. Casertano, H. Lampeitl, H. C. Ferguson, A. V. Filippenko, S. W. Jha, W. Li, and R. Chornock, Astrophys. J. 730, 119 (2011).

[51] F. Beutler, C. Blake, M. Colless, D. H. Jones, L. StaveleySmith, L. Campbell, Q. Parker, W. Saunders, and F. Watson, Mon. Not. R. Astron. Soc. 416, 3017 (2011).

[52] N. Padmanabhan, X. Xu, D. J. Eisenstein, R. Scalzo, A. J. Cuesta, K. T. Mehta, and E. Kazin, Mon. Not. R. Astron. Soc. 427, 2132 (2012).

[53] C. Blake et al., Mon. Not. R. Astron. Soc. 425, 405 (2012).

[54] G. Hinshaw, D. Larson, E. Komatsu, D. N. Spergel, C. L. Bennett, J. Dunkley, M. R. Nolta, M. Halpern, R. S. Hill, N. Odegard, L. Page, K. M. Smith, J. L. Weiland, B. Gold, N. Jarosik, A. Kogut, M. Limon, S. S. Meyer, G. S. Tucker, E. Wollack, and E. L. Wright, Astrophys. J. Suppl. 208, 19 (2013).

[55] M. Chevallier and D. Polarski, Int. J. Mod. Phys. D 10, 213 (2001).

[56] E. V. Linder, Phys. Rev. Lett. 90, 091301 (2003).

[57] S. Alam et al., Mon. Not. R. Astron. Soc. 470, 2617 (2017).

[58] J. E. Bautista et al., Astron. Astrophys. 603, A12 (2017).

[59] H. du Mas des Bourboux et al., Astron. Astrophys. 608, A130 (2017).

[60] R. Adam, P. A. R. Ade, N. Aghanim, Y. Akrami, M. I. R. Alves, F. Argüeso, M. Arnaud, F. Arroja, M. Ashdown et al. (Planck Collaboration), Astron. Astrophys. 594, A1 (2016).

[61] J. E. Gunn et al., Astron. J. 131, 2332 (2006).

[62] K. S. Dawson et al., Astron. J. 151, 44 (2016).

[63] https://www.sdss.org/surveys/eboss/

[64] M. R. Blanton, M. A. Bershady, B. Abolfathi, F. D. Albareti, C. Allende Prieto, A. Almeida, J. Alonso-García, F. Anders, S. F. Anderson, B. Andrews et al., Astron. J. 154, 28 (2017).

[65] S. A. Smee et al., Astron. J. 146, 32 (2013).

[66] J. A. Kollmeier et al., arXiv:1711.03234.

[67] https://www.sdss.org/science/cosmology-results-fromeboss/

[68] J. E. Bautista et al., Mon. Not. R. Astron. Soc. 500, 736 (2021).

[69] H. Gil-Marín et al., Mon. Not. R. Astron. Soc. 498, 2492 (2020).

[70] A. Raichoor et al., Mon. Not. R. Astron. Soc. 500, 3254 (2021).

[71] A. Tamone et al., Mon. Not. R. Astron. Soc. 499, 5527 (2020).

[72] A. de Mattia et al., Mon. Not. R. Astron. Soc. 501, 5616 (2021).

[73] J. Hou et al., Mon. Not. R. Astron. Soc. 500, 1201 (2021).

[74] R. Neveux, E. Burtin, A. de Mattia, A. Smith, A. J. Ross, J. Hou, J. Bautista, J. Brinkmann, C.-H. Chuang, K. S. Dawson, H. Gil-Marín, B. W. Lyke, A. de la Macorra, H. du Mas des Bourboux, E.-M. Müller, A. D. Myers, J. A. Newman, W. J. Percival, G. Rossi, D. Schneider, M. Vivek, P. Zarrouk, C. Zhao, and G.-B. Zhao, Mon. Not. R. Astron. Soc. 499, 210 (2020).
[75] H. du Mas des Bourboux et al., Astrophys. J. 901, 153 (2020).

[76] A. J. Ross et al., Mon. Not. R. Astron. Soc. 498, 2354 (2020).

[77] B. W. Lyke et al., Astrophys. J. Suppl. 250, 8 (2020).

[78] S. Lin, J. L. Tinker, A. Klypin, F. Prada, M. R. Blanton, J. Comparat, K. S. Dawson, A. de Mattia, H. du Mas des Bourboux, W. J. Percival, A. Raichoor, G. Rossi, A. Smith, and C. Zhao, Mon. Not. R. Astron. Soc. 498, 5251 (2020).

[79] C. Zhao, C.-H. Chuang, J. Bautista, A. de Mattia, A. Raichoor, A. J. Ross, J. Hou, R. Neveux, C. Tao, E. Burtin, K. S. Dawson, S. de la Torre, H. Gil-Marín, J.-P. Kneib, W. J. Percival, G. Rossi, A. Tamone, J. L. Tinker, G.-B. Zhao, S. Alam, and E.-M. Mueller, arXiv:2007.08997.

[80] J. Farr, A. Font-Ribera, H. du Mas des Bourboux, A. Muñoz-Gutiérrez, F. J. Sánchez, A. Pontzen, A.X. González-Morales, D. Alonso, D. Brooks, P. Doel, T. Etourneau, J. Guy, J.-M. Le Goff, A. de la Macorra, N. Palanque-Delabrouille, I. Pérez-Ràfols, J. Rich, A. Slosar, G. Tarle, D. Yutong, and K. Zhang, J. Cosmol. Astropart. Phys. 20 (2020) 068.

[81] S. Alam et al., arXiv:2007.09004.

[82] S. Avila et al., Mon. Not. R. Astron. Soc. 499, 5486 (2020).

[83] G. Rossi, P. D. Choi, J. Moon, J. E. Bautista, H. Gil-Marín, R. Paviot, M. Vargas-Magaña, S. de la Torre, S. Fromenteau, A. J. Ross, S. Ávila, E. Burtin, K. S. Dawson, S. Escoffier, S. Habib, K. Heitmann, J. Hou, E.-M. Mueller, W. J. Percival, A. Smith, C. Zhao, and G.-B. Zhao, Mon. Not. R. Astron. Soc. (2020), https://doi.org/10.1093/ mnras/staa3955.

[84] A. Smith, E. Burtin, J. Hou, R. Neveux, A. J. Ross, S. Alam, J. Brinkmann, K. S. Dawson, S. Habib, K. Heitmann, J.-P. Kneib, B. W. Lyke, H. du Mas des Bourboux, E.-M. Mueller, A. D. Myers, W. J. Percival, G. Rossi, D. P. Schneider, P. Zarrouk, and G.-B. Zhao, Mon. Not. R. Astron. Soc. 499, 269 (2020).

[85] https://www.sdss.org/science/final-bao-and-rsdmeasurements/

[86] M. Vardanyan, R. Trotta, and J. Silk, Mon. Not. R. Astron. Soc. 397, 431 (2009).

[87] K. Akita and M. Yamaguchi, J. Cosmol. Astropart. Phys. 20 (2020) 012.

[88] G. Mangano, G. Miele, S. Pastor, T. Pinto, O. Pisanti, and P. D. Serpico, Nucl. Phys. B729, 221 (2005).

[89] P. de Salas, D. Forero, C. Ternes, M. Trtola, and J. Valle, Phys. Lett. B 782, 633 (2018).

[90] J. Lesgourgues and S. Pastor, Phys. Rep. 429, 307 (2006).

[91] A. Slosar, Phys. Rev. D 73, 123501 (2006).

[92] A. Font-Ribera, P. McDonald, N. Mostek, B. A. Reid, H.-J. Seo, and A. Slosar, J. Cosmol. Astropart. Phys. 14 (2014) 023.

[93] F. de Bernardis, T.D. Kitching, A. Heavens, and A. Melchiorri, Phys. Rev. D 80, 123509 (2009).

[94] R. Jimenez, T. Kitching, C. Peña-Garay, and L. Verde, J. Cosmol. Astropart. Phys. 10 (2010) 035.

[95] L. Wang and P. J. Steinhardt, Astrophys. J. 508, 483 (1998).

[96] E. V. Linder, Phys. Rev. D 72, 043529 (2005).

[97] E. V. Linder and R. N. Cahn, Astropart. Phys. 28, 481 (2007). 
[98] T. M. C. Abbott et al., Phys. Rev. D 99, 123505 (2019).

[99] G.-B. Zhao, L. Pogosian, A. Silvestri, and J. Zylberberg, Phys. Rev. D 79, 083513 (2009).

[100] S. Staggs, J. Dunkley, and L. Page, Rep. Prog. Phys. 81, 044901 (2018).

[101] Y. Akrami et al. (Planck Collaboration), Astron. Astrophys. 641, A1 (2020).

[102] K. Pardo and D. N. Spergel, Phys. Rev. Lett. 125, 211101 (2020).

[103] D. J. Eisenstein, H.-J. Seo, E. Sirko, and D. N. Spergel, Astrophys. J. 664, 675 (2007).

[104] D. H. Weinberg, M. J. Mortonson, D. J. Eisenstein, C. Hirata, A. G. Riess, and E. Rozo, Phys. Rep. 530, 87 (2013).

[105] C. Alcock and B. Paczynski, Nature (London) 281, 358 (1979).

[106] N. Kaiser, Mon. Not. R. Astron. Soc. 227, 1 (1987).

[107] S. Nadathur, W. J. Percival, F. Beutler, and H. A. Winther, Phys. Rev. Lett. 124, 221301 (2020).

[108] W. E. Ballinger, J. A. Peacock, and A. F. Heavens, Mon. Not. R. Astron. Soc. 282, 877 (1996).

[109] B. Jain and U. Seljak, Astrophys. J. 484, 560 (1997).

[110] P. Astier et al., Astron. Astrophys. 447, 31 (2006).

[111] M. Hamuy, M. M. Phillips, N. B. Suntzeff, R. A. Schommer, J. Maza, and R. Aviles, Astron. J. 112, 2391 (1996).

[112] M. M. Phillips, P. Lira, N. B. Suntzeff, R. A. Schommer, M. Hamuy, and J. Maza, Astron. J. 118, 1766 (1999).

[113] M. Betoule et al., Astron. Astrophys. 568, A22 (2014).

[114] D. M. Scolnic et al., Astrophys. J. 859, 101 (2018).

[115] A. Goobar and B. Leibundgut, Annu. Rev. Nucl. Part. Sci. 61, 251 (2011).

[116] B. Yu, R. Z. Knight, B. D. Sherwin, S. Ferraro, L. Knox, and M. Schmittfull, arXiv:1809.02120.

[117] C.-T. Chiang, W. Hu, Y. Li, and M. LoVerde, Phys. Rev. D 97, 123526 (2018).

[118] A. Lewis and S. Bridle, Phys. Rev. D 66, 103511 (2002).

[119] A. Lewis, arXiv:1910.13970.

[120] A. J. Ross, L. Samushia, C. Howlett, W. J. Percival, A. Burden, and M. Manera, Mon. Not. R. Astron. Soc. 449, 835 (2015).

[121] C. Howlett, A. J. Ross, L. Samushia, W. J. Percival, and M. Manera, Mon. Not. R. Astron. Soc. 449, 848 (2015).

[122] T. Okumura et al., Publ. Astron. Soc. Jpn. 68, 38 (2016).

[123] A. Pezzotta et al., Astron. Astrophys. 604, A33 (2017).

[124] F. Beutler, C. Blake, M. Colless, D. H. Jones, L. StaveleySmith, G. B. Poole, L. Campbell, Q. Parker, W. Saunders, and F. Watson, Mon. Not. R. Astron. Soc. 423, 3430 (2012).

[125] P. Carter, F. Beutler, W. J. Percival, C. Blake, J. Koda, and A. J. Ross, Mon. Not. R. Astron. Soc. 481, 2371 (2018).

[126] F. Beutler, C. Blake, J. Koda, F. A. Marín, H.-J. Seo, A. J. Cuesta, and D. P. Schneider, Mon. Not. R. Astron. Soc. 455, 3230 (2016).

[127] K. N. Abazajian, J. K. Adelman-McCarthy, M. A. Agüeros, S. S. Allam, C. Allende Prieto, D. An, K. S. J. Anderson, S. F. Anderson, J. Annis, N. A. Bahcall et al., Astrophys. J. Suppl. 182, 543 (2009).

[128] http://sdss.physics.nyu.edu/vagc/lss.html
[129] B. Reid et al., Mon. Not. R. Astron. Soc. 455, 1553 (2016).

[130] A. J. Ross et al., Mon. Not. R. Astron. Soc. 464, 1168 (2017).

[131] M. Vargas-Magaña, S. Ho, A. J. Cuesta, R. O’Connell, A. J. Ross, D. J. Eisenstein, W. J. Percival, J. N. Grieb, A. G. Sánchez, J. L. Tinker, R. Tojeiro, F. Beutler, C.-H. Chuang, F.-S. Kitaura, F. Prada, S. A. Rodríguez-Torres, G. Rossi, H.-J. Seo, J. R. Brownstein, M. Olmstead, and D. Thomas, Mon. Not. R. Astron. Soc. 477, 1153 (2018).

[132] F. Beutler et al., Mon. Not. R. Astron. Soc. 464, 3409 (2017).

[133] F. Beutler et al., Mon. Not. R. Astron. Soc. 466, 2242 (2017).

[134] S. Satpathy, S. Alam, S. Ho, M. White, N. A. Bahcall, F. Beutler, J. R. Brownstein, C.-H. Chuang, D. J. Eisenstein, J. N. Grieb, F. Kitaura, M. D. Olmstead, W. J. Percival, S. Salazar-Albornoz, A. G. Sánchez, H.-J. Seo, D. Thomas, J. L. Tinker, and R. Tojeiro, Mon. Not. R. Astron. Soc. 469, 1369 (2017).

[135] A. G. Sánchez et al., Mon. Not. R. Astron. Soc. 464, 1640 (2017).

[136] J. N. Grieb et al., Mon. Not. R. Astron. Soc. 467, 2085 (2017).

[137] A. G. Sánchez, J. N. Grieb, S. Salazar-Albornoz, S. Alam, F. Beutler, A. J. Ross, J. R. Brownstein, C.-H. Chuang, A. J. Cuesta, D. J. Eisenstein, F.-S. Kitaura, W. J. Percival, F. Prada, S. Rodríguez-Torres, H.-J. Seo, J. Tinker, R. Tojeiro, M. Vargas-Magaña, J. A. Vazquez, and G.-B. Zhao, Mon. Not. R. Astron. Soc. 464, 1493 (2017).

[138] E. L. Wright et al., Astron. J. 140, 1868 (2010).

[139] A. Prakash et al., Astrophys. J. Suppl. 224, 34 (2016).

[140] B. Flaugher et al., Astron. J. 150, 150 (2015).

[141] A. Raichoor et al., Mon. Not. R. Astron. Soc. 471, 3955 (2017).

[142] A. D. Myers et al., Astrophys. J. Suppl. 221, 27 (2015).

[143] N. P. Ross et al., Astrophys. J. Suppl. 199, 3 (2012).

[144] N. Palanque-Delabrouille, C. Magneville, C. Yèche, I. Pâris, P. Petitjean, E. Burtin, K. Dawson, I. McGreer, A. D. Myers, G. Rossi, D. Schlegel, D. Schneider, A. Streblyanska, and J. Tinker, Astron. Astrophys. 587, A41 (2016).

[145] P. McDonald and J. Miralda-Escudé, Astrophys. J. 518, 24 (1999).

[146] U. Seljak, J. Cosmol. Astropart. Phys. 12 (2012) 004.

[147] https://svn.sdss.org/public/data/eboss/DR16cosmo/tags/ v1_0_0/likelihoods/.

[148] https://github.com/evamariam/CosmoMC_SDSS2020.

[149] P. A. R. Ade, N. Aghanim, M. Arnaud, M. Ashdown, J. Aumont, C. Baccigalupi, M. Baker, A. Balbi, A. J. Banday et al. (Planck Collaboration), Astron. Astrophys. 536, A1 (2011).

[150] N. Aghanim et al. (Planck Collaboration), Astron. Astrophys. 641, A5 (2020).

[151] https://wiki.cosmos.esa.int/planck-legacy-archive/index .php/Cosmological_Parameters with a description of the COSMOMC implementation at https://cosmologist.info/ cosmomc/readme_planck.html

[152] https://lambda.gsfc.nasa.gov/product/map/current/ likelihood_get.cfm 
[153] M. Sako et al., Publ. Astron. Soc. Pac. 130, 064002 (2018).

[154] N. Kaiser, W. Burgett, K. Chambers, L. Denneau, J. Heasley, R. Jedicke, E. Magnier, J. Morgan, P. Onaka, and J. Tonry, in Society of Photo-Optical Instrumentation Engineers (SPIE) Conference Series, Society of PhotoOptical Instrumentation Engineers (SPIE) Conference Series (SPIE, 2010), Vol. 7733, https://doi.org/10.1117/ 12.859188 .

[155] A. G. Riess et al., Astron. J. 117, 707 (1999).

[156] G. Folatelli et al., Astron. J. 139, 120 (2010).

[157] M. D. Stritzinger et al., Astron. J. 142, 156 (2011).

[158] N. Suzuki et al., Astrophys. J. 746, 85 (2012).

[159] S. A. Rodney et al., Astron. J. 148, 13 (2014).

[160] O. Graur et al., Astrophys. J. 783, 28 (2014).

[161] A. G. Riess et al., Astrophys. J. 853, 126 (2018).

[162] https://archive.stsci.edu/prepds/ps1cosmo/

[163] M. A. Troxel et al. (DES Collaboration), Phys. Rev. D 98, 043528 (2018).

[164] E. Krause et al., arXiv:1706.09359.

[165] https://github.com/cmbant/CosmoMC/blob/master/batch3/ DES_lensing.ini

[166] https://github.com/cmbant/CosmoMC/blob/master/batch3/ DES.ini

[167] P. A. R. Ade et al. (Planck Collaboration), Astron. Astrophys. 594, A13 (2016).

[168] W. Hu, ASP Conf. Ser. 339, 215 (2005), https://ui.adsabs .harvard.edu/abs/2005ASPC..339..215H/abstract.

[169] A. G. Riess, L. M. Macri, S. L. Hoffmann, D. Scolnic, S. Casertano, A. V. Filippenko, B. E. Tucker, M. J. Reid, D. O. Jones, J. M. Silverman, R. Chornock, P. Challis, W. Yuan, P. J. Brown, and R. J. Foley, Astrophys. J. 826, 56 (2016).

[170] W. L. Freedman, B. F. Madore, T. Hoyt, I. S. Jang, R. Beaton, M. G. Lee, A. Monson, J. Neeley, and J. Rich, Astrophys. J. 891, 57 (2020).

[171] J. D. Neill, M. Seibert, R. B. Tully, H. Courtois, J. G. Sorce, T. H. Jarrett, V. Scowcroft, and F. J. Masci, Astrophys. J.. 792, 129 (2014).

[172] B. P. Abbott, R. Abbott, T. D. Abbott, F. Acernese, K. Ackley, C. Adams, T. Adams, P. Addesso, R. X. Adhikari, V. B. Adya et al., Nature (London) 551, 85 (2017).

[173] K. C. Wong et al., Mon. Not. R. Astron. Soc. 498, 1420 (2020).

[174] R. B. Tully, H. M. Courtois, and J. G. Sorce, Astron. J. 152, 50 (2016).

[175] D. W. Pesce, J. A. Braatz, M. J. Reid, A. G. Riess, D. Scolnic, J. J. Condon, F. Gao, C. Henkel, C. M. V. Impellizzeri, C. Y. Kuo, and K. Y. Lo, Astrophys. J. Lett. 891, L1 (2020).

[176] S. Birrer, T. Treu, C. E. Rusu, V. Bonvin, C. D. Fassnacht, J. H. H. Chan, A. Agnello, A. J. Shajib, G. C.-F. Chen, M. Auger, F. Courbin, S. Hilbert, D. Sluse, S. H. Suyu, K. C. Wong, P. Marshall, B. C. Lemaux, and G. Meylan, Mon. Not. R. Astron. Soc. 484, 4726 (2019).

[177] T. M. Davis, S. R. Hinton, C. Howlett, and J. Calcino, Mon. Not. R. Astron. Soc. 490, 2948 (2019).

[178] S. Dhawan, D. Brout, D. Scolnic, A. Goobar, A. G. Riess, and V. Miranda, Astrophys. J. 894, 54 (2020).

[179] R. I. Anderson, Astron. Astrophys. 631, A165 (2019).
[180] D. K. Hazra, A. Shafieloo, and T. Souradeep, J. Cosmol. Astropart. Phys. 04 (2019) 036.

[181] X. Li and A. Shafieloo, Astrophys. J. Lett. 883, L3 (2019).

[182] G. Alestas, L. Kazantzidis, and L. Perivolaropoulos, Phys. Rev. D 101, 123516 (2020).

[183] E. Di Valentino, A. Melchiorri, O. Mena, and S. Vagnozzi, Phys. Rev. D 101, 063502 (2020).

[184] V. Poulin, T. L. Smith, T. Karwal, and M. Kamionkowski, Phys. Rev. Lett. 122, 221301 (2019).

[185] C.-T. Chiang and A. Slosar, arXiv:1811.03624.

[186] R. Beradze and M. Gogberashvili, arXiv:2001.05874.

[187] S. Vagnozzi, Phys. Rev. D 102, 023518 (2020).

[188] M.-X. Lin, G. Benevento, W. Hu, and M. Raveri, Phys. Rev. D 100, 063542 (2019).

[189] N. Arendse, R. J. Wojtak, A. Agnello, G. C. F. Chen, C. D. Fassnacht, D. Sluse, S. Hilbert, M. Millon, V. Bonvin, K. C. Wong, F. Courbin, S. H. Suyu, S. Birrer, T. Treu, and L. V.E. Koopmans, Astron. Astrophys. 639, A57 (2020).

[190] L. Knox and M. Millea, Phys. Rev. D 101, 043533 (2020).

[191] A. J. Cuesta, L. Verde, A. Riess, and R. Jimenez, Mon. Not. R. Astron. Soc. 448, 3463 (2015).

[192] É. Aubourg et al., Phys. Rev. D 92, 123516 (2015).

[193] D. J. Fixsen, Astrophys. J. 707, 916 (2009).

[194] R. J. Cooke, M. Pettini, and C. C. Steidel, Astrophys. J. 855, 102 (2018).

[195] E. G. Adelberger et al., Rev. Mod. Phys. 83, 195 (2011).

[196] G. E. Addison, D. J. Watts, C. L. Bennett, M. Halpern, G. Hinshaw, and J. L. Weiland, Astrophys. J. 853, 119 (2018).

[197] A. Cuceu, J. Farr, P. Lemos, and A. Font-Ribera, J. Cosmol. Astropart. Phys. 19 (2019) 044.

[198] B. P. Abbott et al. (INTEGRAL Collaboration), Astrophys. J. Lett. 848, L13 (2017).

[199] A. Zucca, L. Pogosian, A. Silvestri, and G. B. Zhao, J. Cosmol. Astropart. Phys. 19 (2019) 001.

[200] https://github.com/sfu-cosmo/MGCAMB

[201] A. Shafieloo, V. Sahni, and A. A. Starobinsky, Phys. Rev. D 86, 103527 (2012).

[202] V. Sahni, A. Shafieloo, and A. A. Starobinsky, Astrophys. J. Lett. 793, L40 (2014).

[203] C. Heymans et al., Astron. Astrophys. 646, A140 (2021).

[204] T. Tröster, A. G. Sánchez, M. Asgari, C. Blake, M. Crocce, C. Heymans, H. Hildebrandt, B. Joachimi, S. Joudaki, A. Kannawadi, C.-A. Lin, and A. Wright, Astron. Astrophys. 633, L10 (2020).

[205] G. d'Amico, J. Gleyzes, N. Kokron, K. Markovic, L. Senatore, P. Zhang, F. Beutler, and H. Gil-Marín, J. Cosmol. Astropart. Phys. 05 (2020) 005.

[206] M. M. Ivanov, M. Simonović, and M. Zaldarriaga, J. Cosmol. Astropart. Phys. 20 (2020) 042.

[207] A. Albrecht, G. Bernstein, R. Cahn, W. L. Freedman, J. Hewitt, W. Hu, J. Huth, M. Kamionkowski, E. W. Kolb, L. Knox, J.C. Mather, S. Staggs, and N. B. Suntzeff, arXiv:astro-ph/0609591.

[208] K. Abe et al. (T2K Collaboration), Phys. Rev. Lett. 112, 061802 (2014).

[209] Y. Abe et al., Phys. Rev. D 86, 052008 (2012).

[210] J. K. Ahn et al., Phys. Rev. Lett. 108, 191802 (2012), arXiv:1204.0626. 
[211] F. P. An, J. Z. Bai, A. B. Balantekin, H. R. Band, D. Beavis, W. Beriguete, M. Bishai, S. Blyth, K. Boddy, R. L. Brown et al., Phys. Rev. Lett. 108, 171803 (2012).

[212] P. Adamson et al., Phys. Rev. Lett. 101, 131802 (2008).

[213] T. Araki et al., Phys. Rev. Lett. 94, 081801 (2005).

[214] Q. R. Ahmad et al., Phys. Rev. Lett. 89, 011301 (2002).

[215] Y. Fukuda et al., Phys. Rev. Lett. 81, 1562 (1998).

[216] I. Esteban, M. C. Gonzalez-Garcia, A. Hernand ez-Cabezudo, M. Maltoni, and T. Schwetz, J. High Energy Phys. 19 (2019) 106.

[217] S. R. Choudhury and S. Hannestad, J. Cosmol. Astropart. Phys. 20 (2020) 037.

[218] G. L. Fogli, E. Lisi, A. Marrone, D. Montanino, A. Palazzo, and A. M. Rotunno, Phys. Rev. D 86, 013012 (2012).

[219] Y.-F. Li, J. Cao, Y. Wang, and L. Zhan, Phys. Rev. D 88, 013008 (2013).

[220] E. K. Akhmedov, S. Razzaque, and A. Y. Smirnov, J. High Energy Phys. 13 (2013) 082.

[221] K. Abe et al., Prog. Theor. Exp. Phys. 2015, $053 \mathrm{C} 02$ (2015).

[222] M. Aker, K. Altenmüller, M. Arenz, M. Babutzka, J. Barrett, S. Bauer, M. Beck, A. Beglarian, J. Behrens, T. Bergmann et al., Phys. Rev. Lett. 123, 221802 (2019).

[223] S. Hannestad, Phys. Rev. Lett. 95, 221301 (2005).

[224] S. Weinberg, Rev. Mod. Phys. 61, 1 (1989).

[225] P. Brax and P. Valageas, Phys. Rev. D 99, 123506 (2019).

[226] W. J. Percival et al., Mon. Not. R. Astron. Soc. 401, 2148 (2010).

[227] N. Palanque-Delabrouille et al., Astron. Astrophys. 559, A85 (2013).

[228] S. Chabanier, N. Palanque-Delabrouille, C. Yèche, J.-M. Le Goff, E. Armengaud, J. Bautista, M. Blomqvist, N. Busca, K. Dawson, T. Etourneau, A. Font-Ribera, Y. Lee, H. du Mas des Bourboux, M. Pieri, J. Rich, G. Rossi, D. Schneider, and A. Slosar, J. Cosmol. Astropart. Phys. 19 (2019) 017.

[229] N. Palanque-Delabrouille, C. Yèche, N. Schöneberg, J. Lesgourgues, M. Walther, S. Chabanier, and E. Armengaud, J. Cosmol. Astropart. Phys. 20 (2020) 038.

[230] E. Castorina, N. Hand, U. Seljak, F. Beutler, C.-H. Chuang, C. Zhao, H. Gil-Marín, W. J. Percival, A. J. Ross, P. D. Choi, K. Dawson, A. de la Macorra, G. Rossi, R. Ruggeri, D. Schneider, and G.-B. Zhao, J. Cosmol. Astropart. Phys. 09 (2019) 010.

[231] P. McDonald and U. Seljak, J. Cosmol. Astropart. Phys. 10 (2009) 007.

[232] U. Seljak, Phys. Rev. Lett. 102, 021302 (2009).

[233] G.-B. Zhao et al., Mon. Not. R. Astron. Soc. 457, 2377 (2016).

[234] S. Alam, J. A. Peacock, K. Kraljic, A. J. Ross, and J. Comparat, Mon. Not. R. Astron. Soc. 497, 581 (2020).

[235] Y. Wang et al., Mon. Not. R. Astron. Soc. 498, 3470 (2020).

[236] H. du Mas des Bourboux, K. S. Dawson, N. G. Busca, M. Blomqvist, V. de Sainte Agathe, C. Balland, J. E. Bautista, J. Guy, V. Kamble, A. D. Myers, I. Pérez-Ràfols, M. M. Pieri, J. Rich, D. P. Schneider, and A. Slosar, Astrophys. J. 878, 47 (2019).
[237] M. Blomqvist, H. du Mas des Bourboux, N. G. Busca, V. de Sainte Agathe, J. Rich, C. Balland, J. E. Bautista, K. Dawson, A. Font-Ribera, J. Guy, J.-M. Le Goff, N. Palanque-Delabrouille, W. J. Percival, I. Pérez-Ràfols, M. M. Pieri, D. P. Schneider, A. Slosar, and C. Yèche, Astron. Astrophys. 629, A86 (2019).

[238] P. Zarrouk, M. Rezaie, A. Raichoor, A. J. Ross, S. Alam, R. Blum, D. Brookes, C.-H. Chuang, S. Cole, K. S. Dawson, D. J. Eisenstein, R. Kehoe, M. Landriau, J. Moustakas, A. D. Myers, P. Norberg, W. J. Percival, F. Prada, M. Schubnell, H.-J. Seo, G. Tarlé, and C. Zhao, arXiv:2009.02308.

[239] A. Dey et al., Astron. J. 157, 168 (2019).

[240] A. J. Hawken, M. Aubert, A. Pisani, M.-C. Cousinou, S. Escoffier, S. Nadathur, G. Rossi, and D. P. Schneider, J. Cosmol. Astropart. Phys. 20 (2020) 012.

[241] M. Aubert, M.-C. Cousinou, S. Escoffier, A. J. Hawken, S. Nadathur, S. Alam, J. Bautista, E. Burtin, A. de Mattia, H. Gil-Marín, J. Hou, E. Jullo, R. Neveux, G. Rossi, A. Smith, A. Tamone, and M. Vargas Magaña, arXiv:2007.09013.

[242] C. Ravoux, E. Armengaud, M. Walther, T. Etourneau, D. Pomarède, N. Palanque-Delabrouille, C. Yèche, J. Bautista, H. du Mas des Bourboux, S. Chabanier, K. Dawson, J. M. Le Goff, B. Lyke, A. D. Myers, P. Petitjean, M. M. Pieri, J. Rich, G. Rossi, and D.P. Schneider, J. Cosmol. Astropart. Phys. 20 (2020) 010.

[243] H. Kong, K. J. Burleigh, A. Ross, J. Moustakas, C.-H. Chuang, J. Comparat, A. de Mattia, H. du Mas des Bourboux, K. Honscheid, S. Lin, A. Raichoor, G. Rossi, and C. Zhao, Mon. Not. R. Astron. Soc. 499, 3943 (2020).

[244] F. G. Mohammad et al., Mon. Not. R. Astron. Soc. 498, 128 (2020).

[245] A. Aghamousa, J. Aguilar, S. Ahlen, S. Alam, L. E. Allen, C. Allende Prieto, J. Annis, S. Bailey, C. Balland et al. (DESI Collaboration), arXiv:1611.00036.

[246] A. Aghamousa, J. Aguilar, S. Ahlen, S. Alam, L. E. Allen, C. Allende Prieto, J. Annis, S. Bailey, C. Balland et al., arXiv:1611.00037.

[247] R. Ahumada et al., Astrophys. J. Suppl. 249, 3 (2020).

[248] G. B. Zhu, J. Comparat, J.-P. Kneib, T. Delubac, A. Raichoor, K. S. Dawson, J. Newman, C. Yèche, X. Zhou, and D. P. Schneider, Astrophys. J. 815, 48 (2015).

[249] C. Huang, H. Zou, X. Kong, J. Comparat, Z. Lin, Y. Gao, Z. Liang, T. Delubac, A. Raichoor, J.-P. Kneib, D. P. Schneider, X. Zhou, Q. Yuan, and M. A. Bershady, Astrophys. J. 886, 31 (2019).

[250] T.-W. Lan and H. Mo, Astrophys. J. 866, 36 (2018).

[251] M. S. Talbot, J. R. Brownstein, K. S. Dawson, J.-P. Kneib, and J. Bautista, arXiv:2007.09006.

[252] https://data.sdss.org/sas/dr16/eboss/spectro/lensing/silo

[253] E. Morganson et al., Astrophys. J. 806, 244 (2015).

[254] C. L. MacLeod et al., Astron. J. 155, 6 (2018).

[255] N. Clerc, A. Merloni, Y. Y. Zhang, A. Finoguenov, T. Dwelly, K. Nandra, C. Collins, K. Dawson, J. P. Kneib, E. Rozo, E. Rykoff, T. Sadibekova, J. Brownstein, Y. T. Lin, J. Ridl, M. Salvato, A. Schwope, M. Steinmetz, H. J. Seo, and J. Tinker, Mon. Not. R. Astron. Soc. 463, 4490 (2016).

[256] T. Dwelly, M. Salvato, A. Merloni, M. Brusa, J. Buchner, S. F. Anderson, T. Boller, W. N. Brand t, T. Budavári, N. Clerc, D. Coffey, A. Del Moro, A. Georgakakis, 
P. J. Green, C. Jin, M. L. Menzel, A. D. Myers, K. Nandra, R. C. Nichol, J. Ridl, A. D. Schwope, and T. Simm, Mon. Not. R. Astron. Soc. 469, 1065 (2017).

[257] W. Voges, B. Aschenbach, T. Boller, H. Bräuninger, U. Briel, W. Burkert, K. Dennerl, J. Englhauser, R. Gruber, F. Haberl, G. Hartner, G. Hasinger, M. Kürster, E. Pfeffermann, W. Pietsch, P. Predehl, C. Rosso, J. H. M. M. Schmitt, J. Trümper, and H. U. Zimmermann, Astron. Astrophys. 349, 389 (1999), https://ui.adsabs .harvard.edu/abs/1999A\%26A...349..389V/abstract.

[258] W. Voges, B. Aschenbach, T. Boller, H. Brauninger, U. Briel, W. Burkert, K. Dennerl, J. Englhauser, R. Gruber, F. Haberl, G. Hartner, G. Hasinger, E. Pfeffermann, W. Pietsch, P. Predehl, J. Schmitt, J. Trumper, and U. Zimmermann, VizieR Online Data Catalog, IX/29 (2000), https://ui.adsabs.harvard.edu/abs/2000yCat.9029....0V/ abstract.

[259] F. Jansen, D. Lumb, B. Altieri, J. Clavel, M. Ehle, C. Erd, C. Gabriel, M. Guainazzi, P. Gondoin, R. Much, R. Munoz, M. Santos, N. Schartel, D. Texier, and G. Vacanti, Astron. Astrophys. 365, L1 (2001).

[260] Y. Shen et al., Astrophys. J. Suppl. 216, 4 (2015).

[261] C. J. Grier et al., Astrophys. J. 851, 21 (2017).
[262] Y. Homayouni et al., Astrophys. J. 901, 55 (2020).

[263] C. J. Grier et al., Astrophys. J. 887, 38 (2019).

[264] S. M. LaMassa, S. Cales, E. C. Moran, A. D. Myers, G. T. Richards, M. Eracleous, T. M. Heckman, L. Gallo, and C. M. Urry, Astrophys. J. 800, 144 (2015).

[265] C. L. MacLeod, N. P. Ross, A. Lawrence, M. Goad, K. Horne, W. Burgett, K. C. Chambers, H. Flewelling, K. Hodapp, N. Kaiser, E. Magnier, R. Wainscoat, and C. Waters, Mon. Not. R. Astron. Soc. 457, 389 (2016).

[266] J. Dexter, S. Xin, Y. Shen, C. J. Grier, T. Liu, S. Gezari, I. D. McGreer, W. N. Brand t, P. B. Hall, K. Horne, T. Simm, A. Merloni, P. J. Green, M. Vivek, J. R. Trump, Y. Homayouni, B. M. Peterson, D. P. Schneider, K. Kinemuchi, K. Pan, and D. Bizyaev, Astrophys. J. 885, 44 (2019).

[267] S. R. Majewski et al., Astron. J. 154, 94 (2017).

[268] J. C. Wilson et al., Publ. Astron. Soc. Pac. 131, 055001 (2019).

[269] A. Merloni et al. t. German eROSITA Consortium, arXiv:1209.3114.

[270] C. W. Stubbs, D. Sweeney, J. A. Tyson (LSST Collaboration), Astrophys. J. 873111 (2019). 